\author{
Universidade de Brasília \\ Instituto de Ciências Exatas \\ Departamento de Matemática
}

\title{
Soluções blow-up para equações elípticas com peso singular ou expoente variável
}

\author{
por \\ Luryane Ferreira de Souza
}

Brasília

2015 
Universidade de Brasília

Instituto de Ciências Exatas

Departamento de Matemática

\title{
Soluções blow-up para equações elípticas com peso singular ou expoente variável
}

\author{
por \\ Luryane Ferreira de Souza *
}

Dissertação apresentada ao Departamento de Matemática da Universidade de Brasília, como parte dos requisitos para obtenção do grau de

\section{MESTRE EM MATEMÁTICA}

Brasília, 27 de fevereiro de 2015

Comissão Examinadora:

Dr. Jiazheng Zhou - UnB - Orientador

Dr. Ricardo Ruviaro - UnB - Examinador

Dr. Jefferson Abrantes dos Santos - UFCG - Examinador

*O autor foi bolsista do $\mathrm{CNPq}$ durante a elaboração deste trabalho. 
"Tudo é considerado impossivel até acontecer"

Nelson Mandela 


\section{Agradecimentos}

Primeiramente a Deus que me ilumina todos os dias e é de onde tiro toda coragem e determinação para seguir em frente e não desistir.

A meu pai, Nélio, por todo carinho, amizade, amor, e que durante o mestrado me ajudou incondicionalmente nos dias mais difíceis principalmente nos problemas de saúde. Você é o pilar da nossa família obrigada por ser meu porto seguro.

A minha mãe, Rita, que batalhou muito junto com meu pai para dar a melhor educação e as melhores oportunidades para mim e minhas irmãs. E que infelizmente não viveu para ver essa conquista. Só estou aqui hoje concluindo esse sonho porque você me inspira todos os dias.

As minhas irmãs Lydiane e Yanara pela amizade e alegria que trazem a minha vida, sempre com palavras de incentivo e tranquilizadoras.

Aos meus familiares e amigos, em especial aos que foram mais presentes nesse período meu avô Viterbo, Tio Clésio, Cleonice, Ranivar, Dayane, Rubs, Ana, Bruna, André, Jamer, Ilton, Júlia, Amparo, e a todos tios e tias, primos e primas, amigos e amigas que não citei aqui mas que sempre torceram por mim.

Ao meu orientador Zhou, primeiramente por aceitar me orientar, e pela atenção, paciência e dedicação sempre disposto a ajudar, muito obrigada.

Aos professores da banca Ricardo Ruviaro e Jefferson Abrantes dos Santos por terem aceito o convite e pelas correções e sugestões.

Aos professores e funcionários do departamento pela ajuda e dedicação.

Ao $\mathrm{CNPq}$ pelo apoio financeiro.

Enfim a todos que contribuiram para a realização desse sonho.

Valeu galera! 


\section{Resumo}

Nesse trabalho consideramos o problema

$$
\left\{\begin{array}{llc}
\triangle_{p} u=a(x) u^{q(x)} & \text { em } & \Omega \\
u=+\infty & \text { na } & \partial \Omega
\end{array}\right.
$$

onde $\Omega \subset \mathbb{R}^{n}$ é um domínio limitado ou $\Omega=\mathbb{R}^{n}, p>1$.

Vamos estudar a existência de solução para o problema (1) em dois casos:

1. $\Omega \neq \mathbb{R}^{n}, q(x)=q>p-1$ e $a(x)$ é uma função não negativa, que pode ser singular na $\partial \Omega$.

2. $\Omega=\mathbb{R}^{n}$, para $n \geq 3, p=2, a(x)=1$ e $q$ é uma função Holder contínua, $q(x) \geq 1$ para $\|x\| \leq R$ e $0<q(x) \leq 1$ para $\|x\| \geq R$, onde $R \geq 0$ é uma constante.

Além disso, estudamos a unicidade e comportamento na $\partial \Omega$ para a solução do caso 1 .

Palavras - Chaves: Blow-up sub e supersolução, princípio da comparação, assíntotas, expoente variável. 


\section{Abstract}

In this work we consider the problem

$$
\left\{\begin{array}{llc}
\triangle_{p} u=a(x) u^{q(x)} & \text { em } & \Omega \\
u=+\infty & \text { na } & \partial \Omega
\end{array}\right.
$$

where $\Omega \subset \mathbb{R}^{n}$ is a bounded domain or $\Omega=\mathbb{R}^{n}, p>1$.

We will study existence of solution for problem (2) in two cases:

1. $\Omega \neq \mathbb{R}^{n}, q(x)=q>p-1$ and $a(x)$ is a nonnegative function, wich can be singular on $\partial \Omega$.

2. $\Omega=\mathbb{R}^{n}, n \geq 3, p=2, a(x)=1$ and $q$ is Holder continuous function, $q(x) \geq 1$ for $\|x\| \leq R$ and $0<q(x) \leq 1$ for $\|x\| \geq R$, where $R \geq 0$ is a constant.

Moreover, we study uniqueness and behavior on $\partial \Omega$ for solution of the first case.

Key - Words : Blow-up subsolution and supersolution, comparison principle, asymptotes, variable exponent. 


\section{Sumário}

Introdução 1

1 Resultados Auxiliares 4

1.1 Desigualdade de Gronwall . . . . . . . . . . . . . . . . . 4

1.2 Supersolução e Subsolução . . . . . . . . . . . . . . . . . 5

1.3 Princípio da comparação . . . . . . . . . . . . . . . . . 6

1.4 Teorema de Poincaré-Bendixson . . . . . . . . . . . . . . 7

1.5 Desigualdade de Harnack . . . . . . . . . . . . . . . . . . . 8

1.6 Princípio do máximo forte . . . . . . . . . . . . . . 9

1.7 Função distância . . . . . . . . . . . . . . . . . . . . 9 9

2 Problema com p-Laplaciano e peso singular $\quad 10$

2.1 Resultados Preliminares . . . . . . . . . . . . . . . 11

2.2 Prova da Existência . . . . . . . . . . . . . . . . . . . . . 41

2.3 Estimativa $(2.3)$ do Teorema $(2.1) \ldots \ldots \ldots \ldots \ldots$. . . . . . . . . . . . . . . . . . . . 47

2.4 Unicidade do Teorema $(2.1) \ldots \ldots \ldots \ldots$. . . . . . . . . . . 51

3 Soluções Inteiras para Problemas Elípticos não Lineares com Expoentes Variáveis

3.1 Resultados Preliminares . . . . . . . . . . . . . . . . . 54

3.2 Demonstração do teorema $(3.2 .1)$ e $(3.2 .2) \ldots \ldots \ldots$. . . . . . . 67

A A função $f(u)=u^{q}(x)$ satisfaz a condição de Keller-Osserman $\quad 83$

B Existência de uma solução $u(r)$ que satisfaz (3.3) 85

$\begin{array}{ll}\text { C Prova da Desigualdade (3.5) } & 87\end{array}$

$\begin{array}{ll}\text { D Majorando Integral } & 88\end{array}$

$\begin{array}{lll}\text { E } & \text { Propriedades das funções } g_{*} \text { e } g^{*} & 89\end{array}$

$\begin{array}{lll}\text { F A integral em (3.17) é finita } & 91\end{array}$

$\begin{array}{ll}\text { Anexo } 1 & 92\end{array}$ 
Anexo 2

Bibliografia 


\section{Introdução}

Nesse trabalho vamos estudar existência e unicidade de solução para o problema

$$
\begin{cases}\triangle_{p} u=a(x) u^{q(x)} & x \in \Omega \\ u(x) \rightarrow+\infty & x \in \partial \Omega\end{cases}
$$

onde $p>1$, a é uma função contínua não negativa e singular na fronteira de $\Omega$ e $q: \mathbb{R}^{n} \rightarrow$ $[0, \infty)$, o subconjunto $\Omega$ pode ser um domínio limitado suave de $\mathbb{R}^{n}$, com $u(x) \rightarrow+\infty$ quando $d(x, \partial \Omega) \rightarrow 0$ ou $\Omega=\mathbb{R}^{n}$ então $u(x) \rightarrow+\infty$ quando $|x| \rightarrow+\infty$.

Quando $q(x)=q>p-1, p>1$ e $\Omega \subset \mathbb{R}^{n}$ é limitado, temos que o problema (1) se transforma em

$$
\begin{cases}\triangle_{p} u=a(x) u^{q} & x \in \Omega \\ u \rightarrow+\infty & x \in \partial \Omega,\end{cases}
$$

( ver [5]). Alguns trabalhos, como [2], [13], [20] já provaram existência de solução para problemas parecidos com (2). Em [2] foi provado existência e unicidade de solução para o problema

$$
\begin{cases}\triangle_{p} u=f(u) & x \in \Omega \\ u \rightarrow+\infty & x \in \partial \Omega\end{cases}
$$

onde $f \in C([0, \infty)$ e $f$ é uma função não linear. Já em [13]

$$
\begin{cases}\triangle_{p} u=H(x, u) & x \in \Omega \\ u \rightarrow+\infty & x \in \partial \Omega\end{cases}
$$

onde $H(x, u)=a(x) f(u)$, foi mostrado a existência de solução blow-up para $a(x)$ limitada. Quando $a(x)$ não é limitada, $q>1$, temos, para o caso $p=2$, que existe uma solução para o problema

$$
\begin{cases}\triangle u=a(x) u^{q} & x \in \Omega \\ u \rightarrow+\infty & x \in \partial \Omega,\end{cases}
$$

(ver [20]). Outros trabalhos também estudam condições para $a(x)$, para garantir existên- 
cia e unicidade de solução para (5).

Vamos estudar, também, o problema (1) quando $p=2, a(x)=1, \Omega=\mathbb{R}^{n}$ e $q: \mathbb{R}^{n} \rightarrow$ $[0, \infty)$ uma função localmente Holder contínua, com $q(x) \leq 1$ quando $|x| \rightarrow+\infty$. Neste caso o problema (1) é dado por

$$
\left\{\begin{array}{l}
\triangle u=u^{q(x)} \\
u(x) \stackrel{|x| \rightarrow+\infty}{\longrightarrow}+\infty,
\end{array}\right.
$$

como visto em [9]. Para $q$ constante positiva, temos dos Lemas 2 e 3 de [15] que $\triangle u=u^{q}$ tem uma solução quando $q \leq 1$. Já o problema

$$
\left\{\begin{array}{l}
\triangle u=u^{q(x)} \quad x \in \Omega \\
u=+\infty \quad \text { na } \quad x \in \partial \Omega
\end{array}\right.
$$

com $\Omega$ limitado, possui uma solução se $q(x)=q>1$ pelo Teorema 1 em [10].

Esse trabalho foi dividido em três capítulos. No primeiro apresentamos algumas definições e resultados que serão usados nos demais capítulos. No Capítulo 2 estudamos a existência e unicidade de solução para o problema (2), na primeira seção desse capítulo provamos todos os teoremas que serão utilizados para provar nas seções seguintes existência, unicidade e a estimatima para o problema:

Teorema 0.1 Sejam $a \in C(\Omega)$ uma função não negativa e $q>p-1$. Tome a $>0$ numa vizinhança da fronteira $\partial \Omega$, e suponha que exista $Q \in C(\bar{\Omega})$, com $\gamma \in C^{\mu}(\bar{\Omega}), 0<\mu \leq 1$, tais que $Q(x)>0$ na $\partial \Omega$ e $\gamma(x)<0$ para $x \in \partial \Omega$ tais que

$$
\lim _{x \rightarrow x_{0}} d(x)^{\gamma(x)} a(x)=Q\left(x_{0}\right)
$$

onde $d(x)=d(x, \partial \Omega)$ e $x_{0} \in \partial \Omega$. O problema (2) admite uma única solução fraca positiva $u \in W_{l o c}^{1, p}(\Omega) \cap C_{l o c}^{1, \eta}(\Omega)$ para algum $\eta \in(0,1)$, que verifica

$$
\lim _{x \rightarrow x_{0}} d(x)^{\alpha(x)} u(x)=\left(\frac{(p-1) \alpha\left(x_{0}\right)^{p-1}\left(\alpha\left(x_{0}\right)+1\right)}{Q\left(x_{0}\right)}\right)^{\frac{1}{q-p+1}}
$$

para todo $x_{0} \in \partial \Omega$, onde $\alpha(x)=\frac{p-\gamma(x)}{q-p+1}$.

Assumindo que $q(x)$ é uma função limitada fora de uma bola de $\mathbb{R}^{n}$ verificando algumas das seguintes condições:

Q1- $q: \mathbb{R}^{n} \rightarrow(0, \infty)$ é localmente Holder contínua

Q2-existe $R \geq 0$ tal que $q(x) \geq 1$ para $|x| \leq R$, e $0<q(x) \leq 1$ para $|x| \geq R$.

Quando $R=0$, a condição Q2 é entendida como $0<q(x) \leq 1 \mathrm{em} \mathbb{R}^{n}$. 
Então, mostraremos no capítulo 3, a existência de solução para o problema (6) a partir dos respectivos teoremas:

Teorema 0.2 Suponha q satisfaz condições $Q \mathbf{1}$ e Q2. Se

$$
\int_{0}^{r} r \exp \left(\lambda r^{2}\right) q_{o s c}(r) d r<\infty
$$

para algum $\lambda$ tal que $2 N \lambda>1$, então o problema (6) admite infinitas soluções.

$\mathrm{E} q_{o s c}(r)=q^{*}(r)-q_{*}(r), q^{*}(r):=\max _{|x|=r} q(x)$ e $q_{*}(r):=\min _{|x|=r} q(x)$.

Para o próximo teorema definimos $\|q\|_{\infty}:=\max \left\{q(x) ; x \in \mathbb{R}^{n}\right\}$, segue

Teorema 0.3 Assuma que $q(x)$ verifica a condição $Q \mathbf{1}$ e suponha que existam constantes $0<R<\sqrt{2^{1-\|q\|_{\infty}}}$ e $0<\beta \leq 1$ tais que $0 \leq q(x) \leq \beta$ para todo $|x| \geq R$. Mais ainda, assuma que $q_{*}(r) \leq 1$ para $r>0$. Se

$$
\int_{1}^{\infty} r v_{\beta}(r) q_{o s c}(r) d r<\infty
$$

onde

$$
v_{\beta}(r):= \begin{cases}r^{\frac{2}{1-\beta}} & \text { se } 0<\beta<1, r \in \mathbb{R} \\ \exp \left(r^{2}\right) & \text { se } \beta=1 e r \in \mathbb{R}\end{cases}
$$

então o problema (6) admite uma solução positiva. 


\section{Capítulo}

\section{Resultados Auxiliares}

Nesse capítulo vamos revisar alguns resultados e definições que serão utilizados em todo o texto. Muitos dos resultados aqui apresentados não serão demonstrados, mas segue a referência onde há a demonstração.

\subsection{Desigualdade de Gronwall}

Teorema 1.1.1 (Desigualdade de Gronwall) Sejam $\alpha: I \rightarrow \mathbb{R}$ e $v: I \rightarrow \mathbb{R}$ funções continuas definidas num intervalo $I=[a, b)$ com $a<b \leq \infty$. Suponha que $v(x) \geq 0$, $x \in I$, e u é uma função contínua que satisfaz

$$
u(t) \leq \alpha(t)+\int_{a}^{t} v(s) u(s) d s \quad t \in I,
$$

então

$$
u(t)=\alpha(t)+\int_{a}^{t} \alpha(s) v(s) e^{\int_{s}^{t} v(r) d r} d s .
$$

A demostração dessa desigualdade pode ser vista no Lema 1.1 de [12].

Teorema 1.1.2 (Desigualdade de Gronwall Generalizada) Sejam $g, f:[0, b] \rightarrow \mathbb{R}$ funções integráveis, não negativas e a $:[0, T] \rightarrow \mathbb{R}$ uma função contínua, não negativa tais que:

$$
g(t) \leq g_{0}+\int_{0}^{t} f(s) d s+\int_{0}^{t} a(s) g(s) d s
$$


onde $g_{0}$ é uma constante não negativa. Então

$$
g(t) \leq\left(g_{0}+\int_{0}^{t} f(s) d s\right) e^{\int_{0}^{t} a(s) d s}
$$

A demostração está apresentada no Teorema 1.15 de [11].

\subsection{Supersolução e Subsolução}

Considere o problema elíptico quasilinear do tipo

$$
\begin{aligned}
-\operatorname{div} A(x, \nabla u)+B(x, u, \nabla u) & =0 \quad x \in \Omega \\
u & =g \quad x \in \partial \Omega
\end{aligned}
$$

onde $\Omega$ é um domínio limitado em $\mathbb{R}^{n}, A$ é uma função vetorial de n-vetores cujas variáveis são $x$ e $\nabla u=\left(\frac{\partial u}{\partial x_{1}}, \ldots, \frac{\partial u}{\partial x_{n}}\right)$, B é uma função escalar dada, de variáveis $x, u$ e $\nabla u$, e $g$ uma função definida na fronteira de $\Omega$. A equação não linear pseudo-Laplaciana, isto é, $A(x, \nabla u)=|\nabla u|^{p-2} \nabla u \mathrm{e}$

$$
-\operatorname{div}\left(|\nabla u|^{p-2} \nabla u\right)+B(x, u, \nabla u)=0 \quad x \in \Omega, \quad p>1
$$

é um caso especial de (1.1).

Definição 1.2.1 Uma função u é dita uma solução de (1.1) em $\Omega$ se $u \in W^{1, p}(\Omega)$, $B(x, u, \nabla u) \in L_{l o c}^{1}(\Omega) e$

$$
\int_{\Omega}\left\{\left(|\nabla u|^{p-2} \nabla u\right) \nabla \varphi+B(x, u, \nabla u) \varphi\right\} d x=0
$$

para todo $\varphi \in C_{0}^{\infty}(\Omega)$.

Definição 1.2.2 Uma função u é dita uma subsolução (ou uma supersolução) de (1.1) em $\Omega$ se $u \in W^{1, p}(\Omega), B(x, u, \nabla u) \in L_{l o c}^{1}(\Omega) e$

$$
\int_{\Omega}\left\{\left(|\nabla u|^{p-2} \nabla u\right) \nabla \varphi+B(x, u, \nabla u) \varphi\right\} d x \leq 0 \quad(\geq 0)
$$

para todo $\varphi \in C_{0}^{\infty}(\Omega) \operatorname{com} \varphi \geq 0$ em $\Omega$.

Definição 1.2.3 Uma função u é dita $W$-subsolução da equação (1.1) em $\Omega$ se u= $\max \left\{u_{i} ; i=1,2, \ldots, m\right\}$ em $\Omega$ para algum $m \in \mathbb{N}$, onde cada $u_{i}$ é uma subsolução de (1.1) em $\Omega$ e $u_{i} \in W^{1, p}(\Omega)$. 
Uma função u é dita ser uma $W$-supersolução da equação (1.1) em $\Omega$ se $u=\min \left\{u_{i} ; i=\right.$ $1,2, \ldots, m\}$ em $\Omega$ para algum $m \in \mathbb{N}$, onde cada $u_{i}$ é uma supersolução de (1.1) em $\Omega$ e $u_{i} \in W^{1, p}(\Omega)$.

O próximo resultado será muito utilizado para garantir existência de solução para problemas do tipo (1.1) e (1.3).

Teorema 1.2.1 Sejam $\varphi_{1}$ e $\varphi_{2}$ respectivamente uma $W$-subsolução e uma $W$-supersolução de (1.1) em $\Omega$ tais que $\varphi_{1} \leq \varphi_{2}$ em $\Omega$ e $\varphi_{1} \leq g \leq \varphi_{2}$ na $\partial \Omega$. Suponha que existe uma constante positiva $c_{1}$ e uma função $f_{3} \in L^{q}(\Omega)$, com $q=\frac{p}{p-1}$ e $0<p<\infty$ tal que

$$
|B(x, t, \xi)| \leq\left|f_{3}(x)\right|+h(|t|)+c_{1}|\xi|^{p-1}
$$

para $x \in \Omega,(t, \xi) \in \mathbb{R} \times \mathbb{R}^{n}$, onde $h: \overline{\mathbb{R}}_{+} \rightarrow \overline{\mathbb{R}}_{+}$é uma função não decrescente tal que $h(|\varphi|) \in L^{q}(\Omega)$ para $\varphi \in L^{p}(\Omega)$. Então o problema (1.1) e (1.2) tem uma solução u tal que $\varphi_{1} \leq u \leq \varphi_{2}$ em $\Omega$.

Este resultado foi provado em [8].

\subsection{Princípio da comparação}

Segundo Tolksdorf (prova em [18]) temos o seguinte resultado, que será muito usado nesse trabalho para comparar duas soluções.

Teorema 1.3.1 Seja $G: \Omega \times \mathbb{R} \rightarrow \mathbb{R}$ continua com $G(x,$.$) não crescente para cada x \in \Omega$. Se $u, w \in W^{1, p}(\Omega)$ satisfazem as inequações

$$
\int_{\Omega}|\nabla u|^{p-2} \nabla u \nabla \varphi \leq \int_{\Omega} G(x, u) \varphi \quad e \quad \int_{\Omega}|\nabla w|^{p-2} \nabla w \nabla \varphi \geq \int_{\Omega} G(x, w) \varphi
$$

para toda função não-negativa $\varphi \in W^{1, p}(\Omega)$, e além disso $u \leq w$ na $\partial \Omega$ então $u \leq w$ em $\Omega$.

O próximo resultado diz respeito a regularidade interior para soluções fracas de equações quase lineares.

Teorema 1.3.2 Suponha $h(x, t)$ é mensurável em $x \in \Omega$ e contínua em $t \in \mathbb{R}$ tal que $|h(x, t)| \leq \Gamma$ em $\Omega \times \mathbb{R}$. Seja $u \in W^{1, p}(\Omega)$ uma solução fraca de $\triangle_{p} u=h(x, u)$. Dado um sub-dominio $D \subset \subset \Omega$, existe $\alpha>0$ e uma constante positiva $C$, dependendo de $n, p, \Gamma,\|u\|_{\infty}$ e D tal que

$$
|\nabla u(x)| \leq C \quad e \quad|\nabla u(x)-\nabla u(y)| \leq C|x-y|^{\alpha}, x, y \in D
$$


A prova desse teorema pode ser encontrada em [3].

\subsection{Teorema de Poincaré-Bendixson}

Seja $A$ um subconjunto aberto do espaço euclidiano $\mathbb{R}^{n}$. A aplicação $X: A \rightarrow \mathbb{R}^{n}$ de classe $C^{k}$ é um campo vetorial de classe $C^{k}$ com $1 \leq k \leq \infty$. Onde $X$ está associado a equação diferencial

$$
x^{\prime}=X(x) .
$$

As aplicações diferenciáveis $\varphi: I \rightarrow A$ (I um intervalo ), que são soluções das equações (1.4), tais que

$$
\frac{d \varphi}{d t}(t)=X(\varphi(t))
$$

para todo $t \in I$, são chamadas tragetórias ou curvas integrais de $X$.

Um ponto $x \in A$ é dito ponto singular de $X$ se $X(x)=0$ e ponto regular de $X$ se $X(x) \neq 0$.

Seja $\varphi(t)=\varphi(t, p)$ a curva integral de $X$ passando pelo ponto $p$ definida no seu intervalo máximo $I_{p}$, onde $I_{p}=\left(\omega_{-}(p), \omega_{+}(p)\right)$. Se $\omega_{+}(p)=\infty$ defini-se o conjunto

$$
\omega(p)=\left\{q \in A ; \exists\left(t_{n}\right) \rightarrow \infty \text { e } \varphi\left(t_{n}\right) \rightarrow q, \text { quando } n \rightarrow \infty\right\} .
$$

Analogamente, se $\omega_{-}(p)=-\infty$, defini-se o conjunto

$$
\alpha(p)=\left\{q \in A ; \exists\left(t_{n}\right) \rightarrow-\infty \text { e } \varphi\left(t_{n}\right) \rightarrow q, \text { quando } n \rightarrow \infty\right\}
$$

Os conjuntos $\omega(p)$ e $\alpha(p)$ são chamados respectivamente de conjunto $\omega$-limite e conjunto $\alpha$-limite de $\mathrm{p}$.

Para enunciarmos o teorema de Poincaré- Bendixson iremos denotar $\gamma_{p}^{+}$como a semiórbita positiva por $p$

$$
\gamma_{p}^{+}=\{\varphi(t, p) ; t \geq 0\}
$$

Teorema 1.4.1 (Poincaré-Bendixon) Seja $\varphi(t)=\varphi(t, p)$ uma curva integral de $X$, defina para todo $t \geq 0$, tal que $\gamma_{p}^{+}$esteja contida num compacto $K \subset A$.

Suponha que o campo $X$ possua um número finito de singularidades em $\omega(p)$. Tem-se as seguintes alternativas:

a) Se $\omega(p)$ contém somente pontos regulares, então $\omega(p)$ é uma órbita periódica;

b) Se $\omega(p)$ contém pontos regulares e singulares, então $\omega(p)$ consiste de um conjunto de 
órbitas, cada uma das quais tende a um desses pontos singulares quando $t \rightarrow \pm \infty$;

c) Se $\omega(p)$ não contém pontos regulares, então $\omega(p)$ é um ponto singular.

A demonstração desse teorema bem como mais resultados desse assunto se encontra no Capítulo VII de [17].

\subsection{Desigualdade de Harnack}

Seja a equação

$$
\operatorname{div} A\left(x, u, u_{x}\right)+B\left(x, u, u_{x}\right)=0
$$

onde $x \in \mathbb{R}^{n}, u_{x}=\left(u_{x_{1}}, \ldots, u_{x_{n}}\right)$, a função $A\left(x, u, u_{x}\right)$ é uma função mensurável em $\mathbb{R}^{n}$ e a função $B\left(x, u, u_{x}\right)$ é uma função mensurável real, ambas definidas em $\Omega \times \mathbb{R} \times \mathbb{R}^{n}$, onde $\Omega$ é um domínio em $\mathbb{R}^{n}$.

Vamos assumir que para todo $M<\infty$ e para todo $(x, u, p) \in \Omega \times(-M, M) \times \mathbb{R}^{n}$, as seguintes condições são satisfeitas

$$
\begin{aligned}
& |A(x, u, p)| \leq a_{0}|p|^{\alpha-1}+\left|a_{1}(x) u\right|^{\alpha-1}+\left(a_{3}(x)\right)^{\alpha-1}, \\
& p A(x, u, p) \geq|p|^{\alpha}-\left|a_{2}(x) u\right|^{\alpha}-\left(a_{4}(x)\right)^{\alpha}, \\
& |B(x, u, p)| \leq b_{0}|p|^{\alpha}+b_{1}(x)|p|^{\alpha-1}+\left(b_{2}(x)\right)^{\alpha}|u|^{\alpha-1}+\left(b_{3}(x)\right)^{\alpha}
\end{aligned}
$$

onde $\alpha>1, a_{0}, b_{0}$ são constantes, $a_{i}(x), b_{i}(x)$ são funções mensuráveis não negativas, $\alpha$, $a_{0}, b_{0}, a_{i}(x), b_{i}(x)$ podem depender de $M$.

Vamos considerar a desigualdade de Harnack para o cubo $K_{x_{0}}(p)$ denotado como um cubo em $\mathbb{R}^{n}$ de lado $p$ e centro $x_{0}$ cujos os lados são paralelos aos eixos coordenados. Em geral escrevemos $K(p)=K_{x_{0}}(p)$ assumindo o centro conhecido.

No teorema que segue, vamos denotar por $C$ uma função $C\left(\lambda_{1}, \ldots, \lambda_{n}\right)$ que depende de $\lambda_{1}, \ldots, \lambda_{n}$ que são diferentes de $u(x)$.

Para a desigualdade de Harnack vamos assumir $a_{3}(x), a_{4}(x), b_{3}(x)=0$.

Teorema 1.5.1 Seja u(x) uma solução fraca de (1.5) em um cubo $K=K(3 \rho) \subset \Omega$ com $0 \leq u<M$ em K. Então

$$
\max _{K(\rho)} u(x) \leq C \min _{K(\rho)} u(x)
$$

onde $C=C\left(\alpha, n, a_{0}, b_{0} M, \mu \rho\right)$ 


\subsection{Princípio do máximo forte}

Definição 1.6.1 Dizemos que um ponto $x_{0} \in \partial \Omega$ satisfaz a condição esférica interior se existe uma bola aberta $B=B_{R}\left(x_{1}\right) \subset \Omega$ tal que $\bar{B} \cap \partial \Omega=\left\{x_{0}\right\}$. E definimos o vetor unitário

$$
v=\frac{x_{1}-x_{0}}{\left|x_{1}-x_{0}\right|}
$$

$v$ é normal a $\partial B$ em $x_{0}$ na direção interior a $\Omega$.

O próximo resultado garante existência de solução positiva ou identicamente nula em $\Omega$

Teorema 1.6.1 Seja $u \in C^{1}(\Omega)$ tal que $\triangle_{p} u \in L_{\text {loc }}^{2}(\Omega), u \geq 0$ em $\Omega, \triangle_{p} u \leq \beta(u)$ em $\Omega$, $\operatorname{com} \beta:[0, \infty) \rightarrow \mathbb{R}$ continua, não decrescente, $\beta(0)=0$ e $\beta(s)=0$ para algum $s>0$ ou $\beta(s)>0$ para todo $s>0$ e $\int_{0}^{1}(\beta(s) s)^{-\frac{1}{p}} d s=+\infty$.

Então se u não é nula em $\Omega$ então u é positiva em $\Omega$.

E se $u \in C^{1}\left(\Omega \cup\left\{x_{0}\right\}\right)$ para $x_{0} \in \partial \Omega$ que satisfaz a condição esférica interior $e$ $u\left(x_{0}\right)=0$ então

$$
\frac{\partial u}{\partial v}\left(x_{0}\right)>0
$$

onde $v$ é um vetor normal interior em $x_{0}$ definido em 1.7 .

A prova desse Teorema está em Vázquez (1984) [19]

\subsection{Função distância}

Seja $\Omega$ um domínio limitado em $\mathbb{R}^{n}$ que possui $\partial \Omega$ não vazia. A função distância $d$ é definida por

$$
d(x)=\operatorname{dist}(x, \partial \Omega) .
$$

Para $\mu>0$, seja $\Gamma_{\mu}=\{x \in \bar{\Omega} \mid d(x)<\mu\}$.

Lema 1.7.1 Seja $\Omega$ um conjunto limmitado e $\partial \Omega \in C^{k}$ para $k \geq 2$. Então existe uma constante positiva $\mu$ dependendo de $\Omega$ tal que $d \in C^{k}\left(\Gamma_{\mu}\right)$.

A prova desse lema está em [7]. 


\section{Capítulo}

2

\section{Problema com p-Laplaciano e peso singular}

Neste capítulo vamos obter uma descrição do conjunto de soluções para o problema elíptico, blow-up, quaselinear

$$
\begin{cases}\triangle_{p} u=a(x) u^{q}(x), & x \in \Omega \\ u \rightarrow+\infty, & x \in \partial \Omega\end{cases}
$$

onde $\Omega$ é um domínio $C^{2}$ limitado de $\mathbb{R}^{n}, \triangle_{p} u=\operatorname{div}\left(|\nabla u|^{p-2} \nabla u\right), p>1$ e $q>p-1$. A função peso, $a(x)$ é contínua em $\Omega$, não negativa. A condição de fronteira $u \rightarrow+\infty$ na $\partial \Omega$ pode ser entendida como $u \rightarrow+\infty$ quando $d(x) \rightarrow 0^{+}$, onde $d(x)$ é a função distância $\operatorname{dist}(x, \partial \Omega)$. Para isso provaremos o seguinte teorema.

Teorema 2.1 Sejam $a \in C(\Omega)$ uma função não negativa com a $>0$ numa vizinhança da fronteira $\partial \Omega$ e $q>p-1$. Suponha que $Q \in C(\bar{\Omega})$ com $Q(x)>0$ na $\partial \Omega$, e $\gamma \in C^{\mu}(\bar{\Omega})$, $0<\mu \leq 1$, e $\gamma<0$ na $\partial \Omega$ verificando:

$$
\lim _{x \rightarrow x_{0}} d(x)^{\gamma(x)} a(x)=Q\left(x_{0}\right)
$$

para todo $x_{0} \in \partial \Omega$. O problema (2.1) admite uma única solução fraca positiva $u \in$ $W_{l o c}^{1, p}(\Omega) \cap C_{l o c}^{1, \eta}(\Omega)$ para algum $\eta \in(0,1)$, verificando:

$$
\lim _{x \rightarrow x_{0}} d(x)^{\alpha(x)} u(x)=\left(\frac{(p-1) \alpha\left(x_{0}\right)^{p-1}\left(\alpha\left(x_{0}\right)+1\right)}{Q\left(x_{0}\right)}\right)^{\frac{1}{q-p+1}}
$$


para todo $x_{0} \in \partial \Omega$, onde $\alpha(x)=\frac{p-\gamma(x)}{q-p+1}$.

Mas antes de provarmos o teorema, vamos estudar alguns resultados que serão importantes na prova desse resultado.

\subsection{Resultados Preliminares}

Nesta seção vamos estudar dois teoremas que vão auxiliar na prova da existência e garantir a estimativa (2.3).

Teorema 2.1.1 Seja $f \in L_{\text {loc }}^{\infty}(\Omega)$ tal que $|f| \leq C_{0} d^{-\beta}$ para $C_{0}>0, \beta \in(0, p)$ e d $(x):=$ $d(x, \partial \Omega)$. Então o problema

$$
\begin{cases}-\triangle_{p} u=f, & x \in \Omega \\ u=0, & x \in \partial \Omega\end{cases}
$$

tem uma única solução fraca $u \in W_{l o c}^{1, p}(\Omega) \cap C_{l o c}^{1, \eta}(\Omega) \cap C(\bar{\Omega})$ para algum $\eta \in(0,1)$. Mais ainda, existe uma constante positiva $C$ que não depende de $f$ tal que

$$
|u| \leq C C_{0}^{\frac{1}{p-1}} d^{\frac{p-\beta}{p-1}}, \quad x \in \Omega .
$$

Prova: a) Suponha $\beta \in(1, p)$. Seja $\phi \in W_{0}^{1, p} \cap C^{1, \eta}(\bar{\Omega})$ para $\eta \in(0,1)$ a única solução do problema

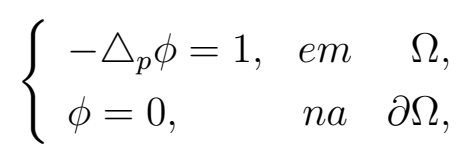

uma vez que $\underline{\phi}=0$ e $\bar{\phi}=1$ são subsolução e supersolução de (2.6) respectivamente (pelo Teorema (1.2.1)).

Pelo princípio do máximo forte, desigualdade (1.8) Teorema (1.6.1), temos

$$
\frac{\partial \phi}{\partial \eta}\left(x_{0}\right)>0 \text { com } x_{0} \in \partial \Omega
$$

onde $\eta$ é vetor normal unitário interno.

AfirmaÇão 1: Existem constantes $\tilde{C}_{1}$ e $\tilde{C}_{2}$ tais que

$$
\tilde{C}_{1} d \leq \phi \leq \tilde{C}_{2} d \quad \text { em } \Omega
$$


De fato, como $\phi \in C^{1}(\Omega)$ e $\frac{\partial \phi}{\partial \eta}>0$ na $\partial \Omega$, seja $\Omega_{\delta_{1}}=\left\{x \in \Omega / d(x)<\delta_{1}\right\}$ tal que

$$
\frac{\partial \phi}{\partial \eta}>0
$$

em $\Omega_{\delta_{1}}$, onde $\eta$ é um vetor normal interior no ponto $x_{0}$.

Do Lema (1.7.1) temos que existe $\delta_{2}>0$ tal que $d \in C^{1}\left(\Omega_{\delta_{2}}\right)$, com $\Omega_{\delta_{2}}=\{x \in$ $\left.\Omega / d(x)<\delta_{2}\right\} \mathrm{e}$

$$
\frac{\partial d}{\partial \eta}>0
$$

em $\Omega_{\delta_{2}}$.

Agora tome $\delta=\min \left\{\delta_{1}, \delta_{2}\right\}$ temos $\frac{\partial \phi}{\partial \eta}>0$ e $\frac{\partial d}{\partial \eta}>0$ em $\Omega_{\delta}$, como $\phi \in C^{1}(\bar{\Omega})$ então existem $a_{1}, b_{1}>0$ tais que

$$
\min _{\overline{\Omega_{\delta}}} \frac{\partial \phi}{\partial \eta}=a_{1} \quad e \quad \max _{\overline{\Omega_{\delta}}} \frac{\partial \phi}{\partial \eta}=b_{1}
$$

Dados $x \in \Omega_{\delta}$ e $\hat{d}: \partial \Omega \longrightarrow \mathbb{R}$ onde $\hat{d}(y)=\|y-x\|$, como $\hat{d}$ é contínua e $\partial \Omega$ é compacta, existe $x_{0} \in \partial \Omega$ tal que $\hat{d}\left(x_{0}\right)$ é mínimo, então $d(x)=\left\|x-x_{0}\right\|=\hat{d}\left(x_{0}\right)$.

Considere $\eta=\frac{x-x_{0}}{\left\|x-x_{0}\right\|}$ vetor normal interno unitário e

$$
\varphi(t)=\phi\left(t x_{0}+(1-t) x\right) \quad t \in[0,1] .
$$

Observe que $\varphi(0)=\phi(x)$ e $\varphi(1)=\phi\left(x_{0}\right)=0$ pois $x_{0} \in \partial \Omega$. Segue do Teorema Fundamental do Cálculo que

$$
\begin{aligned}
\varphi(0)-\varphi(1) & =\int_{1}^{0} \varphi^{\prime}(t) d t=\int_{1}^{0} \nabla \phi\left(t x_{0}+(1-t) x\right) \cdot\left(x_{0}-x\right) d t \\
& =\int_{1}^{0} \nabla \phi\left(t x_{0}+(1-t) x\right) \cdot \frac{\left(x_{0}-x\right)}{\left\|x-x_{0}\right\|}\left\|x-x_{0}\right\| d t \\
& =\int_{0}^{1} \nabla \phi\left(t x_{0}+(1-t) x\right) \cdot \eta\left\|x-x_{0}\right\| d t .
\end{aligned}
$$

Mas, como $\nabla \phi \cdot \eta=\frac{\partial \phi}{\partial \eta}$ temos

$$
\begin{gathered}
\varphi(0)-\varphi(1)=\int_{0}^{1} \frac{\partial \phi}{\partial \eta}\left(t x_{0}+(1-t) x\right)\left\|x-x_{0}\right\| d t \geq a_{1}\left\|x-x_{0}\right\| \\
\Longrightarrow \quad \phi(x) \geq a_{1}\left\|x-x_{0}\right\| .
\end{gathered}
$$




\section{Analogamente}

$$
\begin{gathered}
\varphi(0)-\varphi(1)=\int_{0}^{1} \frac{\partial \phi}{\partial \eta}\left(t x_{0}+(1-t) x\right)\left\|x-x_{0}\right\| d t \leq b_{1}\left\|x-x_{0}\right\| \\
\Longrightarrow \quad \phi(x) \leq b_{1}\left\|x-x_{0}\right\| .
\end{gathered}
$$

Assim temos

$$
a_{1}\left\|x-x_{0}\right\| \leq \phi(x) \leq b_{1}\left\|x-x_{0}\right\| \quad \forall x \in \Omega_{\delta} .
$$

Também existem $a_{2}, b_{2}>0$ tais que

$$
a_{2}=\min _{\overline{\Omega_{\delta}}} \frac{\partial d}{\partial \eta} \quad e \quad b_{2}=\max _{\overline{\Omega_{\delta}}} \frac{\partial d}{\partial \eta} .
$$

\section{Definimos}

$$
\psi(t)=d\left(t x_{0}+(1-t) x\right) \quad t \in[0,1]
$$

e observamos que $\psi(0)=d(x)$ e $\psi(1)=d\left(x_{0}\right)=0$ então

$$
\begin{aligned}
\psi(0)-\psi(1) & =\int_{1}^{0} \psi^{\prime}(t) d t=\int_{1}^{0} \nabla d\left(t x_{0}+(1-t) x\right) \cdot\left(x_{0}-x\right) d t \\
& =\int_{1}^{0} \nabla d\left(t x_{0}+(1-t) x\right) \cdot \frac{\left(x_{0}-x\right)}{\left\|x_{0}-x\right\|}\left\|x_{0}-x\right\| d t \\
& =\int_{0}^{1} \nabla d\left(t x_{0}+(1-t) x\right) \cdot \eta\left\|x_{0}-x\right\| d t .
\end{aligned}
$$

Observando que $\frac{\partial d}{\partial \eta}=\nabla d \cdot \eta$, temos

$$
\begin{aligned}
\psi(0)-\psi(1) & =\int_{0}^{1} \nabla d\left(t x_{0}+(1-t) x\right) \cdot \eta\left\|x_{0}-x\right\| d t \\
& =\int_{0}^{1} \frac{\partial d}{\partial \eta}\left(t x_{0}+(1-t) x\right)\left\|x_{0}-x\right\| d t \\
& \leq b_{2}\left\|x-x_{0}\right\|
\end{aligned}
$$

$\log 0$

$$
d(x) \leq b_{2}\left\|x-x_{0}\right\|
$$


e analogamente

$$
\psi(0)-\psi(1) \geq a_{2}\left\|x-x_{0}\right\| \quad \Rightarrow \quad d(x) \geq a_{2}\left\|x_{0}-x\right\| .
$$

Daí

$$
a_{2}\left\|x_{0}-x\right\| \leq d(x) \leq b_{2}\left\|x_{0}-x\right\| \quad \forall x \in \Omega_{\delta}
$$

Assim, por (2.9) e (2.12) obtemos

$$
\phi(x) \leq b_{1}\left\|x-x_{0}\right\| \leq \frac{b_{1}}{a_{2}} d(x), \quad \forall x \in \Omega_{\delta}
$$

e

$$
\phi(x) \geq a_{1}\left\|x-x_{0}\right\| \geq \frac{a_{1}}{b_{2}} d(x), \quad \forall x \in \Omega_{\delta} .
$$

Portanto

$$
\frac{a_{1}}{b_{2}} d(x) \leq \phi(x) \leq \frac{b_{1}}{a_{2}} d(x) \quad \forall x \in \Omega_{\delta} .
$$

Como $\Omega \backslash \Omega_{\delta}$ é compacto e a função $\phi(x) d^{-1}(x)$ é contínua neste conjunto, temos

$$
c_{1} \leq \phi(x) d^{-1}(x) \leq c_{2} \quad \Rightarrow \quad c_{1} d(x) \leq \phi(x) \leq c_{2} d(x) \text { em } \Omega \backslash \Omega_{\delta} .
$$

De (2.13) e (2.14), mostramos a Afirmação 1.

AfirmaÇÃo 2: Seja $\alpha=\frac{p-\beta}{p-1}, \bar{u}=\phi^{\alpha}$ é uma supersolução de $-\triangle_{p} u=C d^{-\beta}$ para algum $C>0$ que se anula na $\partial \Omega$. Para mostrar a Afirmação 2, devemos mostrar que:

$$
\int_{\Omega}|\nabla \bar{u}|^{p-2} \nabla \bar{u} \nabla \varphi d x \geq \int_{\Omega} C d^{-\beta} \varphi(x) d x
$$

para $\varphi \in W_{0}^{1, p}(\Omega), \varphi \geq 0$ e $\bar{u} \geq 0$ na $\partial \Omega$. Como $\bar{u}=\phi^{\alpha}$, temos $\nabla \bar{u}=\alpha \phi^{\alpha-1} \nabla \phi \mathrm{e}$ $|\nabla \bar{u}|=\alpha \phi^{\alpha-1}|\nabla \phi|$. Segue que

$$
|\nabla \bar{u}|^{p-2} \nabla \bar{u}=\alpha^{p-2} \phi^{(\alpha-1)(p-2)}|\nabla \phi|^{p-2} \alpha \phi^{\alpha-1} \nabla \phi=\alpha^{p-1} \phi^{(\alpha-1)(p-1)}|\nabla \phi|^{p-2} \nabla \phi .
$$

Assim

$$
\begin{aligned}
\int_{\Omega}|\nabla \bar{u}|^{p-2} \nabla \bar{u} \nabla \varphi d x-\int_{\Omega} C d^{-\beta} \varphi(x) d x & =\int_{\Omega} \alpha^{p-1} \phi^{(\alpha-1)(p-1)}|\nabla \phi|^{p-2} \nabla \phi \nabla \varphi d x \\
& -\int_{\Omega} C d^{-\beta} \varphi d x .
\end{aligned}
$$


Por outro lado,

$$
\begin{aligned}
|\nabla \phi|^{p-2} \nabla \phi \nabla\left(\alpha^{p-1} \phi^{(\alpha-1)(p-1)} \varphi\right) & =|\nabla \phi|^{p-2} \nabla \phi \nabla\left(\alpha^{p-1} \phi^{(p-1)(\alpha-1)}\right) \varphi \\
& +\alpha^{p-1} \phi^{(p-1)(\alpha-1)}|\nabla \phi|^{p-2} \nabla \phi \nabla \varphi
\end{aligned}
$$

donde

$$
\begin{aligned}
\alpha^{p-1} \phi^{(\alpha-1)(p-1)}|\nabla \phi|^{p-2} \nabla \phi \nabla \varphi & =|\nabla \phi|^{p-2} \nabla \phi \nabla\left(\alpha^{p-1} \phi^{(p-1)(\alpha-1)} \varphi\right) \\
& -|\nabla \phi|^{p-2} \nabla \phi \nabla\left(\alpha^{p-1} \phi^{(\alpha-1)(p-1)}\right) \varphi
\end{aligned}
$$

De (2.16) e (2.15)

$$
\begin{aligned}
\int_{\Omega}|\nabla \bar{u}|^{p-2} \nabla \bar{u} \nabla \varphi d x & -\int_{\Omega} C d^{-\beta} \varphi d x=\int_{\Omega}|\nabla \phi|^{p-2} \nabla \phi \nabla\left(\alpha^{p-1} \phi^{(p-1)(\alpha-1)} \varphi\right) \\
& -\int_{\Omega}|\nabla \phi|^{p-2} \nabla \phi \nabla\left(\alpha^{p-1} \phi^{(\alpha-1)(p-1)}\right) \varphi-\int_{\Omega} C d^{-\beta} \varphi d x .
\end{aligned}
$$

Uma vez que $\phi$ é solução de (2.6), tem-se

$$
\int_{\Omega}|\nabla \phi|^{p-2} \nabla \phi \nabla \Phi d x=\int_{\Omega} 1 \Phi d x \quad \forall \Phi \in W_{0}^{1, p}(\Omega) .
$$

Em particular para $\Phi=\alpha^{p-1} \phi^{(\alpha-1)(p-1)} \varphi$ temos

$$
\int_{\Omega}|\nabla \phi|^{p-2} \nabla \phi \nabla\left(\alpha^{p-1} \phi^{(\alpha-1)(p-1)} \varphi\right) d x=\int_{\Omega} \alpha^{p-1} \phi^{(\alpha-1)(p-1)} \varphi d x .
$$

Agora observe que

$$
\alpha-1=\frac{p-\beta}{p-1}-1=\frac{p-\beta-p+1}{p-1}=\frac{1-\beta}{p-1}<0
$$


pois $\beta>1$ e $p>1$. Deste fato

$$
\begin{aligned}
|\nabla \phi|^{p-2} \nabla \phi \nabla\left(\alpha^{p-1} \phi^{(\alpha-1)(p-1)}\right) \varphi & =\alpha^{p-1}(\alpha-1)(p-1) \phi^{(\alpha-1)(p-1)-1} \nabla \phi \varphi|\nabla \phi|^{p-2} \nabla \phi \\
& =\alpha^{p-1}(\alpha-1)(p-1) \phi^{(\alpha-1)(p-1)-1} \varphi|\nabla \phi|^{p}<0
\end{aligned}
$$

De (2.18) e (2.19), temos que

$$
\begin{array}{r}
\int_{\Omega}|\nabla \bar{u}|^{p-2} \nabla \bar{u} \nabla \varphi d x-\int_{\Omega} C d^{-\beta} \varphi d x=\int_{\Omega} \alpha^{p-1} \phi^{(\alpha-1)(p-1)} \varphi d x-\int_{\Omega} C d^{-\beta} \varphi d x \\
\quad+\int_{\Omega}(1-\alpha)(p-1) \alpha^{p-1} \phi^{(p-1)(\alpha-1)-1}|\nabla \phi|^{p} \varphi d x \\
=\int_{\Omega}\left[\alpha^{p-1} \phi^{(\alpha-1)(p-1)}-C d^{-\beta}+(1-\alpha)(p-1) \alpha^{p-1} \phi^{(\alpha-1)(p-1)-1}|\nabla \phi|^{p}\right] \varphi d x .
\end{array}
$$

Como $\phi \leq C_{2} d$ e $\alpha-1<0$ temos que

$$
\phi^{(\alpha-1)(p-1)} \geq C_{2}^{(\alpha-1)(p-1)} d^{(\alpha-1)(p-1)}
$$

e

$$
(\alpha-1)(p-1)-1=\alpha p-\alpha-p=p(\alpha-1)-\alpha<0 \text {. }
$$

Assim

$$
\begin{aligned}
\int_{\Omega}|\nabla \bar{u}|^{p-2} \nabla \bar{u} \nabla \varphi & d x-\int_{\Omega} C d^{-\beta} \varphi d x \geq \int_{\Omega}\left[\alpha^{p-1} C_{2}^{(\alpha-1)(p-1)} d^{(\alpha-1)(p-1)}-C d^{-\beta}\right. \\
& \left.+(1-\alpha)(p-1) \alpha^{p-1} C_{2}^{(\alpha-1)(p-1)-1} d^{(\alpha-1)(p-1)-1}|\nabla \phi|^{p}\right] \varphi d x .
\end{aligned}
$$

Uma vez que

$$
(\alpha-1)(p-1)=\left[\frac{p-\beta}{p-1}-1\right](p-1)=\left[\frac{p-\beta-p+1}{p-1}\right](p-1)=1-\beta,
$$

a desigualdade (2.19) fica 


$$
\begin{array}{r}
\int_{\Omega}|\nabla \bar{u}|^{p-2} \nabla \bar{u} \nabla \varphi d x-\int_{\Omega} C d^{-\beta} \varphi d x \geq \int_{\Omega}\left[\alpha^{p-1} C_{2}^{1-\beta} d^{1-\beta}+(\beta-1) \alpha^{p-1}|\nabla \phi|^{p} C_{2}^{-\beta} d^{-\beta}\right. \\
\left.\quad-C d^{-\beta}\right] \varphi d x \\
\int_{\Omega} d^{-\beta}\left[\alpha^{p-1} C_{2}^{1-\beta} d+(\beta-1) \alpha^{p-1}|\nabla \phi|^{p} C_{2}^{-\beta}-C\right] \varphi d x .
\end{array}
$$

AfIRMAÇÃo 3: Deste fato, para mostrarmos a Afirmação 2, basta mostrarmos

$$
\alpha^{p-1} C_{2}^{(1-\beta)} d+(\beta-1) \alpha^{p-1}|\nabla \phi|^{p} C_{2}^{-\beta}-C>0
$$

para um $C>0$ adequado.

Vimos anteriormente que $|\nabla \phi|>0$ em $\Omega \backslash \Omega^{\prime}$ e $d>0$ em $\Omega^{\prime}$. Como $1-\alpha>0, p-1>0$, $\alpha>0$ e $\min _{\Omega^{\prime}} d>0$, temos

$\alpha^{p-1} C_{2}^{1-\beta} d+(\beta-1) \alpha^{p-1}|\nabla \phi|^{p} C_{2}^{-\beta} \geq \alpha^{p-1} C_{2}^{1-\beta} d>\alpha^{p-1} C_{2}^{(1-\beta)} \min _{\Omega^{\prime}} d>0$. em $\Omega^{\prime}$ Agora

$$
\begin{aligned}
\alpha^{p-1} C_{2}^{1-\beta} d+(\beta-1) \alpha^{p-1}|\nabla \phi|^{p} C_{2}^{-\beta} & >(\beta-1) \alpha^{p-1}|\nabla \phi|^{p} C_{2}^{-\beta} \\
& \geq(\beta-1) \alpha^{p-1} C_{2}^{-\beta} \min _{\Omega \backslash \Omega^{\prime}}|\nabla \phi|^{p}>0, \text { em } \Omega \backslash \Omega^{\prime} .
\end{aligned}
$$

Sendo assim, tomando $C:=\min \left\{\alpha^{p-1} C_{2}^{1-\beta} \min _{\Omega^{\prime}} d,(\beta-1) \alpha^{p-1} C_{2}^{-\beta} \min _{\Omega \backslash \Omega^{\prime}}|\nabla \phi|^{p}\right\}>0$, temos finalmente que

$$
\alpha^{p-1} C_{2}^{(1-\beta)} d+(\beta-1) \alpha^{p-1}|\nabla \phi|^{p} C_{2}^{-\beta}-C>0
$$

Logo

$$
-\triangle_{p} \bar{u} \geq C d^{-\beta}
$$

Agora, considere a família de domínios $\Omega_{k}=\left\{x \in \Omega ; d(x)>\frac{1}{k}\right\}$. Tendo em vista que $f \in L^{\infty}\left(\Omega_{k}\right)$ para todo $k \in \mathbb{N}$, o problema

$$
\left\{\begin{array}{l}
-\triangle_{p} u=f \quad x \in \Omega_{k} \\
u=0 \quad x \in \partial \Omega_{k}
\end{array}\right.
$$


possui uma única solução $u_{k} \in W_{0}^{1, p}\left(\Omega_{k}\right) \cap C^{1, \eta}\left(\bar{\Omega}_{k}\right)$. Por hipótese, $|f| \leq C_{0} d^{-\beta} \Rightarrow f \leq$ $C_{0} d^{-\beta}$, assim

$$
-\triangle_{p} u_{k}=f \leq C_{0} d^{-\beta} \leq-\triangle_{p}\left(\left(\frac{C_{0}}{C}\right)^{\frac{1}{p-1}} \bar{u}\right) \text { em } \Omega_{k}
$$

pois

$$
\begin{aligned}
-\triangle_{p}\left(\left(\frac{C_{0}}{C}\right)^{\frac{1}{p-1}} \bar{u}\right) & =-\operatorname{div}\left(\left|\nabla\left[\left(\frac{C_{0}}{C}\right)^{\frac{1}{p-1}} \bar{u}\right]\right|^{p-2} \nabla\left[\left(\frac{C_{0}}{C}\right)^{\frac{1}{p-1}} \bar{u}\right]\right) \\
& =-\frac{C_{0}}{C} \operatorname{div}\left(|\nabla \bar{u}|^{p-2} \nabla \bar{u}\right)=-\frac{C_{0}}{C} \triangle_{p} \bar{u} \geq \frac{C_{0}}{C} C d^{-\beta}=C_{0} d^{-\beta}
\end{aligned}
$$

Pelo fato de $\phi^{\alpha} \geq\left(\tilde{C}_{1} d\right)^{\alpha}>0$ em $\partial \Omega_{k}$, pois em $\partial \Omega_{k}$ a função $d>0$, temos que $\left(\frac{C_{0}}{C}\right)^{\frac{1}{p-1}} \bar{u}>0=u_{k}$ em $\partial \Omega_{k}$. Pelo princípio da comparação (Teorema 3.2.2), $u_{k} \leq$ $\left(\frac{C_{0}}{C}\right)^{\frac{1}{p-1}} \bar{u}$ em $\Omega_{k}$.

Vamos mostrar que

$$
-\left(\frac{C_{0}}{C}\right)^{\frac{1}{p-1}} \bar{u} \leq u_{k}
$$

De fato

$$
\begin{aligned}
-\triangle_{p}\left(-\left(\frac{C_{0}}{C}\right)^{\frac{1}{p-1}} \bar{u}\right) & =-\operatorname{div}\left(\left|\nabla\left(\frac{C_{0}}{C}\right)^{\frac{1}{p-1}} \bar{u}\right|^{p-2} \nabla\left(-\left(\frac{C_{0}}{C}\right)^{\frac{1}{p-1}} \bar{u}\right)\right) \\
=-\left(-\frac{C_{0}}{C}\right) \operatorname{div}\left(|\nabla \bar{u}|^{p-2} \nabla \bar{u}\right) & =-\frac{C_{0}}{C} \operatorname{div}\left(-|\nabla \bar{u}|^{p-2} \nabla \bar{u}\right)=-\frac{C_{0}}{C}\left(-\triangle_{p} \bar{u}\right) .
\end{aligned}
$$

Como $-\triangle_{p} \bar{u} \geq C d^{-\beta}$ temos

$$
-\frac{C_{0}}{C}\left(-\triangle_{p} \bar{u}\right) \leq-\frac{C_{0}}{C} C d^{-\beta}=-C_{0} d^{-\beta} \leq f=-\triangle_{p} u_{k} .
$$


Assim

$$
\begin{cases}-\triangle_{p}\left(-\left(\frac{C_{0}}{C}\right)^{\frac{1}{p-1}} \bar{u}\right) \leq-\triangle_{p} u_{k}, & x \in \Omega_{k}, \\ -\left(\frac{C_{0}}{C}\right)^{\frac{1}{p-1}} \bar{u} \leq 0=u_{k} & x \in \partial \Omega_{k} .\end{cases}
$$

Pelo princípio da comparação

$$
-\left(\frac{C_{0}}{C}\right)^{\frac{1}{p-1}} \bar{u} \leq u_{k}, \quad x \in \Omega_{k}
$$

Usando (2.8)

$$
\left|u_{k}\right| \leq\left(\frac{C_{0}}{C}\right)^{\frac{1}{p-1}} \bar{u}=\left(\frac{C_{0}}{C}\right)^{\frac{1}{p-1}} \phi^{\frac{p-\beta}{p-1}} \leq \frac{C_{0}^{\frac{1}{p-1}}}{C^{\frac{1}{p-1}}} \tilde{C}_{2}^{\frac{p-\beta}{p-1}} d^{\frac{p-\beta}{p-1}}=\tilde{C} C_{0}^{\frac{1}{p-1}} d^{\frac{p-\beta}{p-1}}
$$

onde $\tilde{C}$ não depende de $k$ ou $f$. Note que $\Omega_{k} \subsetneq \Omega_{k+1} \subsetneq \Omega$ e $\cup_{k=1}^{\infty} \Omega_{k}=\Omega$ e $\left|u_{k}\right| \leq$ $\tilde{C} C_{0}^{\frac{1}{p-1}} d^{\frac{p-\beta}{p-1}} \forall k \in \mathbb{N}$. Neste caso, considere $u_{n k}=\left.u_{n}\right|_{\Omega_{k}}, n \geq k$, onde $u_{n}$ é solução do problema



Portanto em $\Omega_{k}$

$$
\left|u_{n k}\right| \leq C C_{0}^{\frac{1}{p-1}} d^{\frac{p-\beta}{p-1}} \leq \tilde{C} \forall n \geq k
$$

Como $C^{1, \eta}\left(\bar{\Omega}_{k}\right)$ é reflexivo existe uma subsequência $\left\{u_{n_{j}, k}\right\} \subset\left\{u_{n k}\right\}$ que converve fracamente para $u^{k}$ quando $n_{j} \rightarrow \infty$, como $C^{1, \eta}\left(\bar{\Omega}_{k}\right) \stackrel{c p t}{\hookrightarrow} C^{1}\left(\bar{\Omega}_{k}\right)$ por [1], temos $u_{n_{j}, k} \rightarrow u^{k}$ forte em $\Omega_{k}$. Assim

$$
u_{n_{1}, 1} \quad u_{n_{2}, 1} \quad u_{n_{3}, 1} \quad \ldots \rightarrow u^{1} \text { em } C^{1}\left(\bar{\Omega}_{1}\right)
$$

a partir de $u_{n_{1}, 1}$ todas as funções acima estão definida em $\bar{\Omega}_{2}$ então fazendo o mesmo processo acima para a subsequência de (2.25), obtemos uma subsequência de (2.25)

$$
u_{n_{1}, 2} \quad u_{n_{2}, 2} \quad u_{n_{3}, 2} \quad \ldots \quad \rightarrow \quad u^{2} \quad \text { em } \quad C^{1}\left(\bar{\Omega}_{2}\right)
$$

Tomando uma subsequência de (2.26) obtemos

$$
u_{n_{1}, 3} \quad u_{n_{2}, 3} \quad u_{n_{3}, 3} \quad \ldots \quad \rightarrow \quad u^{3} \text { em } C^{1}\left(\bar{\Omega}_{3}\right)
$$

Esse processo é conhecido como processo diagonal. 
Definimos $u(x)=u^{k}(x) \operatorname{com} x \in \Omega_{k}, u$ está bem definida pois $u^{k}(x)=u^{k+1}(x) \operatorname{com} x \in$ $\Omega_{k}$ então $u(x)=\lim _{k \rightarrow \infty} u_{n_{k}, k}(x)$ em $C^{1}(\Omega)$. Agora dado $\phi \in W_{0}^{1, p}(\Omega)$, supp $\phi \subset \Omega$ e existe $k \in \mathbb{N}$ tal que $\Omega_{k} \supset \operatorname{supp} \phi$, assim

$$
\int_{\Omega}\left|\nabla u_{n_{k}, k}\right|^{p-2} \nabla u_{n_{k}, k} \nabla \phi=\int_{\Omega_{k}}\left|\nabla u_{n_{k}, k}\right|^{p-2} \nabla u_{n_{k}, k} \nabla \phi=\int_{\Omega_{k}} f \phi d x=\int_{\Omega} f \phi d x
$$

passando ao limite de $k \rightarrow \infty$, obtemos:

$$
\int_{\Omega}|\nabla u|^{p-2} \nabla u \nabla \phi=\int_{\Omega} f \phi d x
$$

Concluindo, assim a Afirmação 3 .

AfirmaÇ̃̃o 4: $u(x)=0$ em $\partial \Omega$

Tome $x_{0} \in \partial \Omega$, existe uma sequência $\left(x_{n}\right) \subset \Omega$ tal que $x_{n} \rightarrow x_{0}$. Tomando $\Omega_{k} \subset \Omega$ tal que $x_{l} \in \partial \Omega_{k}$ sabemos que $u_{k}=0$ em $\partial \Omega_{k}$ e existe uma subsequência $u_{n_{k}, k} \rightarrow u$

$$
0=\lim _{k, l \rightarrow \infty} u_{n_{k}, k}\left(x_{l}\right)=\lim _{l \rightarrow \infty} u\left(x_{l}\right)=u\left(x_{0}\right)
$$

então $u(x)=0$ em $\partial \Omega$.

Para provar a unicidade seja $u$ e $v$ satisfazem (2.4), usando o princípio da comparação duas vezes

$$
\begin{aligned}
& \left\{\begin{array}{l}
-\triangle_{p} u=f, \quad x \in \Omega, \\
u=0, \quad x \in \partial \Omega,
\end{array}\right. \\
& \left\{\begin{array}{l}
-\triangle_{p} v=f, \quad x \in \Omega, \\
v=0, \quad x \in \partial \Omega,
\end{array}\right.
\end{aligned}
$$

temos $u=v$ em $\Omega$. Garantindo, assim, a unicidade.

b) Para provar o caso $0<\beta \leq 1$ observe se $|f| \leq C_{0} d^{-\beta}$ então

$$
|f| \leq C_{0} d^{-\beta}=C_{0} d^{-\theta} d^{\theta-\beta} \leq C d^{-\theta}
$$

para $\theta>\beta$ então $|f| \leq C d^{-\theta}$ para $\theta \in(1, p)$ usando o caso a), garantimos a existência da solução $u$ para o problema (2.4).

Os próximos resultados serão importantes para provar a estimativa (2.3).

Lema 2.1.1 Sejam $q>p-1, Q_{0}>0$ e $\gamma<p$ e $u \in W_{l o c}^{1, p}(0, \infty)$ uma solução fraca 
positiva do problema

$$
\left\{\begin{array}{r}
\left(\left|u^{\prime}\right|^{p-2} u^{\prime}\right)^{\prime}=Q_{0} x^{-\gamma} u^{q}, \quad \text { com } x>0, \\
u(0)=+\infty
\end{array}\right.
$$

então

$$
u(x)=\left(\frac{(p-1) \alpha^{p-1}(\alpha+1)}{Q_{0}}\right)^{\frac{1}{q-p+1}} x^{-\alpha}
$$

onde $\alpha=\frac{p-\gamma}{q-p+1}$.

Prova: Vamos verificar, primeiramente, que toda solução de (2.31) satisfaz

$$
C_{1} x^{-\alpha} \leq u \leq C_{2} x^{-\alpha} \text { com } x>0
$$

para constantes positivas $C_{1}, C_{2}$. Para isso, fixe $x>0$ e considere

$$
v(y)=x^{\alpha} u(x+x y) \quad \text { com }|y|<\frac{1}{2} .
$$

Observe que

$$
v^{\prime}(y)=\frac{d}{d y}\left(x^{\alpha} u(x+x y)\right)=x^{\alpha+1} \frac{d u}{d z}(z)=x^{\alpha+1} u^{\prime}(z)
$$

onde $z=x+x y$,

$$
\begin{aligned}
\left|v^{\prime}(y)\right|^{p-2} & =x^{(\alpha+1)(p-2)}\left|u^{\prime}(z)\right|^{p-2} \\
\left|v^{\prime}(y)\right|^{p-2} v^{\prime}(y) & =x^{(\alpha+1)(p-1)}\left|u^{\prime}(z)\right|^{p-2} u^{\prime}(z)
\end{aligned}
$$

$\log 0$ 


$$
\begin{aligned}
\left(\left|v^{\prime}(y)\right|^{p-2} v^{\prime}(y)\right)^{\prime} & =\left[x^{(\alpha+1)(p-1)}\left|u^{\prime}(z)\right|^{p-2} u^{\prime}(z)\right]^{\prime} \\
& =x^{(\alpha+1)(p-1)} \frac{d}{d y}\left[\left|u^{\prime}(z)\right|^{p-2} u^{\prime}(z)\right] \\
& =x^{(\alpha+1)(p-1)} \frac{d}{d(z)}\left[\left|u^{\prime}(z)\right|^{p-2} u^{\prime}(z)\right] \frac{d(z)}{d(y)} \\
& =x^{(\alpha+1)(p-1)} Q_{0}(x+x y)^{-\gamma} u^{q}(x+x y) x \\
& =x^{[(\alpha+1)(p-1)-\gamma+1]} Q_{0}(1+y)^{-\gamma} u^{q}(x+x y) \\
& =x^{([\alpha+1)(p-1)-\gamma+1]} Q_{0}(1+y)^{-\gamma} v^{q}(y) x^{-\alpha q} \\
& =x^{[(\alpha+1)(p-1)-\gamma+1-\alpha q]} Q_{0}(1+y)^{-\gamma} v^{q}(y) .
\end{aligned}
$$

Por outro lado,

$$
\begin{aligned}
(\alpha+1)(p-1)-\gamma+1-\alpha q & =\alpha p-\alpha+p-1-\gamma+1-\alpha q=-\alpha(q-p+1)+p-\gamma \\
& =-p+\gamma+p-\gamma=0
\end{aligned}
$$

Sendo assim

$$
\left(\left|v^{\prime}(y)\right|^{p-2} v^{\prime}(y)\right)^{\prime}=Q_{0}(1+y)^{-\gamma} v^{q}(y) \quad \text { com }|y|<\frac{1}{2} .
$$

Seja $U$ a solução de

$$
\left\{\begin{array}{l}
\left(\left|U^{\prime}\right|^{p-2} U^{\prime}\right)^{\prime}=Q_{0}(1+y)^{-\gamma} U^{q}, \quad|y|<\frac{1}{2} \\
U\left( \pm \frac{1}{2}\right)=+\infty
\end{array}\right.
$$

O problema (2.35) possui solução positiva, uma vez que $Q_{0}(1+y)^{-\gamma}>0$ com $|y|<\frac{1}{2} \mathrm{e}$ $f(U)=U^{q}$ satisfaz a condição de Keller-Osserman (ver Apêndice A), então pelo Teorema (F.1) do Anexo 1 o problema (2.35) tem solução. Seja $U$ essa solução. Como

$$
\left\{\begin{aligned}
\left(\left|v^{\prime}\right|^{p-2} v^{\prime}\right)^{\prime} & =Q_{0}(1+y)^{-\gamma} v^{q}(y), \\
\left(\left|U^{\prime}\right|^{p-2} U^{\prime}\right)^{\prime} & =Q_{0}(1+y)^{-\gamma} U^{q}(y), \\
v\left(\frac{1}{2}\right) & =x^{\alpha} u\left(\frac{3 x}{2}\right) \leq U\left(\frac{1}{2}\right)=+\infty \\
v\left(-\frac{1}{2}\right) & =x^{\alpha} u\left(\frac{x}{2}\right) \leq U\left(\frac{-1}{2}\right)=+\infty
\end{aligned}\right.
$$

pelo princípio da comparação $v \leq U$ em $\left\{y \in \mathbb{R} /|y| \leq \frac{1}{2}\right\}$. Tomando $y=0$ obtemos $x^{\alpha} u(x) \leq U(0) \Rightarrow u(x) \leq x^{-\alpha} U(0)$. A constante $U(0)$ é positiva pois do Teorema (1.6.1), temos $U>0$ para $y \in\left[-\frac{1}{2}, \frac{1}{2}\right]$ então $U(0)>0$. 
Agora iremos provar o lado esquerdo da desigualdade (2.33). Para isso, seja $V$ a solução para o problema

$$
\left\{\begin{array}{l}
\left(\left|V^{\prime}\right|^{p-2} V^{\prime}\right)^{\prime}=Q_{0}(1+y)^{-\gamma} V^{q}, \quad|y|<1 \\
V(-1)=1 \quad V(1)=0
\end{array}\right.
$$

O problema (2.37) tem solução, pois $\underline{v}=0$ é subsolução e $\bar{v}=1$ é supersolução. Então como

$$
\left\{\begin{aligned}
\left(\left|V^{\prime}\right|^{p-2} V^{\prime}\right)^{\prime} & =Q_{0}(1+y)^{-\gamma} V^{q} \\
\left(\left|v^{\prime}\right|^{p-2} v^{\prime}\right)^{\prime} & =Q_{0}(1+y)^{-\gamma} v^{q}(y), \\
v(1) & =x^{\alpha} u(2 x) \geq 0=V(1), \\
v(-1) & =x^{\alpha} u(0)=+\infty \geq 1=V(-1),
\end{aligned}\right.
$$

pelo princípio da comparação $v \geq V \quad \Rightarrow \quad V(0) \leq v(0)=x^{\alpha} u(x) \quad \Rightarrow \quad x^{-\alpha} V(0) \leq u(x)$. A constante $V(0)>0$, pois do Teorema (1.6.1), temos $V(y)>0$ para $y \in[-1,1]$, então $V(0)>0$.

Então conseguimos mostrar que

$$
V(0) x^{-\alpha} \leq u(x) \leq U(0) x^{-\alpha}
$$

Pelo Lema (F.2)

$$
\left(\left|u^{\prime}(x)\right|^{p-2} u^{\prime}(x)-\left|u^{\prime}(y)\right|^{p-2} u^{\prime}(y)\right) \cdot\left(u^{\prime}(x)-u^{\prime}(y)\right)>0
$$

se $x<y$, por hipótese $\left|u^{\prime}(x)\right|^{p-2} u^{\prime}(x)$ é não decrescente, segue que $u^{\prime}(x)-u^{\prime}(y)<0 \Rightarrow$ $u^{\prime}(x)<u^{\prime}(y)$ então $u^{\prime}$ é crescente. De (2.33) temos que $\lim _{x \rightarrow \infty} u(x)=0$, e do teorema do valor médio seja $\delta \in(x, 2 x)$ temos

$$
u^{\prime}(\delta)=\frac{u(2 x)-u(x)}{x} \Rightarrow \lim _{\delta \rightarrow \infty} u^{\prime}(\delta)=\lim _{x \rightarrow \infty}[u(2 x)-u(x)] \frac{1}{x}=0 .
$$

já que $u^{\prime}(x)$ é crescente, concluimos $u^{\prime}<0$. Temos de $(2.33)$

$$
\left(\left|u^{\prime}\right|^{p-2} u^{\prime}\right)^{\prime}=Q_{0} x^{-\gamma} u^{q} \leq Q_{0} x^{-\gamma} C_{2}^{q} x^{-\alpha q}=Q_{0} C_{2}^{q} x^{-(\gamma+\alpha q)}=Q_{0} C_{2}^{q} x^{-(\alpha+1)(p-1)-1}
$$

pois 


$$
\begin{aligned}
(\alpha+1)(p-1)-\gamma-\alpha q+1 & =\alpha p-\alpha+p-1-\gamma-\alpha q+1 \\
& =\alpha p-\alpha+p-\gamma-\alpha q \\
& =-\alpha(q-p+1)-\gamma+p \\
& =-p+\gamma-\gamma+p=0 \Rightarrow \\
-(\gamma+\alpha q) & =-(\alpha+1)(p-1)-1
\end{aligned}
$$

Integrando (2.39)

$$
\begin{aligned}
\int_{x}^{y}\left(\left|u^{\prime}(t)\right|^{p-2} u^{\prime}(t)\right)^{\prime} d t & \leq Q_{0} C_{2}^{q} \int_{x}^{y} t^{-(\alpha+1)(p-1)-1} d t \Rightarrow \\
\left|u^{\prime}(y)\right|^{p-2} u^{\prime}(y)-\left|u^{\prime}(x)\right|^{p-2} u^{\prime}(x) & \leq \frac{Q_{0} C_{2}^{q}}{-(\alpha+1)(p-1)}\left[y^{-(\alpha+1)(p-1)}-x^{-(\alpha+1)(p-1)}\right]
\end{aligned}
$$

fazendo $y \rightarrow+\infty$ temos

$$
-\left|u^{\prime}(x)\right|^{p-2} u^{\prime}(x) \leq \frac{Q_{0} C_{2}^{q}}{(\alpha+1)(p-1)} x^{-(\alpha+1)(p-1)}=C x^{-(\alpha+1)(p-1)}
$$

$\forall x>0$. Pelo fato de $u^{\prime}(x)<0$ em $(0,+\infty)$ temos $\left|u^{\prime}(x)\right|=-u(x)$, assim

$$
\begin{aligned}
-\left|u^{\prime}(x)\right|^{p-2} u^{\prime}(x) & =\left|u^{\prime}(x)\right|^{p-2}\left|u^{\prime}(x)\right| \leq C x^{-(\alpha+1)(p-1)} \Rightarrow\left|u^{\prime}(x)\right|^{p-1} \leq C x^{-(\alpha+1)(p-1)} \\
\left|u^{\prime}(x)\right| & \leq C x^{-(\alpha+1)} \Rightarrow-u^{\prime}(x) \leq C x^{-(\alpha+1)} \quad \forall x>0 .
\end{aligned}
$$

Por outro lado,

$$
\begin{aligned}
\left(\left|u^{\prime}(x)\right|^{p-2} u^{\prime}(x)\right)^{\prime} & =Q_{0} x^{-\gamma} u^{q} \geq Q_{0} x^{-\gamma} C_{1}^{q} x^{-\alpha q}=Q_{0} x^{-(\gamma+\alpha q)} C_{1}^{q} \\
& =Q_{0} x^{-(\alpha+1)(p-1)-1} C_{1}^{q}
\end{aligned}
$$

Integrando (2.42) para $x<y$, temos

$$
\int_{x}^{y}\left(\left|u^{\prime}(t)\right|^{p-2} u^{\prime}(t)\right)^{\prime} d t \geq \int_{x}^{y} Q_{0} C_{1}^{q} t^{-(\alpha+1)(p-1)-1} d t
$$

donde

$$
\begin{aligned}
\left|u^{\prime}(y)\right|^{p-2} u^{\prime}(y)-\left|u^{\prime}(x)\right|^{p-2} u^{\prime}(x) & \geq \frac{Q_{0} C_{1}^{q}}{(\alpha+1)(p-1)}\left[x^{-(\alpha+1)(p-1)}-y^{-(\alpha+1)(p-1)}\right] \Rightarrow \\
\left|u^{\prime}(y)\right|^{p-2} u^{\prime}(y)-\left|u^{\prime}(x)\right|^{p-2} u^{\prime}(x) & \geq \hat{C}\left[x^{-(\alpha+1)(p-1)}-y^{-(\alpha+1)(p-1)}\right]
\end{aligned}
$$


tomando $y \rightarrow+\infty$

$$
-\left|u^{\prime}(x)\right|^{p-2} u^{\prime}(x) \geq \hat{C} x^{-(\alpha+1)(p-1)}, \quad \forall x>0 .
$$

Desde que $u^{\prime}(x)<0$ em $(0, \infty)$, tem-se

$$
\begin{aligned}
-\left|u^{\prime}(x)\right|^{p-2} u^{\prime}(x) & =\left|u^{\prime}(x)\right|^{p-1} \geq \hat{C} x^{-(\alpha+1)(p-1)} \Rightarrow\left|u^{\prime}(x)\right| \geq \hat{C} x^{-(\alpha+1)} \Rightarrow \\
-u^{\prime}(x) & \geq \hat{C} x^{-(\alpha+1)} .
\end{aligned}
$$

De (2.41) e (2.43) temos

$$
\hat{C} x^{-(\alpha+1)} \leq-u^{\prime}(x) \leq C x^{-(\alpha+1)} \quad \forall x>0 .
$$

Uma vez que $u^{\prime}<0$ e $u \in C^{2}(0,+\infty)$ temos

$$
\begin{aligned}
& Q_{0} x^{-\gamma} u^{q}=\left(\left|u^{\prime}\right|^{p-2} u^{\prime}\right)^{\prime}=\left(\left(-u^{\prime}\right)^{p-2} u^{\prime}\right)^{\prime}=(p-2)\left(-u^{\prime}\right)^{p-3}\left(-u^{\prime \prime}\right) u^{\prime}+\left(\left(-u^{\prime}\right)^{p-2} u^{\prime \prime}\right) \\
&=(p-2)\left(-u^{\prime}\right)^{p-3}\left(u^{\prime \prime}\right)\left(-u^{\prime}\right)+\left(-u^{\prime}\right)^{p-2} u^{\prime \prime}=(p-2)\left(-u^{\prime}\right)^{p-2} u^{\prime \prime}+\left(-u^{\prime}\right)^{p-2} u^{\prime \prime} \\
&=(p-1)\left(-u^{\prime}\right)^{p-2} u^{\prime \prime}=(p-1)\left|u^{\prime}\right|^{p-2} u^{\prime \prime} \Rightarrow \\
& Q_{0} x^{-\gamma} u^{q}=(p-1)\left|u^{\prime}\right|^{p-2} u^{\prime \prime}
\end{aligned}
$$

Considerando $u=x^{-\alpha} v$,

$$
\begin{aligned}
u^{\prime}(x) & =-\alpha x^{-\alpha-1} v+x^{-\alpha} v^{\prime} \\
u^{\prime \prime}(x) & =\alpha(\alpha+1) x^{-\alpha-2} v-\alpha x^{-\alpha-1} v^{\prime}-\alpha x^{-\alpha-1} v^{\prime}+x^{-\alpha} v^{\prime \prime}
\end{aligned}
$$

Assim substituindo em (2.45) temos

$$
\begin{gathered}
Q_{0} x^{-\gamma} x^{-\alpha q} v^{q}=(p-1)\left|-\alpha x^{-(\alpha+1)} v+x^{-\alpha} v^{\prime}\right|^{p-2}\left[\alpha(\alpha+1) x^{-(\alpha+2)} v-2 \alpha x^{-(\alpha+1)} v^{\prime}+x^{-\alpha} v^{\prime \prime}\right] \\
Q_{0} x^{-(\gamma+\alpha q)} v^{q}=(p-1) x^{-(\alpha+1)(p-2)}\left|-\alpha v+x v^{\prime}\right|^{p-2} x^{-(\alpha+2)}\left[\alpha(\alpha+1) v-2 \alpha x v^{\prime}+x^{2} v^{\prime \prime}\right] .
\end{gathered}
$$

Além disso,

$$
\begin{aligned}
-(\alpha+1)(p-2)-(\alpha+2) & =-\alpha p+2 \alpha-p+2-\alpha-2=-\alpha p+\alpha-p \\
& =-\alpha p+\alpha+\alpha q-\alpha q-p=\alpha(q-p+1)-p-\alpha q \\
& =p-\gamma-p-\alpha q=-(\gamma+\alpha q) .
\end{aligned}
$$


Logo,

$$
\begin{aligned}
Q_{0} x^{-(\gamma+\alpha q)} v^{q} & =(p-1) x^{-(\gamma+\alpha q)}\left|-\alpha v+x v^{\prime}\right|^{p-2}\left[\alpha(\alpha+1) v-2 \alpha x v^{\prime}+x^{2} v^{\prime \prime}\right] \\
Q_{0} v^{q} & =(p-1)\left|-\alpha v+x v^{\prime}\right|^{p-2}\left[\alpha(\alpha+1) v-2 \alpha x v^{\prime}+x^{2} v^{\prime \prime}\right] .
\end{aligned}
$$

A função $v$ é limitada, pois de (2.33) temos

$$
C_{1} x^{-\alpha} \leq u(x) \leq C_{2} x^{-\alpha} \Rightarrow C_{1} \leq u(x) x^{\alpha} \leq C_{2} \Rightarrow C_{1} \leq v(x) \leq C_{2}
$$

Fazendo a mudança de variável $t=-\alpha \log x$ e $w(t)=v(x)$ a equação (2.46) com a nova mudança fica

$$
\begin{aligned}
v^{\prime}(x) & =\frac{d w(t)}{d x}=\frac{d w(t)}{d t} \frac{d t}{d x}=w^{\prime}(t)\left(\frac{-\alpha}{x}\right)=-\alpha x^{-1} w^{\prime}(t) \\
v^{\prime \prime}(x) & =\frac{d v^{\prime}(x)}{d x}=\frac{d\left(-\alpha x^{-1} w^{\prime}(t)\right)}{d x}=\alpha x^{-2} w^{\prime}(t)-\alpha x^{-1} \frac{d w^{\prime}(t)}{d x} \\
& =\alpha x^{-2} w^{\prime}(t)-\alpha x^{-1} w^{\prime \prime}(t)\left(\frac{-\alpha}{x}\right)=\alpha x^{-2} w^{\prime}(t)+\alpha^{2} x^{-2} w^{\prime \prime}(t) .
\end{aligned}
$$

E substituindo em (2.46)

$$
\begin{aligned}
& Q_{0} w^{q}(t)=(p-1)\left[\alpha w(t)+x \alpha x^{-1} w^{\prime}(t)\right]^{p-2}\left[\alpha(\alpha+1) w(t)+2 \alpha^{2} w^{\prime}(t)+\alpha w^{\prime}(t)+\alpha^{2} w^{\prime \prime}(t)\right] \\
& =(p-1)\left[\alpha w(t)+\alpha w^{\prime}(t)\right]^{p-2} \alpha\left[(\alpha+1) w(t)+2 \alpha w^{\prime}(t)+w^{\prime}(t)+\alpha w^{\prime \prime}(t)\right] \\
& =(p-1) \alpha^{p-1}\left(w+w^{\prime}\right)^{p-2}\left[\alpha w^{\prime \prime}+(2 \alpha+1) w^{\prime}+(\alpha+1) w\right] \quad \text { em } \quad \mathbb{R} .
\end{aligned}
$$

Tendo em vista que

$$
\begin{aligned}
u(x) & =x^{-\alpha} v(x) \\
u^{\prime}(x) & =-\alpha x^{-\alpha-1} v(x)+x^{-\alpha} v^{\prime}(x)=-\alpha x^{-(\alpha+1)} w(t)+x^{-\alpha}\left(-\alpha x^{-1} w^{\prime}(t)\right) \\
& =-\alpha x^{-(\alpha+1)} w(t)-\alpha x^{-(\alpha+1)} w^{\prime}(t)=-\alpha x^{-(\alpha+1)}\left[w(t)+w^{\prime}(t)\right]
\end{aligned}
$$

segue de (2.44) que

$$
\begin{aligned}
C_{1} x^{-(\alpha+1)} & \leq-(-\alpha) x^{-(\alpha+1)}\left[w(t)+w^{\prime}(t)\right] \leq C_{2} x^{-(\alpha+1)} \Rightarrow \\
\hat{C}_{1} & \leq w(t)+w^{\prime}(t) \leq \hat{C}_{2} .
\end{aligned}
$$

Já que $v$ é uma função limitada temos $w$ é limitada e de (2.48) obtemos que $w^{\prime}$ é limitada. 
Nosso objetivo é mostrar que $w=A$, onde

$$
A=\left[\frac{(p-1) \alpha^{p-1}(\alpha+1)}{Q_{0}}\right]^{\frac{1}{q-p+1}} .
$$

Para isso vamos provar primeiramente que

$$
\lim _{t \rightarrow \pm \infty} w(t)=A
$$

Observe que (2.47) não tem solução periódica, limitada em $\mathbb{R}$ exceto a constante $A$. De fato, se $w$ é periódica, seja $I$ um intervalo compacto que contém o período de $w$, então $w$ possui valores máximo e mínimo em $I$. Que são os valores máximo e mínimo global. Seja $t_{1}$ ponto de máximo e $t_{2}$ o ponto de mínimo. Então $w^{\prime}\left(t_{1}\right)=w^{\prime}\left(t_{2}\right)=0 \mathrm{e}$ $w^{\prime \prime}\left(t_{1}\right) \leq 0$ e $w^{\prime \prime}\left(t_{2}\right) \geq 0$. De (2.47) segue que, para $t=t_{1}$

$$
\begin{aligned}
Q_{0} w^{q}\left(t_{1}\right) & =(p-1) \alpha^{p-1}\left[w\left(t_{1}\right)\right]^{p-2}\left[\alpha w^{\prime \prime}\left(t_{1}\right)+(\alpha+1) w\left(t_{1}\right)\right] \leq(p-1) \alpha^{p-1}(\alpha+1) w\left(t_{1}\right)^{p-1}, \\
w\left(t_{1}\right)^{q-p+1} & \leq \frac{(p-1) \alpha^{p-1}(\alpha+1)}{Q_{0}} \Rightarrow w\left(t_{1}\right) \leq\left(\frac{(p-1) \alpha^{p-1}(\alpha+1)}{Q_{0}}\right)^{\frac{1}{q-p+1}} .
\end{aligned}
$$

Para $t=t_{2}$

$$
\begin{aligned}
Q_{0} w^{q}\left(t_{2}\right) & =(p-1) \alpha^{p-1}\left[w\left(t_{2}\right)\right]^{p-2}\left[\alpha w^{\prime \prime}\left(t_{2}\right)+(\alpha+1) w\left(t_{2}\right)\right] \geq(p-1) \alpha^{p-1}(\alpha+1) w\left(t_{2}\right)^{p-1}, \\
w\left(t_{2}\right)^{q-p+1} & \geq \frac{(p-1) \alpha^{p-1}(\alpha+1)}{Q_{0}} \Rightarrow w\left(t_{2}\right) \geq\left[\frac{(p-1) \alpha^{p-1}(\alpha+1)}{Q_{0}}\right]^{\frac{1}{q-p+1}} .
\end{aligned}
$$

De $(2.51)$ e (2.52) temos

$$
w\left(t_{1}\right) \leq A \leq w\left(t_{2}\right)
$$

e isso só ocorre se $w(t)=A$.

Podemos reescrever (2.47) como o sistema autônomo de equações diferenciais

$$
\left\{\begin{array}{l}
w^{\prime}(t)=z(t) \\
z^{\prime}(t)=\frac{Q_{0} w^{q}(t)}{\alpha^{p}(p-1)(z+w)^{p-2}}-\frac{(2 \alpha+1) z}{\alpha}-\frac{(\alpha+1) w}{\alpha},
\end{array}\right.
$$

e observe que toda solução $w$ de (2.47) verifica (2.48) nos dando então uma solução limitada do sistema (2.54). 
Como (2.47) não tem solução periódica então pelo teorema de Poincaré-Bendixon $\omega(p)$ contém pontos singulares, mas como o único ponto singular de $(2.54)$ é $(A, 0)$ pois

$$
\begin{aligned}
w^{\prime}(t) & =0 \Rightarrow z(t)=0, \\
z^{\prime}(t) & =0 \Rightarrow \frac{Q_{0} w^{q}}{\alpha^{p}(p-1) w^{p-2}}-\frac{(\alpha+1) w}{\alpha}=0 \Rightarrow Q_{0} w^{q}=(\alpha+1) \alpha^{p-1}(p-1) w^{p-1} \Rightarrow \\
w^{q-p+1} & =\frac{(\alpha+1) \alpha^{p-1}(p-1)}{Q_{0}} \Rightarrow w=\left(\frac{(\alpha+1) \alpha^{p-1}(p-1)}{Q_{0}}\right)^{\frac{1}{q-p+1}}=A
\end{aligned}
$$

então $(A, 0) \in \omega(p)$. Pela definição de $\omega(p)$ temos se $t_{n} \rightarrow \infty$ quando $n \rightarrow \infty$ temos $\left(w\left(t_{n}\right), z\left(t_{n}\right)\right) \rightarrow(A, 0)$, ou seja, quando $t \rightarrow \infty$ temos $(w(t), z(t)) \rightarrow(A, 0)$. Segue de (2.50) que

$$
\lim _{t \rightarrow \pm \infty} w(t)=A
$$

Assuma que $\sup w>A$. De (2.50), existe $t_{0} \in \mathbb{R}$ tal que $w$ atinge o máximo global em $t_{0}$. E análogo a prova de (2.53) obtemos $w\left(t_{0}\right) \leq A<\sup w$ absurdo. Então $\sup w \leq A$.

Suponha inf $w<A$. De (2.50) temos que existe $t_{1} \in \mathbb{R}$ tal que $w$ atinge o mínimo global em $t_{1}$. E análogo a prova de (2.53) obtemos $w\left(t_{1}\right) \geq A>\inf w$ absurdo. Então inf $w \geq A$. Logo

$$
\begin{array}{r}
\sup w \leq A \leq \inf w \Rightarrow w=A \Rightarrow w(t)=v(x)=A \Rightarrow \\
u(x)=x^{-\alpha} A=\left[\frac{(p-1) \alpha^{p-1}(\alpha+1)}{Q_{0}}\right]^{\frac{1}{q-p+1}} x^{-\alpha}
\end{array}
$$

O próximo teorema será importante na prova da estimativa do Teorema 2.1. Usaremos, para provar o teorema a seguir, o Lema 2.1.1.

O próximo problema está definido em um espaço $D=\left\{x \in \mathbb{R}^{n} ; x_{1}>0\right\}$, onde $x$ pode ser escrito como $x=\left(x_{1}, x^{\prime}\right)$.

Teorema 2.1.2 Seja $Q_{0}>0, \gamma<p, q>p-1$ e $u \in W_{l o c}^{1, p}(D)$ uma solução positiva do problema

$$
\left\{\begin{array}{l}
\triangle_{p} u=Q_{0} x_{1}^{-\gamma} u^{q}, \quad x \in D \\
u=+\infty, \quad x \in \partial D
\end{array}\right.
$$

Então

$$
u(x)=\left(\frac{(p-1) \alpha^{p-1}(\alpha+1)}{Q_{0}}\right)^{\frac{1}{q-p+1}} x_{1}^{-\alpha}
$$

onde $\alpha=\frac{p-\gamma}{q-p+1}$ e $D=\left\{x \in \mathbb{R}^{n} ; x_{1}>0\right\}$. 
Prova: Denote por $u_{0}$ a solução unidimensional do problema (2.56) dado por

$$
u_{0}(x)=\left(\frac{(p-1) \alpha^{p-1}(\alpha+1)}{Q_{0}}\right)^{\frac{1}{q-p+1}} x_{1}^{-\alpha}
$$

$u_{0}$ é solução de (2.56) pois

$$
\begin{aligned}
\nabla u_{0}(x) & =\left(\left(\frac{(p-1) \alpha^{p-1}(\alpha+1)}{Q_{0}}\right)^{\frac{1}{q-p+1}}(-\alpha) x_{1}^{-(\alpha+1)}, 0, \ldots, 0\right), \\
\left|\nabla u_{0}(x)\right| & =\alpha\left(\frac{(p-1) \alpha^{p-1}(\alpha+1)}{Q_{0}}\right)^{\frac{1}{q-p+1}}\left|x_{1}\right|^{-(\alpha+1)}, \\
\left|\nabla u_{0}(x)\right|^{p-2} \nabla u_{0}(x) & =-\alpha^{p-1}\left(\frac{(p-1) \alpha^{p-1}(\alpha+1)}{Q_{0}}\right)^{\frac{p-1}{q-p+1}} x_{1}^{-(\alpha+1)(p-1)}(1,0,0, \ldots, 0), \\
\operatorname{div}\left(\left|\nabla u_{0}(x)\right|^{p-2} \nabla u_{0}(x)\right) & =(\alpha+1)(p-1) \alpha^{p-1}\left(\frac{(p-1) \alpha^{p-1}(\alpha+1)}{Q_{0}}\right)^{\frac{p-1}{q-p+1}} x_{1}^{-(\alpha+1)(p-1)-1} \\
& =\frac{\left((\alpha+1)(p-1) \alpha^{p-1}\right)^{\frac{q}{q-p+1}} x_{1}^{-\gamma-\alpha q}}{Q_{0}^{\frac{p-1}{q-p+1}}} \\
& =\frac{Q_{0} x_{1}^{-\gamma}\left((\alpha+1)(p-1) \alpha^{p-1}\right)^{\frac{q}{q-p+1}} x^{-\alpha q}}{Q^{\frac{p-1}{q-p+1}+1}} \\
& =Q_{0} x_{1}^{-\gamma} u_{0}^{q}(x) .
\end{aligned}
$$

Usando essa solução vamos mostrar que o problema (2.56) tem uma solução maximal e minimal as duas dependendo somente da variável $x_{1}$.

Mostraremos, primeiramente, que (2.56) admite solução maximal $u_{\max }$. Seja $\left\{D_{k}\right\}$ uma sequência de domínios limitados suaves tal que $D_{k} \subset \subset D_{k+1}$ e $D=\bigcup_{k=1}^{\infty} D_{k}$. Como $D_{k}$ é um conjunto limitado para todo $k$, temos que $x_{1}^{-\gamma}$ é limitado e $x_{1}^{-\gamma}$ é positiva, o problema

$$
\left\{\begin{array}{l}
\triangle_{p} u=Q_{0} x_{1}^{-\gamma} u^{q}, \quad x \in D_{k} \\
u \quad=+\infty, \quad x \in \partial D_{k}
\end{array}\right.
$$


tem solução fraca positiva $u_{k}$.

A existência de solução em (2.58) é garantida pelo Lema (F.1) do Anexo 1. Onde $g(x)=Q_{0} x_{1}^{-\gamma}$ e $f(u)=u^{q}$ satisfaz a condição de Keller-Osserman. Então o problema (2.58) admite uma solução $u_{k}$ não negativa $u \in W_{l o c}^{1, p}\left(D_{k}\right) \cap C^{1, \beta}\left(D_{k}\right), 0<\beta<1$.

Como $u_{k+1}$ é contínua então $u_{k+1}(x) \leq \infty=u_{k}(x)$ na $\partial D_{k}$ e em $D_{k}$

$$
\begin{array}{r}
\triangle_{p} u_{k+1} \leq Q_{0} x_{1}^{-\gamma} u_{k+1}^{q} \\
\triangle_{p} u_{k} \geq Q_{0} x_{1}^{-\gamma} u_{k}^{q} .
\end{array}
$$

Pelo Princípio da comparação ( ver Teorema (3.2.2)), temos $u_{k+1}(x) \leq u_{k}(x)$ em $D_{k}$. Do mesmo modo $u_{0}(x) \leq \infty=u_{k}(x)$ na $\partial D_{k}$

$$
\begin{aligned}
& \triangle_{p} u_{0} \leq Q_{0} x_{1}^{-\gamma} u_{0}^{q}, \\
& \triangle_{p} u_{k} \geq Q_{0} x_{1}^{-\gamma} u_{k}^{q},
\end{aligned}
$$

$\operatorname{logo} u_{0} \leq u_{k}$ em $D_{k}$. Assim a sequência $\left\{u_{k}\right\}$ é não-crescente e limitada inferiormente, e portanto $u_{k}$ converge, isto é

$$
u_{\max }(x)=\lim _{k \rightarrow \infty} u_{k}(x) \geq u_{0}(x) .
$$

Argumentando como na prova do Teorema (2.1.1) e usando o processo diagonal obtemos que $u_{k}$ converge em $C^{1}\left(D_{k}\right)$ para todo $k$, logo converge em $C^{1}(D)$.

Por isso $u_{\max }$ é solução fraca para (2.56).

AfirmaÇÃo $1 u_{\max }$ é solução maximal

Suponha que $v$ é outra solução de (2.56), segue

$$
\begin{aligned}
v(x) \leq u_{k}(x) & =\infty, \quad \forall x \in \partial D_{k}, \\
\triangle_{p} v & \leq Q_{0} x_{1}^{-\gamma} v^{q}, \\
\triangle_{p} u_{k}(x) & \geq Q_{0} x_{1}^{-\gamma} u_{k}^{q} .
\end{aligned}
$$

Pelo princípio da comparação 
$v \leq u_{k}, \quad$ em $\quad D_{k}, \quad \forall k$

então

$$
\begin{aligned}
v=\lim _{k \rightarrow \infty} v & \leq \lim _{k \rightarrow \infty} u_{k}=u_{\max }, \quad \text { em } \quad D \Rightarrow \\
v & \leq u_{\max }, \quad \text { em } D .
\end{aligned}
$$

Observe que $u_{\max }$ depende apenas de $x_{1}$. De fato, seja $t \in \mathbb{R}^{n-1}$ arbitrário. É fácil ver que a função $w(x)=u_{\max }\left(x_{1}, x^{\prime}+t\right)$ é uma solução de $(2.56)$ e que $w \leq u_{\max }$, pois

$$
\begin{aligned}
\nabla w(x) & =\left(\frac{\partial w(x)}{\partial x_{1}}, \frac{\partial w(x)}{\partial x_{2}}, \ldots, \frac{\partial w(x)}{\partial x_{n}}\right) \\
& =\left(\frac{\partial u_{\max }\left(x_{1}, x^{\prime}+t\right)}{\partial x_{1}}, \frac{\partial u_{\max }\left(x_{1}, x^{\prime}+t\right)}{\partial x_{2}}, \ldots, \frac{\partial u_{\max }\left(x_{1}, x^{\prime}+t\right)}{\partial x_{n}}\right) \\
& =\left(\frac{\partial u_{\max }\left(x_{1}, x^{\prime}+t\right)}{\partial x_{1}}, \frac{\partial u_{\max }\left(x_{1}, x^{\prime}+t\right)}{\partial z_{1}}, \ldots, \frac{\partial u_{\max }\left(x_{1}, x^{\prime}+t\right)}{\partial z_{n-1}}\right) \\
& =\nabla u_{\max }\left(x_{1}, z\right)
\end{aligned}
$$

onde $z=x^{\prime}+t=\left(x_{1}^{\prime}+t_{1}, \ldots, x_{n-1}^{\prime}+t_{n-1}\right) \in \mathbb{R}^{n-1}$.

$$
\begin{aligned}
\operatorname{div}\left(|\nabla w(x)|^{p-2} \nabla w(x)\right) & =\sum_{i=1}^{n} \frac{\partial}{\partial x_{i}}\left(|\nabla w(x)|^{p-2} \frac{\partial w(x)}{\partial x_{i}}\right) \\
& =\frac{\partial}{\partial x_{1}}\left(\left|\nabla u_{\max }\left(x_{1}, z\right)\right|^{p-2} \frac{\partial u_{\max }\left(x_{1}, z\right)}{\partial x_{1}}\right) \\
& +\sum_{i=2}^{n} \frac{\partial}{\partial x_{i}}\left(\left|\nabla u_{\max }\left(x_{1}, z\right)\right|^{p-2} \frac{\partial u_{\max }\left(x_{1}, z\right)}{\partial z_{i-1}}\right) \\
& =\frac{\partial}{\partial x_{1}}\left(\left|\nabla u_{\max }\left(x_{1}, z\right)\right|^{p-2} \frac{\partial u_{\max }\left(x_{1}, z\right)}{\partial x_{1}}\right) \\
& +\sum_{i=2}^{n} \frac{\partial}{\partial z_{i-1}}\left(\left|\nabla u_{\max }\left(x_{1}, z\right)\right|^{p-2} \frac{\partial u_{\max }\left(x_{1}, z\right)}{\partial z_{i-1}}\right) \\
& =\operatorname{div}\left(\left|\nabla u_{\max }\left(x_{1}, z\right)\right|^{p-2} \nabla u_{\max }\left(x_{1}, z\right)\right),
\end{aligned}
$$

ou seja ,

$$
\triangle_{p} w(x)=\triangle_{p} u_{\max }\left(x_{1}, z\right)
$$


Se $x=\left(x_{1}, x^{\prime}\right) \in \partial D$ então $x_{1}=0 \operatorname{logo} w(x)=u_{\max }\left(0, x^{\prime}+t\right)=+\infty$. Mostrando que $w$ é solução de (2.56). Daí, segue da AfIRMAÇão 1 que $w(x) \leq u_{\max }(x)$, isto é $u_{\max }\left(x_{1}, x^{\prime}+t\right) \leq u_{\max }\left(x_{1}, x^{\prime}\right)$. Uma vez que $u_{\max }$ é $C^{1}(D)$,

$$
\lim _{s \rightarrow 0^{+}} \frac{u_{\max }\left(x_{1}, x^{\prime}+s t\right)-u_{\max }\left(x_{1}, x^{\prime}\right)}{s} \leq 0
$$

e

$$
\lim _{s \rightarrow 0^{-}} \frac{u_{\max }\left(x_{1}, x^{\prime}+s t\right)-u_{\max }\left(x_{1}, x^{\prime}\right)}{s} \geq 0
$$

temos $\frac{\partial u_{\max }\left(x_{1}, x^{\prime}\right)}{\partial x^{\prime}}=0$. Concluindo que $u_{\max }$ não depende de $x^{\prime}$. Logo $u_{\max }$ é solução para (2.31), segue do Lema (2.1.1), que $u_{\max }=u_{0}$.

Encontramos a solução mínima escolhendo a sequência de domínios $\left\{D_{k}^{\prime}\right\} \operatorname{com} D_{k}^{\prime} \subset$ $D_{k+1}^{\prime}$ e $D=\cup_{k=1}^{\infty} D_{k}^{\prime}$. Tome $D_{k}^{\prime}$ com a propriedade adicional $B_{3 k}(0) \cap \partial D \subset \partial D_{k}^{\prime} \cap \partial D$.

Fixe um inteiro positivo $k$ e escolha uma função suave $\psi_{k}$ com $0 \leq \psi_{k} \leq 1$, e $\psi_{k}=1$ em $\partial D_{k}^{\prime} \cap \partial D \cap B_{k}(0)$ e $\psi_{k}=0$ em $\partial D_{k}^{\prime} \backslash\left(B_{2 k}(0) \cap \partial D\right)$. O problema

$$
\left\{\begin{array}{lrrrr}
\triangle_{p} v & = & Q_{0} x_{1}^{-\gamma} v^{q}, & x \in D_{k}^{\prime}, \\
v & = & n \psi_{k}, & x \in \partial D_{k}^{\prime}
\end{array}\right.
$$

tem uma única solução positiva $v_{k, n}$ para todo $n$. Pois $\underline{v}=0$ é uma subsolução para (2.59)

$$
\begin{aligned}
\triangle_{p} \underline{v} & =0, \quad x \in D_{k}^{\prime}, \\
\underline{v} & =0 \leq n \psi_{k}, \quad x \in \partial D_{k}^{\prime}
\end{aligned}
$$

e $\bar{v}=n$ é supersolução para (2.59)

$$
\begin{aligned}
\triangle_{p} \bar{v} & =0 \leq Q_{0} x^{-1} n, \quad x \in D_{k}^{\prime} \\
\bar{v} & =n \geq n \psi_{k}
\end{aligned}
$$

logo existe uma solução para (2.59).

Por comparação, vamos provar que $v_{k, n}$ é crescente em $n$. Como

$$
\begin{aligned}
\triangle_{p} v_{k, n} & =Q_{0} x_{1}^{-\gamma} v_{k, n}^{q}, \\
\triangle_{p} v_{k, n+1} & =Q_{0} x_{1}^{-\gamma} v_{k, n+1}^{q}, \\
v_{k, n}(x)=n \psi_{k}(x) & \leq(n+1) \psi_{k}(x)=v_{k, n+1}(x)
\end{aligned}
$$


segue que $v_{k, n} \leq v_{k, n+1}$ em $D_{k}^{\prime}$, logo $v_{k, n}$ é crescente em $n$. Usando comparação, temos

$$
\begin{aligned}
\triangle_{p} v_{k, n} & \leq Q_{0} x_{1}^{-\gamma} v_{k, n}^{q} \\
\triangle_{p} u_{0} & \leq Q_{0} x_{1}^{-\gamma} u_{0}^{q} \\
v_{k, n}(x) & =n \psi_{k}(x) \leq \infty=u_{0}(x) \quad x \in \partial D_{k}^{\prime}
\end{aligned}
$$

$\operatorname{assim} v_{k, n} \leq u_{0}$ em $D_{k}^{\prime}$. Escolha $\psi_{k}$ tal que $\psi_{k} \leq \psi_{k+1}$ em $\partial D_{k}^{\prime} \cap \partial D_{k+1}^{\prime} \cap \partial D$ logo

$$
v_{k, n}(x)=n \psi_{k}(x) \leq n \psi_{k+1}(x)=v_{k+1, n}(x)
$$

em $\partial D_{k}^{\prime} \cap \partial D_{k+1}^{\prime} \cap \partial D$, então $v_{k, n}$ é crescente em $k$. Como a sequência é crescente em $k$ e limitada superiormente temos que $v_{k, n}$ converge em $C^{1}(D)$ para $v_{n}$ que é solução de

$$
\left\{\begin{array}{lrrrr}
\triangle_{p} v & = & Q_{0} x_{1}^{-\gamma} v^{q}, & x \in D \\
v & = & n, & x \in \partial D
\end{array}\right.
$$

quando $k \rightarrow \infty$. Como $\lim _{k \rightarrow \infty} \psi_{k}=1$ em $\partial D$. Portanto $v_{n} \leq u_{0}$ em $D$.

Como $v_{n}$ é crescente em $n$ e é limitada superiormente, temos que $v_{n}$ converge para $u_{\text {min }}$, que é solução de (2.56) em $C^{1}(D) \operatorname{com} n \rightarrow \infty$. Além disso, $u_{\text {min }}$ é solução minimal, pois se existe outra solução $u$ de (2.56), temos

$$
\begin{aligned}
\triangle_{p} v_{k, n} & =Q_{0} x_{1}^{-\gamma} v_{k, n}^{q}, \\
\triangle_{p} u & =Q_{0} x_{1}^{-\gamma} u^{q}, \\
v_{k, n}(x)=n \psi_{k} & <\infty=u(x) \text { na } \partial D_{k}^{\prime} .
\end{aligned}
$$

Pelo princípio da comparação

$$
v_{n, k}(x) \leq u(x), \quad \forall x \in D_{k}^{\prime}
$$

Tomando o limite em $k \rightarrow \infty$ e $n \rightarrow \infty$ temos

$$
u_{\min }(x) \leq u(x), \quad x \in D
$$


E $u_{\text {min }}$ depende apenas de $x_{1}$. De fato, seja $t \in \mathbb{R}^{n-1}$ arbitrário. Defina $w(x)=$ $u_{\min }\left(x_{1}, x^{\prime}+t\right)$

$$
\begin{aligned}
\nabla w(x) & =\left(\frac{\partial w(x)}{\partial x_{1}}, \frac{\partial w(x)}{\partial x_{2}}, \ldots, \frac{\partial w(x)}{\partial x_{n}}\right) \\
& =\left(\frac{u_{\min }\left(x_{1}, z\right)}{\partial x_{1}}, \frac{u_{\min }\left(x_{1}, z\right)}{\partial z_{1}}, \ldots, \frac{u_{\min }\left(x_{1}, z\right)}{\partial z_{n-1}}\right) \\
& =\nabla u_{\min }\left(x_{1}, z\right)
\end{aligned}
$$

onde $z=x^{\prime}+t \in \mathbb{R}^{n-1}$. Deste fato, temos:

$$
\begin{aligned}
\operatorname{div}\left(|\nabla w(x)|^{p-2} \nabla w(x)\right) & =\frac{\partial}{\partial x_{1}}\left(\frac{\partial u_{\min }\left(x_{1}, z\right)}{\partial x_{1}}\right) \\
& +\sum_{i=2}^{n} \frac{\partial}{\partial x_{i-1}}|\nabla w(x)|^{p-2} \frac{u_{\min }\left(x_{1}, z\right)}{\partial z_{i-1}} \\
& =\frac{\partial}{\partial x_{1}}\left(\frac{\partial u_{\min }\left(x_{1}, z\right)}{\partial x_{1}}\right) \\
& +\sum_{j=1}^{n-1} \frac{\partial}{\partial z_{i}}\left(|\nabla w(x)|^{p-2} \frac{u_{\min }\left(x_{1}, z\right)}{\partial z_{i}}\right) \\
& =\operatorname{div}\left(\left|\nabla u_{\min }\left(x_{1}, z\right)\right|^{p-2} \nabla u_{\min }\left(x_{1}, z\right)\right),
\end{aligned}
$$

ou seja,

$$
\triangle_{p} w=\triangle_{p} u_{\min }\left(x_{1}, z\right)
$$

Quando $x \in \partial D$ então $x_{1}=0 \mathrm{e}$

$$
w(x)=u_{\min }\left(0, x^{\prime}+t\right)=+\infty \quad \Rightarrow \quad w(x)=+\infty
$$

assim $w(x)$ é solução de (2.56) e pelo que foi concluido em $(2.61) u_{\min }(x) \leq w(x)$ então

$$
u_{\min }(x) \leq u_{\min }\left(x_{1}, x^{\prime}+t\right), \quad \forall x_{1}>0, x^{\prime} \in \mathbb{R}^{n-1} .
$$


Análogo ao que foi feito para a solução maximal, podemos concluir que para $t$ arbitrário, temos que $u_{\text {min }}$ depende apenas de $x_{1}$. Assim $u_{\text {min }}$ é solução de (2.31) e pelo Lema 2.1.1 obtemos $u_{\min }=u_{0}$.

Como a solução maximal e minimal de (2.56) coincidem, o problema tem uma única solução positiva que é $u_{0}$. Isto conclui a prova.

Nosso próximo resultado será necessário para provar a estimativa (2.3) do Teorema (2.1).

Lema 2.1.2 Seja u uma solução positiva para (2.1). Existe uma vizinhança $U$ de $\partial \Omega$ e constantes positivas $C_{1}, C_{2}$ tal que

$$
C_{1} d(x)^{-\alpha(x)} \leq u(x) \leq C_{2} d(x)^{-\alpha(x)}
$$

em $U$, onde $\alpha(x)=\frac{p-\gamma(x)}{q-p+1}$.

Prova: Fixe $x_{0} \in \partial \Omega$ e para $x \in \Omega$ introduza a função $v(y)=d(x)^{\alpha(x)} u(x+d(x) y)$, onde $y \in B_{1}(0)$. Então $v$ verifica

$$
\triangle_{p} v=d(x)^{\gamma(x)} a(x+d(x) y) v^{q} \quad \text { em } \quad B_{\frac{1}{2}}(0)
$$

já que

$$
\nabla v(y)=\left(\frac{\partial v}{\partial y_{1}}(y), \ldots, \frac{\partial v}{\partial y_{n}}(y)\right)
$$

e

$$
\frac{\partial v}{\partial y_{i}}(y)=d(x)^{\alpha(x)} \frac{\partial u}{\partial z_{i}}(z) \frac{\partial z_{i}}{\partial y_{i}}=d(x)^{\alpha(x)} \frac{\partial u}{\partial z_{i}}(z) d(x)=d(x)^{\alpha(x)+1} \frac{\partial u}{\partial z_{i}}(z)
$$

onde $z=x+d(x) y$, então

$$
\nabla v(y)=d(x)^{\alpha(x)+1}\left(\frac{\partial u}{\partial z_{1}}(z), \ldots, \frac{\partial u}{\partial z_{n}}(z)\right)=d(x)^{\alpha(x)+1} \nabla u(x+d(x) y)
$$


assim

$$
\begin{aligned}
|\nabla v(y)|^{p-2} \nabla v(y) & =d(x)^{(\alpha(x)+1)(p-2)} d(x)^{(\alpha(x)+1)}|\nabla u(x+d(x) y)|^{p-2} \nabla u(x+d(x) y) \\
& =d(x)^{(\alpha(x)+1)(p-1)}|\nabla u(x+d(x) y)|^{p-2} \nabla u(x+d(x) y) \\
& =d(x)^{(\alpha(x)+1)(p-1)}|\nabla u(z)|^{p-2} \nabla u(z)
\end{aligned}
$$

segue que

$$
\begin{aligned}
\operatorname{div}\left(|\nabla v(y)|^{p-2} \nabla v(y)\right) & =\sum_{i=1}^{n} \frac{\partial}{\partial y_{i}}\left(d(x)^{(\alpha(x)+1)(p-1)}|\nabla u(z)|^{p-2} \frac{\partial u}{\partial z_{i}}(z)\right) \\
& =\sum_{i=1}^{n} d(x)^{(\alpha(x)+1)(p-1)} \frac{\partial}{\partial y_{i}}\left(|\nabla u(z)|^{p-2} \frac{\partial u}{\partial z_{i}}(z)\right) \\
& =\sum_{i=1}^{n} d(x)^{(\alpha(x)+1)(p-1)} \frac{\partial}{\partial z_{i}}\left(|\nabla u(z)|^{p-2} \frac{\partial u}{\partial z_{i}}(z)\right) \frac{\partial z_{i}}{\partial y_{i}} \\
& =\sum_{i=1}^{n} d(x)^{(\alpha(x)+1)(p-1)} \frac{\partial}{\partial z_{i}}\left(|\nabla u(z)|^{p-2} \frac{\partial u}{\partial z_{i}}(z)\right) d(x) \\
& =d(x)^{(\alpha(x)+1)(p-1)+1} \sum_{i=1}^{n} \frac{\partial}{\partial z_{i}}\left(|\nabla u(z)|^{p-2} \frac{\partial u}{\partial z_{i}}(z)\right) \\
& =d(x)^{(\alpha(x)+1)(p-1)+1} \triangle_{p} u(x+d(x) y) \\
& =d(x)^{\gamma(x)+\alpha q} a(x+d(x) y) u^{q}(x+d(x) y) \\
& =d(x)^{\gamma(x)} a(x+d(x) y) v^{q}(y) \\
& d(x) a(x+d(x) y)\left[d(x)^{\alpha} u(x+d(x) y)\right]^{q} \\
&
\end{aligned}
$$

onde $y \in B_{\frac{1}{2}}(0)$. Logo

$$
\triangle_{p} v=d(x)^{\gamma(x)} a(x+d(x) y) v^{q}(y) \quad \text { em } \quad B_{\frac{1}{2}}(0) .
$$


Como

$$
\lim _{x \rightarrow x_{0}} d(x)^{\gamma(x)} a(x)=Q\left(x_{0}\right)
$$

para todo $\varepsilon>0$ existe $\delta>0$ tal que $\left\|x-x_{0}\right\|<\delta$ então

$$
\begin{gathered}
\left|d(x)^{\gamma(x)} a(x)-Q\left(x_{0}\right)\right|<\varepsilon \quad \Rightarrow \quad-\varepsilon+Q\left(x_{0}\right)<d(x)^{\gamma(x)} a(x)<\varepsilon+Q\left(x_{0}\right) \\
\Rightarrow \quad C_{1}<d(x)^{\gamma(x)} a(x) \quad<C_{2}
\end{gathered}
$$

para $x$ próximo de $x_{0}$. Uma vez que $d(x+d(x) y)<d(x)$, temos

$$
\begin{aligned}
a(x) \geq C_{1} d(x)^{-\gamma(x)} & \Rightarrow a(x+d(x) y) \geq C_{1} d(x+d(x) y)^{-\gamma(x+d(x) y)} \\
\Rightarrow a(x+d(x) y) & \geq C_{1} d(x)^{-\gamma(x+d(x) y)}
\end{aligned}
$$

para $x$ próximo de $x_{0}$. Deste modo, temos

$$
\triangle_{p} v=d(x)^{\gamma(x)} a(x+d(x) y) v^{q} \geq C_{1} d(x)^{\gamma(x)-\gamma(x+d(x) y)} v^{q}
$$

em $B_{\frac{1}{2}}(0)$. Por outro lado, desde que $\gamma \in C^{\mu}(\bar{\Omega})$, temos

$$
|\gamma(x)-\gamma(x+d(x) y)| \leq C d(x)^{\mu} \quad \Rightarrow
$$

ou seja,

$$
-C d^{\mu} \leq \gamma(x)-\gamma(x+d(x) y) \leq C d^{\mu}
$$

De (2.64) e (2.65)

$$
\triangle_{p} v \geq C_{1} d(x)^{\gamma(x)-\gamma(x+d(x) y)} v^{q} \geq C_{1} d(x)^{C d(x)^{\mu}} v^{q}
$$

já que $d(x)$ é suficientemente pequeno.

Agora, observando que

$$
\lim _{d(x) \rightarrow 0} d(x)^{C d(x)^{\mu}}=\lim _{d(x) \rightarrow 0} e^{\ln d(x)^{C d(x)^{\mu}}}=\lim _{d(x) \rightarrow 0} e^{C d(x)^{\mu} \ln d(x)}
$$


e

$\lim _{d(x) \rightarrow 0} C d(x)^{\mu} \ln d(x)=C \lim _{d(x) \rightarrow 0} \frac{\ln d(x)}{d(x)^{-\mu}}=C \lim _{d(x) \rightarrow 0} \frac{1}{-\mu d(x)^{-\mu}}=\frac{-C}{\mu} \lim _{d(x) \rightarrow 0} \frac{1}{d(x)^{-\mu}}=0$,

temos

$$
\lim _{d(x) \rightarrow 0} d(x)^{C d(x)}=1
$$

De $(2.66)$ e (2.67) temos

$$
\triangle_{p} v \geq C_{1} v^{q} \quad \text { em } \quad B_{\frac{1}{2}}(0) .
$$

Seja $U \in W_{l o c}^{1, p}\left(B_{\frac{1}{2}}(0)\right) \cap C^{1, \alpha}\left(B_{\frac{1}{2}}(0)\right), \quad 0<\alpha<1$ a solução não-negativa de

$$
\left\{\begin{array}{l}
\triangle_{p} U=C U^{q}, \quad x \in B_{\frac{1}{2}}(0), \\
U=+\infty, \quad x \in \partial B_{\frac{1}{2}}(0),
\end{array}\right.
$$

a solução de (2.68) é garantida pelo Lema (F.1) no Anexo 1, onde a função $U^{q}$ satisfaz a condição de Keller-Osserman pelo Apêndice A. Como

$$
\begin{aligned}
-\triangle_{p} v & \leq-C_{1} v^{q} \\
-\triangle_{p} U & =-C_{1} U^{q} \\
v(y) \leq U(y) & =+\infty, \quad \text { na } \partial B_{\frac{1}{2}}(0),
\end{aligned}
$$

pelo princípio da comparação $v \leq U$ em $B_{\frac{1}{2}}(0)$. Tomando $y=0$

$$
v(0) \leq U(0) \quad \Rightarrow \quad d(x)^{\alpha(x)} u(x) \leq U(0) \quad \Rightarrow \quad u(x) \leq d(x)^{-\alpha(x)} U(0) .
$$

AfirmaÇão $1 U(0)>0$

Note que

- $\beta(s)=s^{q}$ é não-decrescente,

- $\beta(0)=0$ e

- $\beta(s)>0$ para todo $s>0$.

Como $U(x)=+\infty$ na $\partial B_{\frac{1}{2}}(0)$, temos $U(x) \neq 0$ em algum ponto de $B_{\frac{1}{2}}(0)$ então pelo Teorema (1.6.1) temos $U(x)>0$ em $B_{\frac{1}{2}}(0)$, assim $U(0)>0$. Provamos, assim o lado direito de (2.62) para $x$ próximo da fronteira $\partial \Omega$. 
Para provarmos o lado esquerdo de (2.62) seja $x_{0} \in \partial \Omega$ fixado. Para um ponto $x \in \Omega$ perto de $x_{0}$ denote por $\bar{x}$ sua projeção sobre $\partial \Omega$. Denotamos $c_{x}=\bar{x}+d(x) \nu(\bar{x})$ onde $\nu(\bar{x})$ é o vetor unitário normal exterior a $\partial \Omega$ em $\bar{x}$. Observe que $c_{x} \notin \Omega$ para $x$ perto de $x_{0}$.

Considere o anel $A_{x}=\{y \in \Omega ; d(x)<d(y)<2 d(x)\}$. Defina

$$
w(y)=d(x)^{\alpha(x)} u\left(c_{x}+d(x) y\right)
$$

onde $y \in Q_{x}=\left\{y \in A_{\text {anel }} ; c_{x}+d(x) y \in A_{x}\right\}$ onde $A_{\text {anel }}=\left\{y \in \mathbb{R}^{n} ; 2 \leq|y|<3\right\}$ é o anel normalizado. Então $w$ verifica

$$
\triangle_{p} w=d(x)^{\gamma(x)} a\left(c_{x}+d(x) y\right) w^{q} \quad \text { em } \quad Q_{x}
$$

Pois

$$
\frac{\partial w}{\partial y_{i}}(y)=d(x)^{\alpha(x)} \frac{\partial}{\partial y_{i}} u\left(c_{x}+d(x) y\right)=d(x)^{\alpha(x)} \frac{\partial u}{\partial z_{i}}(z) d(x)=d(x)^{\alpha(x)+1} \frac{\partial u}{\partial z_{i}}(z)
$$

onde $z=c_{x}+d(x) y$. Assim

$$
\begin{aligned}
\nabla w & =d(x)^{\alpha(x)+1}\left(\frac{\partial u}{\partial z_{1}}(z), \ldots, \frac{\partial u}{\partial z_{n}}(z)\right)=d(x)^{\alpha(x)+1} \nabla u(z) \Rightarrow \\
|\nabla w|^{p-2} \nabla w & =d(x)^{(\alpha(x)+1)(p-1)}|\nabla u(z)|^{p-2} \nabla u(z)
\end{aligned}
$$

Agora

$$
\begin{aligned}
\triangle_{p} w & =d i v\left(|\nabla w|^{p-2} \nabla w\right)=d(x)^{(\alpha(x)+1)(p-1)} \sum_{i=1}^{n} \frac{\partial}{\partial y_{i}}\left(|\nabla u(z)|^{p-2} \frac{\partial u}{\partial z_{i}}(z)\right) \\
& =d(x)^{(\alpha(x)+1)(p-1)+1} \sum_{i=1}^{n} \frac{\partial}{\partial z_{i}}\left(|\nabla u(z)|^{p-2} \frac{\partial u}{\partial z_{i}}(z)\right) \\
& =d(x)^{\gamma(x)+\alpha(x) q} \triangle_{p} u\left(c_{x}+d(x) y\right) \\
& =d(x)^{\gamma(x)+\alpha(x) q} a\left(c_{x}+d(x) y\right) u^{q}\left(c_{x}+d(x) y\right) \\
& =d(x)^{\gamma(x)} a\left(c_{x}+d(x) y\right)\left[d(x)^{\alpha(x)} u\left(c_{x}+d(x) y\right)\right]^{q} \\
& =d(x)^{\gamma(x)} a\left(c_{x}+d(x) y\right) w(y)^{q}
\end{aligned}
$$

em $Q_{x}$. Usando a desigualdade a direita de (2.63), temos

$$
d(x)^{\gamma(x)} a(x) \leq C_{2} \quad \text { quando } x \text { próximo de } x_{0}
$$


temos

$$
a(x) \leq C_{2} d(x)^{-\gamma(x)} \Rightarrow a\left(c_{x}+d(x) y\right) \leq C_{2} d\left(c_{x}+d(x) y\right)^{-\gamma\left(c_{x}+d(x) y\right)} .
$$

Como

$$
d(x) \leq d\left(c_{x}+d(x) y\right) \leq 2 d(x) \quad \text { para } y \in Q_{x} .
$$

Assim

$$
\begin{aligned}
\triangle_{p} w & =d(x)^{\gamma(x)} a\left(c_{x}+d(x) y\right) w(y)^{q} \leq C_{2} d(x)^{\gamma(x)} d\left(c_{x}+d(x) y\right)^{-\gamma\left(c_{x}+d(x) y\right)} w(y)^{q} \\
& \leq \widetilde{C} d(x)^{\gamma(x)-\gamma\left(c_{x}+d(x) y\right)} w^{q}(y)
\end{aligned}
$$

Como $\gamma \in C^{\mu}(\bar{\Omega})$ é Holder contínua,

$\left|\gamma(x)-\gamma\left(c_{x}+d(x) y\right)\right|<C\left|x-c_{x}-d(x) y\right|^{\mu}=C|x-\bar{x}-d(x) \nu(\bar{x})-d(x) y|^{\mu} \leq C_{1} d(x)^{\mu}$

então se $d(x)$ é suficientemente pequeno, temos

$$
\triangle_{p} w \leq C w^{q}(y) \quad \text { em } Q_{x}
$$

Por outro lado, o problema

$$
\left\{\begin{array}{lll}
\triangle_{p} z=C z^{q} & \text { em } & A_{\text {anel }} \\
z=\eta & \text { em } & |y|=1 \\
z=0 & \text { em } & |y|=3
\end{array}\right.
$$

onde $\eta>0$ uma constante qualquer, admite uma solução $z$, pois $\underline{z}=0$ é uma subsolução e $\bar{z}=\eta$ é supersolução do problema (2.69). Pela desigualdade de Harnack temos que $0<z \leq \eta$ em $A_{\text {anel }}$. Por Diaz [2] z é radialmente simétrica. Por comparação, como

$$
\begin{aligned}
-\triangle_{p} z & =-C z^{q} \text { em } Q_{x} \\
-\triangle_{p} w & \geq-C w^{q} \text { em } Q_{x} \\
w & \geq 0 \text { em }\left\{y \in \partial Q_{x} ;|y|=3\right\} \\
z & \leq \eta \leq w(y)=+\infty \text { em }\left\{y \in \partial Q_{x} ; 1 \leq|y| \leq 3\right\}
\end{aligned}
$$

temos $w \geq z$ em $Q_{x}$. Como $-2 \nu(\bar{x}) \in Q_{x}$, pois $-2 \nu(\bar{x}) \in A_{\text {anel }}$ já que $|-2 \nu(\bar{x})|=2 \mathrm{e}$

$$
c_{x}+d(x)(-2 \nu(\bar{x}))=\bar{x}+d(x) \nu(\bar{x})-2 d(x) \nu(\bar{x})=\bar{x}-d(x) \nu(\bar{x})=x \in \Omega .
$$


Assim

$$
\begin{array}{rll}
w(-2 \nu(\bar{x})) & \geq z(-2 \nu(\bar{x})) & \Rightarrow \\
d(x)^{\alpha(x)} u\left(c_{x}+d(x)(-2 \nu(\bar{x}))\right) & \geq z(-2 \nu(\bar{x})) & \stackrel{2.70}{\Rightarrow} \\
d(x)^{\alpha(x)} u(x) & \geq z(-2 \nu(\bar{x})) \quad \Rightarrow \\
u(x) & \geq d(x)^{-\alpha(x)} z(-2 \nu(\bar{x})),
\end{array}
$$

como $z$ é radialmente simétrica temos, $z(-2 \nu(\bar{x}))=z(2)>0$. Provamos, assim, o lado esquerdo de (2.62).

Portanto, para $x$ próximo de $x_{0}$

$$
\widetilde{C} d(x)^{-\alpha(x)} \leq u(x) \leq C d(x)^{-\alpha(x)}
$$

por um argumento de compacidade podemos concluir a desigualdade é válida em uma vizinhança de $\partial \Omega$.

\subsection{Prova da Existência}

Nesta seção iremos provar a existência de solução para o problema (2.1).

Considere o problema

$$
\left\{\begin{array}{l}
\triangle_{p} u=a(x) u^{q}, \quad x \in \Omega, \\
u \quad=n, \quad x \in \partial \Omega
\end{array}\right.
$$

para $n$ inteiro positivo. Como a função $a$ é singular na $\partial \Omega$, para obter uma solução vamos truncar a função peso $a(x)$ como segue: escolha uma função suave $\psi(t)$ não decrescente tal que $0 \leq \psi \leq 1$, com $\psi=0$ em $[0,1]$ e $\psi=1$ em $[2,+\infty)$ e para um inteiro positivo $k$ seja $a_{k}(x)=\psi(k d(x)) a(x)$. Como $a$ é não negativa a sequência $\left\{a_{k}\right\}$ é não decrescente em $k$,

$$
\begin{aligned}
a_{k}(x) & =\psi(k d(x)) a(x), \\
a_{k+1}(x) & =\psi((k+1) d(x)) a(x)=\psi(k d(x)+d(x)) a(x) .
\end{aligned}
$$

Como fizemos uma translação da função $\psi$ a esquerda, temos $\psi(k d(x)+d(x)) \geq \psi(k d(x))$ $\operatorname{logo} a_{k+1}(x) \geq a_{k}(x)$ e $a_{k}(x) \leq a(x) \leq C d(x)^{-\gamma(x)}$. Pois $\lim _{x \rightarrow x_{0}} d(x)^{\gamma(x)} a(x)=Q\left(x_{0}\right)$ com 
$x_{0} \in \partial \Omega, \gamma(x)<p$ na $\partial \Omega$ então

$$
\begin{array}{r}
\forall \varepsilon>0 \quad \exists \delta>0 \quad \text { tal que se }\left|x-x_{0}\right|<\delta \text { então } \\
\left|d(x)^{\gamma(x)} a(x)-Q\left(x_{0}\right)\right|<\varepsilon \Rightarrow \varepsilon<d(x)^{\gamma(x)} a(x)-Q\left(x_{0}\right)<\varepsilon \Rightarrow \\
Q\left(x_{0}\right)-\varepsilon<d(x)^{\gamma(x)} a(x)<Q\left(x_{0}\right)+\varepsilon \Rightarrow C_{1}<d(x)^{\gamma(x)} a(x)<C_{2} \Rightarrow \\
d(x)^{-\gamma(x)} C_{1}<a(x)<d(x)^{-\gamma(x)} C_{2}, \quad \text { em }\left|x-x_{0}\right|<\delta .
\end{array}
$$

E em $\Omega_{\delta}=\Omega \backslash\left\{x \in \mathbb{R}^{n} ;\left|x-x_{0}\right|<\delta\right\}$ temos $\Omega_{\delta}$ é compacto e a função $g(x)=$ $d(x)^{\gamma(x)} a(x)$ é contínua, portanto limitada logo $C_{1} \leq d(x)^{\gamma(x)} a(x) \leq C_{2}$. Assim $C_{1} d(x)^{-\gamma(x)} \leq$ $a(x) \leq C_{2} d(x)^{-\gamma(x)}$ em $\Omega_{\delta}$. Então para $x \in \Omega$ temos

$$
C_{1} d(x)^{-\gamma(x)} \leq a(x) \leq C_{2} d(x)^{-\gamma(x)}
$$

E além disso $a_{k} \in L^{\infty}(\Omega)$ pois, quando $d(x)<\frac{1}{k}$ temos $\psi(k d(x))=0$ e $a_{k}(x)=0$ e para $x \in \Omega-\left\{x ; d(x)<\frac{1}{k}\right\}$ temos que $a_{k}$ é contínua em compacto, logo é limitada.

Considere o problema truncado

$$
\begin{cases}\triangle_{p} u & =a_{k}(x) u^{q}, \quad x \in \Omega \\ u & =n, \quad x \in \partial \Omega\end{cases}
$$

$\underline{u}=0$ é uma subsolução do problema (2.73) pois

$$
\begin{gathered}
\triangle_{p} \underline{u}=0=a_{k}(x) 0, \\
\underline{u}=0<n, \text { na } \partial \Omega
\end{gathered}
$$

e $\bar{u}=n$ é uma supersolução do problema (2.73) pois

$$
\begin{aligned}
\triangle_{p} \bar{u} & =0 \leq a_{k}(x) n^{q} \quad \Rightarrow \quad-\triangle_{p} \bar{u} \geq-a_{k}(x) n^{q} \\
\bar{u} & =n, \text { na } \partial \Omega .
\end{aligned}
$$

Então o problema (2.73) tem uma solução não negativa. E essa é única pelo princípio da comparação, pois se $u$ e $w$ são soluções de (2.73)

$$
\begin{array}{r}
\triangle_{p} u=a_{k}(x) u^{q}, \\
\triangle_{p} w=a_{k}(x) w^{q}, \\
u=w=n, \text { na } \partial \Omega,
\end{array}
$$

então aplicando o princípio da comparação duas vezes, obtemos, $u=w$. Denotamos tal 
solução por $u_{k, n}$.

Nosso próximo passo é mostrar que podemos passar o limite com $k \rightarrow+\infty$. É neste passo que a condição $\gamma(x)<p$ é essencial. Defina $u_{k, n}=v_{k, n}+n$, então $v_{k, n}$ é solução de

$$
\left\{\begin{array}{l}
\triangle_{p} v=a_{k}(x)\left(v_{k, n}+n\right)^{q} \quad x \in \Omega, \\
v=0 \quad x \in \partial \Omega
\end{array}\right.
$$

pois

$$
\triangle_{p} v_{k, n}=\triangle_{p} u_{k, n}=a_{k}(x)\left(v_{k, n}+n\right)^{q} \quad \text { em } \Omega
$$

e na $\partial \Omega$ temos $u_{k, n}=n \Rightarrow v_{k, n}+n=n \Rightarrow v_{k, n}=0$.

Observe que o lado direito de (2.74) é limitado por $C_{2} n^{q} d(x)^{-\gamma(x)} \operatorname{com} \gamma(x)<p$ na $\partial \Omega$, pois usando (2.72) temos

$$
\triangle_{p} v_{k, n}=a_{k}(x)\left(v_{k, n}+n\right)^{q} \leq C_{2} d(x)^{-\gamma(x)}\left(v_{k, n}+n\right)^{q} \leq C_{2} d(x)^{-\gamma(x)} n^{q}
$$

já que $\bar{u}=n$ é supersolução de $(2.73)$ então $u_{k, n} \leq n$ então

$$
u_{k, n}^{q} \leq n^{q} \quad \Rightarrow \quad\left(v_{k, n}+n\right)^{q} \leq n^{q}
$$

Seja $\gamma_{0} \in(0, p)$, e seja $\phi$ a única solução para

$$
\begin{cases}-\triangle_{p} \phi & =d^{-\gamma_{0}}, \quad x \in \Omega \\ \phi & =0 \quad x \in \partial \Omega\end{cases}
$$

a existência da solução de (2.75) é garantida pelo Teorema (2.1.1), e essa solução é positiva pois, já que $\underline{\phi}=0$ é uma subsolução

$$
\begin{array}{r}
-\triangle_{p} \underline{\phi}=0 \leq d^{-\gamma_{0}} \\
\underline{\phi}=0
\end{array}
$$

então $\phi \geq 0$ e pelo Teorema 5 de [19] temos que $\phi>0$. Como

$$
-\triangle_{p}\left(-\left(C n^{q}\right)^{\frac{1}{p-1}} \phi(x)\right)=-C n^{q}\left(-\triangle_{p} \phi(x)\right)=-C n^{q} d(x)^{-\gamma_{0}}, \quad \text { em } \Omega
$$

e vimos que $\triangle_{p} v_{k, n} \leq C_{2} d(x)^{-\gamma(x)} n^{q}$ então

$$
-\triangle_{p} v_{k, n} \geq-C_{2} d(x)^{-\gamma(x)} n^{q}
$$


para $\gamma(x)<p$ na $\partial \Omega$.

AfirmaÇ̃̃o $1-\triangle_{p} v_{k, n} \geq-C d(x)^{-\gamma_{0}} n^{q}$ em $\Omega$

Para $x$ numa vizinhança $V$ de $\partial \Omega$ tal que $d(x)$ é suficientemente pequeno, $d(x)<1$, como $\gamma(x)<\gamma_{0}<p$ então $-\gamma_{0}<-\gamma(x)$ assim

$$
\begin{aligned}
d(x)^{-\gamma_{0}} & \geq d(x)^{-\gamma(x)} \Rightarrow-d(x)^{-\gamma_{0}} \leq-d(x)^{-\gamma(x)} \\
-\triangle_{p} v_{k, n} & \geq-C_{2} d(x)^{-\gamma(x)} n^{q} \geq-C_{2} d(x)^{-\gamma_{0}} n^{q} .
\end{aligned}
$$

Quando $x$ em $\Omega-V$ temos que o conjunto $\Omega-V$ é compacto e a função $d(x)^{-\gamma(x)}$ é contínua em um conjunto compacto então é limitada, e $d(x)^{-\gamma_{0}}$ também é limitada nesse conjunto, e $d(x)^{-\gamma_{0}}>0$, assim existem $\tilde{C}, \bar{C}$, constantes positivas tais que

$$
d(x)^{-\gamma(x)} \leq \bar{C} \text { e } d(x)^{-\gamma_{0}} \geq \tilde{C}>0
$$

então

$$
\begin{aligned}
& -d(x)^{-\gamma(x)} C_{2} n^{q} \geq-C_{3} n^{q}, \text { onde } C_{3}=\bar{C} C_{2}, \\
& -d(x)^{-\gamma_{0}} \leq-\tilde{C} \Rightarrow-\frac{C_{3}}{\tilde{C}} d(x)^{-\gamma_{0}} n^{q} \leq-\frac{C_{3}}{\tilde{C}} \tilde{C} n^{q}=-C_{3} n^{q} \Rightarrow \\
& -C_{4} d(x)^{-\gamma_{0}} n^{q} \leq-C_{3} n^{q}, \quad \text { onde } C_{4}=\frac{C_{3}}{\tilde{C}} \\
& -\triangle_{p} v_{k, n} \geq-C_{2} d(x)^{-\gamma(x)} n^{q} \geq-C_{3} n^{q} \geq-C_{4} d(x)^{-\gamma_{0}} n^{q}
\end{aligned}
$$

tomando $C=\max \left\{C_{2}, C_{4}\right\}$ temos $-\triangle_{p} v_{k, n} \geq-C d(x)^{-\gamma_{0}} n^{q}$ em $\Omega-V$ e $-\triangle_{p} v_{k, n} \geq$ $-C d(x)^{-\gamma_{0}} n^{q}$ em $V$ provando assim a AfirmaÇão 1.

$\mathrm{Na}$ fronteira de $\Omega$ temos

$$
v_{k, n}=0=-\left(C n^{q}\right)^{\frac{1}{p-1}} \phi(x) .
$$

Por comparação

$$
\begin{gathered}
v_{k, n} \geq-\left(C n^{q}\right)^{\frac{1}{p-1}} \phi(x), \quad \text { em } \Omega . \\
\mathrm{E}-\triangle_{p}\left(\left(C n^{q}\right)^{\frac{1}{p-1}} \phi(x)\right)=C n^{q}\left(-\triangle_{p} \phi(x)\right)=C n^{q} d(x)^{-\gamma_{0}}, \text { em } \Omega, \mathrm{e} \\
-\triangle_{p} v_{k, n}=-a_{k}\left(v_{k, n}+n\right)^{q} \leq 0 \leq C n^{q} d(x)^{-\gamma_{0}}
\end{gathered}
$$

e na fronteira de $\Omega$, temos

$$
v_{k, n}(x)=0=\left(C n^{q}\right)^{\frac{1}{p-1}} \phi(x), \text { em } \partial \Omega
$$

então por comparação 


$$
v_{k, n}(x) \leq\left(C n^{q}\right)^{\frac{1}{p-1}} \phi(x), \quad \text { em } \Omega
$$

Então de (2.77) e (2.78) podemos concluir $\left|v_{k, n}(x)\right| \leq\left(C n^{q}\right)^{\frac{1}{p-1}} \phi(x)$ em $\Omega$. Isto nos dá uma limitação local para a sequência $v_{k, n}$. Argumentando como na prova do Teorema (2.1.1) (usando o processo diagonal). Obtemos $v_{k, n} \stackrel{k \rightarrow \infty}{\longrightarrow} v_{n} \mathrm{em} C^{1}(\Omega)$. Como

$$
\left|v_{k, n}\right| \leq\left(C n^{q}\right)^{\frac{1}{p-1}} \phi(x) \quad \Rightarrow \quad\left|v_{n}\right| \leq\left(C n^{q}\right)^{\frac{1}{p-1}} \phi(x)
$$

Se $x_{1} \in \partial \Omega$ existe $\left(x_{j}\right) \subset \Omega$ tal que $\lim _{j \rightarrow \infty} x_{j}=x_{1}$

$$
\begin{aligned}
& \left|v_{n}\left(x_{j}\right)\right| \leq\left(C n^{q}\right)^{\frac{1}{p-1}} \phi\left(x_{j}\right) \Rightarrow \\
& \left|v_{n}\left(x_{1}\right)\right|=\lim _{j \rightarrow \infty}\left|v_{n}\left(x_{j}\right)\right| \leq\left(C n^{q}\right)^{\frac{1}{p-1}} \phi\left(x_{1}\right)=0 \quad \forall x_{1} \in \partial \Omega .
\end{aligned}
$$

Como

$$
\begin{aligned}
\lim _{k \rightarrow \infty} a_{k}(x) & =\lim _{k \rightarrow \infty} \psi(k d(x)) a(x)=a(x) \lim _{k \rightarrow \infty} \psi(k d(x)) \\
& =1 a(x)=a(x) .
\end{aligned}
$$

Passando o limite com $k \rightarrow \infty$ em (2.74) temos que $v_{n}$ é solução de

$$
\begin{cases}\triangle_{p} v & =a(x)(v+n)^{q} \quad x \in \Omega \\ v & =0 \quad x \in \partial \Omega\end{cases}
$$

onde $u_{n}=v_{n}+n$ é uma solução não negativa de (2.71), pois

$$
\nabla u_{n}=\nabla v_{n} \quad \Rightarrow \quad \triangle_{p} u_{n}=\triangle_{p} v_{n}
$$

E na fronteira $\partial \Omega$, temos

$$
v_{n}=0 \quad \Rightarrow \quad u_{n}=n .
$$

A unicidade de $u_{n}$ é obtida pelo princípio da comparação, pois se $u_{n}$ e $w_{n}$ são soluções de (2.71) temos

$$
\begin{aligned}
-\triangle_{p} u_{n} & =-a(x) u_{n}^{q}, \\
-\triangle_{p} w_{n} & =-a(x) w_{n}^{q}, \\
u_{n}=n & =w_{n}, \quad \text { na } \partial \Omega
\end{aligned}
$$


usando comparação duas vezes, obtemos

$$
u_{n}=w_{n}
$$

A sequência $\left\{u_{n}\right\}$ é crescente, pois a solução do problema

$$
\left\{\begin{array}{l}
\triangle_{p} u=a_{k}(x) u^{q}, \quad x \in \Omega \\
u \quad=n, \quad x \in \partial \Omega
\end{array}\right.
$$

é $u_{k, n}$ e se $n<m$ então $u_{k, n}$ é subsolução e $m$ é supersolução de

$$
\begin{cases}\triangle_{p} u & =a_{k}(x) u^{q}, \quad x \in \Omega \\ u & =m, \quad x \in \partial \Omega\end{cases}
$$

Assim $u_{k, n} \leq u_{k, m} \leq m$, tomando $k \rightarrow \infty$ temos $u_{n} \leq u_{m}$. Agora temos que obter uma limitação uniforme local para a solução $u_{n}$. Como $a>0$ numa vizinhança de $\partial \Omega$, por hipótese. Escolhemos $\delta>0$ tal que $a>0$ em $\Omega_{\delta}=\{x \in \Omega ; d(x)<\delta\}$. Escolha $\varepsilon<\delta$ e um ponto $x_{0} \in \Omega_{\delta} \operatorname{com} d\left(x_{0}\right)=\frac{\varepsilon}{2}$. Uma vez que $a(x) \geq a_{0}>0$ em $B\left(x_{0}, \frac{\varepsilon}{4}\right)$, temos

$$
\triangle_{p} u_{n}=a(x) u_{n}^{q} \geq a_{0} u_{n}^{q} \quad \text { na } B\left(x_{0}, \frac{\varepsilon}{4}\right)
$$

Seja

$$
\left\{\begin{aligned}
\triangle_{p} U & =a_{0} U^{q}, \quad x \in B\left(x_{0}, \frac{\varepsilon}{4}\right), \\
U & =+\infty, \quad x \in \partial B\left(x_{0}, \frac{\varepsilon}{4}\right) .
\end{aligned}\right.
$$

O problema (2.82), tem solução pois $f(u)=u^{q}$ satisfaz as condições do Lema 2.1 de [13], então existe uma solução positiva $U$. Por comparação

$$
\begin{aligned}
-\triangle_{p} U & =-a_{0} U^{q}, \\
-\triangle_{p} u_{n} & \leq-a_{0} u_{n}^{q} \\
u_{n} & \leq \infty, \quad \text { na } \partial B\left(x_{0}, \frac{\varepsilon}{4}\right)
\end{aligned}
$$

temos $u_{n} \leq U$ em $B\left(x_{0}, \frac{\varepsilon}{4}\right)$. Isto mostra que $u_{n}$ é uniformemente limitada na $B\left(x_{0}, \frac{\varepsilon}{8}\right)$. Assim, obtemos que $u_{n}$ é uniformemente limitado no conjunto $\left\{x \in \Omega ; d(x)=\frac{\varepsilon}{2}\right\}$, isto é existe $c>0$ tal que $u_{n} \leq c$ em $\left\{x \in \Omega ; d(x)=\frac{\varepsilon}{2}\right\}$. E

$$
\begin{aligned}
& \triangle_{p} u_{n} \geq 0 \\
& \text { em } \Omega \backslash\left\{x \in \Omega ; d(x)<\frac{\varepsilon}{2}\right\} \\
& \triangle_{p} c=0 \text {, } \\
& \Omega \backslash\left\{x \in \Omega ; d(x)<\frac{\varepsilon}{2}\right\} \\
& u_{n} \leq c, \quad \partial\left(\Omega \backslash\left\{x \in \Omega ; d(x)=\frac{\varepsilon}{2}\right\}\right)=\left\{x \in \Omega ; d(x)=\frac{\varepsilon}{2}\right\}
\end{aligned}
$$


por comparação

$$
u_{n} \leq c \quad \text { em } \Omega \backslash\left\{x \in \Omega ; d(x)<\frac{\varepsilon}{2}\right\} .
$$

Como $\varepsilon$ é qualquer, tomando $\varepsilon \rightarrow 0$ e pelo o argumento de processo diagonal temos que uma subsequência de $u_{n}$ converge para $u$ em $C^{1}(\Omega)$, e $u$ é solução não negativa de (2.1). A solução $u$ de (2.1) é positiva pois $u(x)=+\infty$ na $\partial \Omega$ então existe uma vizinhança $V$ da fronteira de $\Omega$ tal que $u>0$ e seja o conjunto $\Omega-V$, este conjunto é compacto e a função $a(x)$ é contínua e não negativa em $\Omega$ então $a(x)$ é limitada assim

$$
\triangle_{p} u=a(x) u^{q} \leq C u^{q}=\beta(u)
$$

onde $\beta:[0,+\infty] \rightarrow \mathbb{R}$ é contínua, não decrescente e $\beta(0)=0$ então pelo Teorema 5 de [19] temos $u>0$ em $\Omega-V$. Assim $u$ é positiva em $\Omega$.

\subsection{Estimativa (2.3) do Teorema (2.1)}

Seja $x_{0} \in \partial \Omega$. Sem perda de generalidade podemos assumir que $x_{0}=0$ e o vetor normal unitário exterior $\nu\left(x_{0}\right)=-e_{1}$, onde $e_{1}$ é o primeiro vetor da base canônica de $R^{n}$. Tomando $x_{n}$ uma sequência em $\Omega$ tal que $x_{n} \rightarrow 0$. Para cada $x_{n}$ existe $d_{n}>0$ tal que $x_{n}+d_{n}\left(-e_{1}\right)=\xi_{n} \in \partial \Omega$. Seja $z_{n}=\xi_{n}+d_{n}\left(-\nu\left(\xi_{n}\right)\right)$. Note que $\xi_{n}$ é projeção de $z_{n}$ em $\partial \Omega$, se $x_{n} \rightarrow x_{0}$ então $z_{n} \rightarrow x_{0}$ e $d\left(z_{n}\right)=d_{n}$.

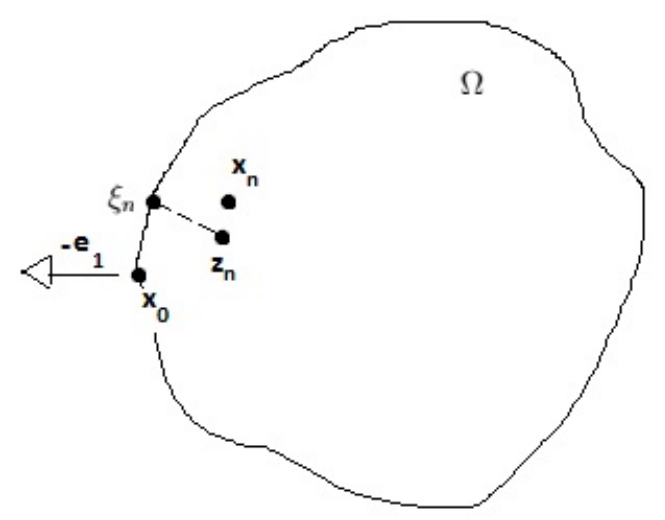

Figura 2.1:

Seja $\alpha_{n}=\alpha\left(z_{n}\right)$, definimos a função

$$
v_{n}(y)=d_{n}^{\alpha_{n}} u\left(\xi_{n}+d_{n} y\right)
$$

para $y \in \Omega_{n}:=\left\{y \in \mathbb{R}^{n} ; \xi_{n}+d_{n} y \in U\right\}$ onde $U$ é a vizinhança de $\partial \Omega$ dada pelo Lema (2.1.2). Observe que $\Omega_{n} \rightarrow D$ com $n \rightarrow \infty$, pois para $\xi_{n}+d_{n} y \in U$ como $x_{n} \rightarrow 0$ então 
$z_{n} \rightarrow 0 \operatorname{logo} d_{n}$ é bem pequeno, e se tivermos $y_{1}<0$ teremos que $\xi_{n}+d_{n} y$ não pertence a $U$. Então $y_{1}>0$. A função $v_{n}$ verifica a equação

$$
\triangle_{p} v=d_{n}^{\gamma\left(z_{n}\right)-\gamma\left(\xi_{n}+d_{n} y\right)} d_{n}^{\gamma\left(\xi_{n}+d_{n} y\right)} a\left(\xi_{n}+d_{n} y\right) v_{n}^{q} \quad \text { em } \Omega_{n}
$$

Pois

$$
\nabla v_{n}(y)=\left(d_{n}^{\alpha_{n}} \frac{\partial u}{\partial z_{1}}(z) d_{n}, \ldots, d_{n}^{\alpha_{n}} \frac{\partial u}{\partial z_{n}}(z) d_{n}\right)=d_{n}^{\alpha_{n}+1} \nabla u(z)
$$

onde $z=\xi_{n}+d_{n} y$

$$
\begin{aligned}
\left|\nabla v_{n}(y)\right|^{p-2} \nabla v_{n}(y) & =d_{n}^{\left(\alpha_{n}+1\right)(p-2)} d_{n}^{\alpha_{n}+1}|\nabla u(z)|^{p-2} \nabla u(z) \\
& =d_{n}^{\left(\alpha_{n}+1\right)(p-1)}|\nabla u(z)|^{p-2} \nabla u(z) \\
\operatorname{div}\left(\left|\nabla v_{n}(y)\right|^{p-2} \nabla v_{n}(y)\right) & =\sum_{i=1}^{n} \frac{\partial}{\partial y_{i}}\left(d_{n}^{\left(\alpha_{n}+1\right)(p-1)}|\nabla u(z)|^{p-2} \frac{\partial u}{\partial z_{i}}(z)\right) \\
& =d_{n}^{\left(\alpha_{n}+1\right)(p-1)} \sum_{i=1}^{n} \frac{\partial}{\partial z_{i}}\left(|\nabla u(z)|^{p-2} \frac{\partial u}{\partial z_{i}}(z)\right) d_{n} \\
& =d_{n}^{\left(\alpha_{n}+1\right)(p-1)+1} \sum_{i=1}^{n} \frac{\partial}{\partial z_{i}}\left(|\nabla u(z)|^{p-2} \frac{\partial u}{\partial z_{i}}(z)\right) \\
& =d_{n}^{\gamma\left(z_{n}\right)+\alpha\left(z_{n}\right) q} \triangle_{p} u(z) \\
& =d_{n}^{\gamma\left(z_{n}\right)+\alpha\left(z_{n}\right) q} a\left(\xi_{n}+d_{n} y\right) u\left(\xi_{n}+d_{n} y\right)^{q} \\
& =d_{n}^{\gamma\left(z_{n}\right)} a\left(\xi_{n}+d_{n} y\right)\left(d_{n}^{\alpha\left(z_{n}\right)} u\left(\xi_{n}+d_{n} y\right)\right)^{q} \\
& =d_{n}^{\gamma\left(z_{n}\right)} a\left(\xi_{n}+d_{n} y\right) v_{n}^{q}(y) \\
& =d_{n}^{\gamma\left(z_{n}\right)-\gamma\left(\xi_{n}+d_{n} y\right)} d_{n}^{\gamma\left(\xi_{n}+d_{n} y\right)} a\left(\xi_{n}+d_{n} y\right) v_{n}^{q}(y) \text { em } \Omega_{n} .
\end{aligned}
$$

Como $\gamma$ é Hölder contínua

$$
\begin{aligned}
\left|\gamma\left(z_{n}\right)-\gamma\left(\xi_{n}+d_{n} y\right)\right| & \leq C\left\|z_{n}-\left(\xi_{n}+d_{n} y\right)\right\|^{\mu}=C\left\|d_{n}\left(-\nu\left(\xi_{n}\right)\right)-d_{n} y\right\|^{\mu} \\
& =C\left(d_{n}\left\|\nu\left(\xi_{n}\right)+y\right\|\right)^{\mu} \leq C_{1} d_{n}^{\mu}
\end{aligned}
$$

para $y$ em um subconjunto limitado de $D$. O primeiro termo a direita de $(2.83)$ tende para 1 com $n \rightarrow \infty$, para $y$ em um subconjuto de $D$ e $d_{n}$ suficientemente pequeno. Pois, 
como vimos $\gamma\left(z_{n}\right)-\gamma\left(\xi_{n}+d_{n} y\right) \leq C_{1} d_{n}^{\mu}$ então

$$
d_{n}^{\gamma\left(z_{n}\right)-\gamma\left(\xi_{n}+d_{n} y\right)} \geq d_{n}^{C_{1} d_{n}^{\mu}}
$$

e $\lim _{n \rightarrow \infty} d_{n}^{C_{1} d_{n}^{\mu}}=1$ então $\lim _{n \rightarrow \infty} d_{n}^{\gamma\left(z_{n}\right)-\gamma\left(\xi_{n}+d_{n} y\right)} \geq 1$. Mas como

$$
\begin{aligned}
-C_{1} d_{n}^{\mu} & \leq \gamma\left(z_{n}\right)-\gamma\left(\xi_{n}+d_{n} y\right) \quad \Rightarrow \\
d_{n}^{-C_{1} d_{n}^{\mu}} \geq & \geq d_{n}^{\gamma\left(z_{n}\right)-\gamma\left(\xi_{n}+d_{n} y\right)}
\end{aligned}
$$

e como $\lim _{n \rightarrow \infty} d_{n}^{-C_{1} d_{n}^{\mu}}=1$ temos $\lim _{n \rightarrow \infty} d_{n}^{\gamma\left(z_{n}\right)-\gamma\left(\xi_{n}+d_{n} y\right)} \leq 1$. Assim, temos $\lim _{n \rightarrow \infty} d_{n}^{\gamma\left(z_{n}\right)-\gamma\left(\xi_{n}+d_{n} y\right)}=$ 1. E temos que $\frac{d\left(\xi_{n}+d_{n} y\right)}{d_{n}} \rightarrow y_{1}$ pois como $d\left(\varepsilon_{n}\right)=0$ e pelo Teorema do Valor Médio

$$
\begin{aligned}
d\left(\xi_{n}+d_{n} y\right)-d\left(\xi_{n}\right) & =\nabla d\left(\xi_{n}+\theta_{n} d_{n} y\right) d_{n} y \Rightarrow \\
\frac{d\left(\xi_{n}+d_{n} y\right)}{d_{n}} & =\nabla d\left(\xi_{n}+\theta_{n} d_{n} y\right) y \Rightarrow \\
\lim _{n \rightarrow \infty} \frac{d\left(\xi_{n}+d_{n} y\right)}{d_{n}} & =\nabla d(0) y
\end{aligned}
$$

no ponto 0 o gradiente de $d$ aponta no sentido que $d$ cresce, neste caso temos que $\nabla d(0)=$ $e_{1}$ assim

$$
\lim _{n \rightarrow \infty} \frac{d\left(\xi_{n}+d_{n} y\right)}{d_{n}}=e_{1} y=y_{1}
$$

e obtemos pela condição (2.2) e de (2.84) que

$$
\begin{aligned}
d_{n}^{\gamma\left(\xi_{n}+d_{n} y\right)} a\left(\xi_{n}+d_{n} y\right) & =a\left(\xi_{n}+d_{n} y\right) d\left(\xi_{n}+d_{n} y\right)^{\gamma\left(\xi_{n}+d_{n} y\right)}\left(\frac{d_{n}}{d\left(\xi_{n}+d_{n} y\right)}\right)^{\gamma\left(\xi_{n}+d_{n} y\right)}, \\
\lim _{n \rightarrow+\infty} d_{n}^{\gamma\left(\xi_{n}+d_{n} y\right)} a\left(\xi_{n}+d_{n} y\right) & =\lim _{n \rightarrow+\infty} a\left(\xi_{n}+d_{n} y\right) d\left(\xi_{n}+d_{n} y\right)^{\gamma\left(\xi_{n}+d_{n} y\right)}\left(\frac{d_{n}}{d\left(\xi_{n}+d_{n} y\right)}\right)^{\gamma\left(\xi_{n}+d_{n} y\right)}, \\
\lim _{n \rightarrow+\infty} d_{n}^{\gamma\left(\xi_{n}+d_{n} y\right)} a\left(\xi_{n}+d_{n} y\right) & =Q(0) y_{1}^{-\gamma(0)}=Q_{0} y_{1}^{-\gamma_{0}}
\end{aligned}
$$

para $y$ em um subconjunto limitado de $D$. Agora usando a estimativa provada no Lema(2.1.2), existe constantes positivas $C_{2}$ tal que

$$
v_{n}(y) \leq C_{2}\left(\frac{d_{n}}{d\left(\xi_{n}+d_{n} y\right)}\right)^{\alpha_{n}} d\left(\xi_{n}+d_{n} y\right)^{\alpha_{n}-\alpha\left(\xi_{n}+d_{n} y\right)}
$$


Pois do Lema (2.1.2), temos

$$
\begin{aligned}
u(x) & \leq C_{2} d(x)^{-\alpha(x)} \Rightarrow \\
u\left(\xi_{n}+d_{n} y\right) & \leq C_{2} d\left(\xi_{n}+d_{n} y\right)^{-\alpha\left(\xi_{n}+d_{n} y\right)} \Rightarrow \\
d_{n}^{\alpha_{n}} u\left(\xi_{n}+d_{n} y\right) & \leq d_{n}^{\alpha_{n}} C_{2} d\left(\xi_{n}+d_{n} y\right)^{-\alpha\left(\xi_{n}+d_{n} y\right)} \Rightarrow \\
v_{n}(y) & \leq C_{2} \frac{d_{n}^{\alpha_{n}}}{d\left(\xi_{n}+d_{n} y\right)^{\alpha_{n}}} d\left(\xi_{n}+d_{n} y\right)^{\alpha_{n}-\alpha\left(\xi_{n}+d_{n} y\right)} .
\end{aligned}
$$

E como

$$
\begin{aligned}
C_{1} d(x)^{-\alpha(x)} & \leq u(x) \Rightarrow \\
C_{1} d\left(\xi_{n}+d_{n} y\right)^{-\alpha\left(\xi_{n}+d_{n} y\right)} & \leq u\left(\xi_{n}+d_{n} y\right) \Rightarrow \\
C_{1} d_{n}^{\alpha_{n}} d\left(\xi_{n}+d_{n} y\right)^{-\alpha\left(\xi_{n}+d_{n} y\right)} & \leq d_{n}^{\alpha_{n}} u\left(\xi_{n}+d_{n} y\right) \Rightarrow \\
C_{1}\left(\frac{d_{n}}{d\left(\xi_{n}+d_{n} y\right)}\right)^{\alpha_{n}} d\left(\xi_{n}+d_{n} y\right)^{\alpha_{n}-\alpha\left(\xi_{n}+d_{n} y\right)} & \leq v_{n}(y) .
\end{aligned}
$$

Como o lado direito de (2.85) converge para $C_{2} y_{1}^{-\alpha_{0}}$ pois $\alpha(x)=\frac{p-\gamma(x)}{q-p+1}$ então

$$
\begin{aligned}
\alpha_{n}-\alpha\left(\xi_{n}+d_{n} y\right) & =\frac{p-\gamma\left(z_{n}\right)-p+\gamma\left(\xi_{n}+d_{n} y\right)}{q-p+1}=\frac{\gamma\left(\xi_{n}+d_{n} y\right)-\gamma\left(z_{n}\right)}{q-p+1} \\
\lim _{n \rightarrow \infty} d\left(\xi_{n}+d_{n} y\right)^{\alpha_{n}-\alpha\left(\xi_{n}+d_{n} y\right)} & =\lim _{n \rightarrow \infty}\left[d\left(\xi_{n}+d_{n} y\right)^{\gamma\left(z_{n}\right)-\gamma\left(\xi_{n}+d_{n} y\right)}\right]^{\frac{-1}{q-p+1}}=1
\end{aligned}
$$

e o lado esquerdo de (2.86) converge para $C_{1} y_{1}^{-\alpha_{0}}$. Obtemos que a sequência $v_{n}$ é uniformemente limitada em um subconjunto compacto de $D$. Passando para uma subsequência de $v_{n}$ que converge em $C^{1}(D)$ para uma função $v$ que verifica

$$
\left\{\begin{array}{l}
\triangle_{p} v=Q_{0} y_{1}^{-\gamma_{0}} v^{q}, \quad x \in D \\
v=+\infty, \quad x \in \partial D
\end{array}\right.
$$

e $v=+\infty$ na $\partial D$ pois de $(2.86)$

$$
\begin{aligned}
C_{1}\left(\frac{d_{n}}{d\left(\xi_{n}+d_{n} y\right)}\right)^{\alpha_{n}} d\left(\xi_{n}+d_{n} y\right)^{\alpha_{n}-\alpha\left(\xi_{n}+d_{n} y\right)} & \leq v_{n}(y) \\
C_{1} y_{1}^{-\alpha_{0}} & \leq \lim _{n \rightarrow \infty} v_{n}(y)=v(y)
\end{aligned}
$$

onde $\alpha_{0}=\alpha(0)>0$, mas como $y \in \partial D$ temos $y_{1}=0$ logo $\infty \leq v(y)$. E de (2.85) e (2.86) concluímos

$$
C_{1} y_{1}^{-\alpha_{0}} \leq v \leq C_{2} y_{1}^{-\alpha_{0}}
$$


e do Teorema (2.1.2), temos

$$
v(y)=\left(\frac{(p-1) \alpha_{0}^{p-1}\left(\alpha_{0}+1\right)}{Q_{0}}\right)^{\frac{1}{q-p+1}} y_{1}^{-\alpha_{0}}
$$

tomando $y=e_{1}$, temos

$$
v\left(e_{1}\right)=\left(\frac{(p-1) \alpha_{0}^{p-1}\left(\alpha_{0}+1\right)}{Q_{0}}\right)^{\frac{1}{q-p+1}} .
$$

Além disso,

$$
v_{n}\left(e_{1}\right)=d_{n}^{\alpha_{n}} u\left(\xi_{n}+d_{n} e_{1}\right)=d_{n}^{\alpha_{n}} u\left(x_{n}\right)
$$

tirando o limite dos dois lados de (2.88) temos

$$
\lim _{x_{n} \rightarrow x_{0}} d_{n}^{\alpha_{n}} u\left(x_{n}\right)=v\left(e_{1}\right)
$$

Usando o Teorema do Valor Médio existe $\theta_{n} \in t\left(x_{n}\right)+(1-t) \xi_{n}$ com $t \in[0,1]$ tal que

$$
\begin{aligned}
\left(\frac{d\left(x_{n}\right)}{d\left(z_{n}\right)}\right)^{\alpha\left(x_{n}\right)} & =\left(\frac{d\left(x_{n}\right)-d\left(\xi_{n}\right)}{d\left(z_{n}\right)}\right)^{\alpha\left(x_{n}\right)}=\left(\frac{\left|<\nabla d_{n}\left(\theta_{n}\right), d_{n} e_{1}>\right|}{d\left(z_{n}\right)}\right)^{\alpha\left(x_{n}\right)} \\
& =\left(\left|<\nabla d_{n}\left(\theta_{n}\right), e_{1}>\right|\right)^{\alpha\left(x_{n}\right)}
\end{aligned}
$$

tomando $n \rightarrow \infty$ temos

$$
\lim _{x_{n} \rightarrow x_{0}} d\left(x_{n}\right)^{\alpha\left(z_{n}\right)} d\left(z_{n}\right)^{-\alpha\left(z_{n}\right)}=\left|<e_{1}, e_{1}>\right|^{\alpha(0)}=1 .
$$

Pelo fato de $\lim _{x_{n} \rightarrow x_{0}} d\left(x_{n}\right)^{\alpha\left(x_{n}\right)-\alpha\left(z_{n}\right)}=1$, de (2.89) e de (2.90) temos que

$\lim _{x_{n} \rightarrow x_{0}} d\left(x_{n}\right)^{\alpha\left(x_{n}\right)} u\left(x_{n}\right)=\lim _{x_{n} \rightarrow x_{0}} d\left(x_{n}\right)^{\alpha\left(x_{n}\right)-\alpha\left(z_{n}\right)}\left(d\left(x_{n}\right)^{\alpha\left(z_{n}\right)} d\left(z_{n}\right)^{-\alpha\left(z_{n}\right)}\right) d\left(z_{n}\right)^{\alpha\left(z_{n}\right)} u\left(x_{n}\right)=v\left(e_{1}\right)$

assim, provamos a estimativa (2.3).

\subsection{Unicidade do Teorema (2.1)}

Sejam $u$ e $v$ soluções positivas de (2.1). De acordo com a estimativa (2.3) temos

$$
\lim _{x \rightarrow x_{0}} d(x)^{\alpha(x)} u(x) d(x)^{-\alpha(x)} v(x)^{-1}=v\left(e_{1}\right) v\left(e_{1}\right)^{-1}=1 \quad \Rightarrow \quad \lim _{x \rightarrow x_{0}} \frac{u(x)}{v(x)}=1
$$


para todo $x_{0} \in \partial \Omega$. Dado $\varepsilon>0 \exists \delta>0$ tal que $\left\|x-x_{0}\right\| \leq \delta$ temos

$$
\begin{aligned}
\left|\frac{u(x)}{v(x)}-1\right| & <\varepsilon \Rightarrow-\varepsilon<\frac{u(x)}{v(x)}-1<\varepsilon \Rightarrow \\
1-\varepsilon & <\frac{u(x)}{v(x)}<1+\varepsilon \Rightarrow \\
(1-\varepsilon) v(x) & <u(x)<(1+\varepsilon) v(x)
\end{aligned}
$$

se $\left\|x-x_{0}\right\| \leq \delta$, ou seja, $d(x) \leq \delta$.

Denote $\Omega^{\delta}:=\{x \in \Omega ; d(x)>\delta\}$ e considere

$$
\left\{\begin{array}{l}
\triangle_{p} w=a(x) w^{q}, \quad x \in \Omega^{\delta}, \\
w=u, \quad x \in \partial \Omega^{\delta}
\end{array}\right.
$$

esse problema tem solução, pois $\underline{w}=0$ é subsolução e $\bar{w}=\max _{\partial \Omega^{\delta}} u(x)$ é supersolução, e a solução é única já que podemos usar comparação duas vezes para mostrar que são iguais. Essa solução é claramente $w=u$. Por outro lado como $q>p-1$, as funções $(1-\varepsilon) v$ e $(1+\varepsilon) v$ são sub e supersolução de (2.92). Pois

$$
\begin{aligned}
\underline{u} & =(1-\varepsilon) v \\
\triangle_{p} \underline{u} & =(1-\varepsilon)^{p-1} \triangle_{p} v
\end{aligned}
$$

como $p-1<q$ e $1-\varepsilon<1 \Rightarrow(1-\varepsilon)^{p-1}>(1-\varepsilon)^{q}$, obtemos

$$
\triangle_{p} \underline{u}=(1-\varepsilon)^{p-1} a(x) v^{q} \geq(1-\varepsilon)^{q} a(x) v^{q}=a(x) \underline{u}^{q}
$$

na $\partial \Omega^{\delta}$

$$
\underline{u}=(1-\varepsilon) v \leq u
$$

então $\underline{u}$ é subsolução.

Onde $\bar{u}=(1+\varepsilon) v$ é supersolução pois

$$
\triangle_{p} \bar{u}=(1+\varepsilon)^{p-1} \triangle_{p} v
$$

como $p-1<q$ e $(1+\varepsilon)>1 \Rightarrow(1+\varepsilon)^{p-1}<(1+\varepsilon)^{q}$, assim

$$
\triangle_{p} \bar{u}=(1+\varepsilon)^{p-1} \triangle_{p} v \leq(1+\varepsilon)^{q} a(x) v^{q}=a(x) \bar{u}^{q}
$$

na $\partial \Omega^{\delta}$

$$
\bar{u}=(1+\varepsilon) v \geq u
$$


então $\bar{u}$ é supersolução. Assim (2.91) vale em $\Omega^{\delta}, \operatorname{logo}$

$$
(1-\varepsilon) v(x) \leq u(x) \leq(1+\varepsilon) v(x) .
$$

Portanto de (2.91) e (2.93) temos

$$
(1-\varepsilon) v(x) \leq u(x) \leq(1+\varepsilon) v(x), \quad \text { em } \Omega
$$

tomando $\varepsilon \rightarrow 0$ temos $v=u$ em $\Omega$. 


\section{Capítulo}

\section{Soluções Inteiras para Problemas} Elípticos não Lineares com Expoentes Variáveis

Vamos estudar nesse capítulo a existência de solução positiva do problema

$$
\left\{\begin{array}{l}
\triangle u=u^{q(x)} \quad x \in \mathbb{R}^{N} \\
u(x) \rightarrow \infty, \quad \operatorname{com}|x| \rightarrow \infty
\end{array}\right.
$$

que é chamada grande solução inteira, pois $\lim _{|x| \rightarrow \infty} u(x)=+\infty$. Onde $N \geq 3$, e $q: \mathbb{R}^{N} \rightarrow$ $[0, \infty)$ é localmente Hölder contínua, com $q(x) \leq 1$ para $|x| \rightarrow \infty$.

Para mostrar que existe solução do problema (3.1), vamos precisar de alguns resultados preliminares.

\subsection{Resultados Preliminares}

Nessa seção vamos mostrar a existência de solução para o problema

$$
\left\{\begin{array}{l}
u^{\prime \prime}(r)+\frac{N-1}{r} u^{\prime}(r)=g(u(r), r), \\
u(0)=a>0 \\
u^{\prime}(0)=0
\end{array}\right.
$$


onde $g$ satisfaz algumas condições. Para isso, vamos mostrar que existe uma solução positiva que satisfaz

$$
u(r)=a+\int_{0}^{r} t^{1-N} \int_{0}^{t} s^{N-1} g(u(s), s) d s
$$

onde $g:(0, \infty) \times[0, \infty) \rightarrow(0, \infty)$ é uma função contínua que satisfaz uma ou mais das seguintes condições :

G1 - A função $g(t, r)$ é não decrescente em $t$, para um $r$ fixado. Mais ainda, para cada $t>0$, fixado existe uma constante $c_{t}$ que depende de $t$, tal que $g(t, r)>c_{t}$ para todo $r \geq 0$.

G2 - Existe uma função não decrescente, diferenciável $f:(0, \infty) \rightarrow(0, \infty)$ satisfazendo

$$
\int_{0}^{1} \frac{1}{f(t)} d t=+\infty \quad e \quad \int_{1}^{\infty} \frac{1}{f(t)} d t<\infty
$$

e existem constantes $R \geq 0$ e $0<\beta \leq 1$ tais que

$$
g(t, r) \leq \begin{cases}f(t) & \text { para } 0 \leq r \leq R, \quad t \geq 0 \\ t^{\beta} & \text { para } r \geq R, \quad t \geq 1\end{cases}
$$

G3 - Existe uma função localmente limitada $\mu:(0, \infty) \times(0, \infty) \rightarrow(0, \infty)$ tal que

$$
|g(t, r)-g(s, r)| \leq \mu(t, s)|t-s|, \quad \forall t, s \in(0, \infty), 0 \leq r \leq R,
$$

onde $R$ foi definido em G2.

Observação Quando $R=0$ em G2, a condição (3.4) e a condição G3 são vazias.

Lema 3.1.1 Suponha que g satisfaz $\mathbf{G 1 , G 2}$ com $R=0$ em G2. Então, para qualquer $a>0$, a equação (3.3) tem uma solução positiva u definida para todo $r \geq 0$. Além disso, $u(r) \rightarrow \infty \operatorname{com} r \rightarrow \infty$.

Prova Pelo Apêndice B conseguimos garantir a existência de uma função $u(r)$, para $r$ pequeno, que satisfaz (3.3). Seja $\left[0, R_{a}\right)$ o intervalo maximal de existência de $u(r)$. Vamos mostrar que $R_{a}=+\infty$. Como $g$ é não decrescente em relação a primeira variável, para $s$ fixado, e $u$ é não decrescente, pois $u^{\prime}=r^{1-N} \int_{0}^{r} s^{N-1} g(u(s), s) d s$ e como $r \geq 0$ e $g>0$, 
temos que $u^{\prime} \geq 0$, e para qualquer $r<R_{a}$

$$
\begin{aligned}
u(r) & =a+\int_{0}^{r} t^{1-N} \int_{0}^{t} s^{N-1} g(u(s), s) d s d t \\
& \leq a+\int_{0}^{r} t^{1-N} \int_{0}^{t} s^{N-1} g(u(s)+1, s) d s d t \\
& \leq a+\int_{0}^{r} t^{1-N} \int_{0}^{t} s^{N-1}(u(s)+1)^{\beta} d s d t, \quad \text { por G2 } \\
& \leq a+\int_{0}^{r} t^{1-N} \int_{0}^{t} t^{N-1}(u(t)+1)^{\beta} d s d t, \quad \text { já que } u \text { não decrescente e } s<t \\
& =a+\int_{0}^{r} t^{1-N} t^{N-1}(u(t)+1)^{\beta} t d t \\
& =a+\int_{0}^{r} t(u(t)+1)^{\beta} d t \\
& \leq a+\int_{0}^{r} t(u(t)+1) d t, \quad \text { pois } 0<\beta \leq 1 \\
& =a+\int_{0}^{r} t d t+\int_{0}^{r} t u(t) d t .
\end{aligned}
$$

Pela desigualdade de Gronwall generalizada temos

$$
\begin{aligned}
u(r) & \leq\left(a+\int_{0}^{r} t d t\right) e^{\int_{0}^{r} s d s} \\
& =\left(a+\left.\frac{t^{2}}{2}\right|_{0} ^{r}\right) e^{\frac{r^{2}}{2}} \\
& =\left(a+\frac{r^{2}}{2}\right) e^{\frac{r^{2}}{2}}
\end{aligned}
$$

para $r \in\left[0, R_{a}\right)$ então $u(r) \leq C$ e

$$
u^{\prime}(r)=r^{1-N} \int_{0}^{r} s^{N-1} g(u(s), s) d s \leq r^{1-N} r^{N-1}(u(r)+1)^{\beta} \int_{0}^{r} d s=r(u(r)+1)^{\beta} \leq C_{1}
$$

pois $u$ é limitado e $r \in\left[0, R_{a}\right)$. Então $u$ e $u^{\prime}$ são limitados, logo podemos estender a função $u$ em um intervalo maior que $\left[0, R_{a}\right)$ o que contradiz o fato desse intervalo ser o maximal. Então $R_{a}=\infty$. De $\mathbf{G} \mathbf{1}$, temos que para um $t$ fixo existe $c_{t}$ tal que $g(t, r) \geq c_{t}$, 
assim

$$
\begin{aligned}
u(r) & =a+\int_{0}^{r} t^{1-N} \int_{0}^{t} s^{N-1} g(u(s), s) d s d t \\
& \geq a+\int_{0}^{r} t^{1-N} \int_{0}^{t} s^{N-1} g(a, s) d s d t, \quad \text { pois } u(r) \geq a \\
& \geq a+\int_{0}^{r} t^{1-N} \int_{0}^{t} s^{N-1} c_{a} d s d t \\
& =a+\frac{1}{N} \int_{0}^{r} t c_{a} d t \\
& =a+\left.\frac{c_{a} t^{2}}{2 N}\right|_{0} ^{r} \\
& =a+C_{2} r^{2}
\end{aligned}
$$

então quando $r \rightarrow \infty u(r) \rightarrow \infty$.

Lema 3.1.2 Suponha g satisfaz as condições $\boldsymbol{G 1}$ e G2 para qualquer $R>0$. Então existe $a>0$ tal que a equação (3.3) tem uma solução positiva u definida para todo $r \geq 0$. Além disso, $u(r) \rightarrow+\infty$ com $r \rightarrow+\infty$.

Prova: Se uma solução de (3.3) positiva $u$ existe para todo $r$, então $\lim _{r \rightarrow+\infty} u(r)=+\infty$, pois $u(r)$ é uma função não decrescente. Vamos mostrar que existe uma solução de (3.3) para todo $r \geq 0$. Observe que para cada $a>0$, a equação (3.3) tem uma solução $u_{a}$ em um intervalo $\left[0, R_{a}\right)$. Suponha que $\left[0, R_{a}\right)$ é um intervalo maximal de existência para a solução $u_{a}$. Vamos mostrar que $R_{a}>R$, onde $R$ foi definido em G2, e $a>0$. Suponha que $R_{a} \leq R$ para todo $a>0$. Como $\left[0, R_{a}\right)$ é um intervalo maximal de existência de $u_{a}$, e $u_{a}$ é positiva e não decrescente temos que $\lim _{r \rightarrow R_{a}^{-}} u_{a}(r)=+\infty$, pois se fosse finito pode ser estendido para $R_{a}$. Para $0 \leq s \leq R_{a} \leq R$, temos $g\left(u_{a}(s), s\right) \leq f\left(u_{a}(s)\right)$ de G2. Diferenciando (3.3), usando a condição G2, o fato de $u$ e $f$ são não decrescente temos,

$$
\begin{aligned}
u_{a}^{\prime}(r) & =r^{1-N} \int_{0}^{r} s^{N-1} g\left(u_{a}(s), s\right) d s \\
& \leq r^{1-N} \int_{0}^{r} s^{N-1} f\left(u_{a}(s)\right) d s
\end{aligned}
$$




$$
\begin{aligned}
\stackrel{s<r}{<} r^{1-N} \int_{0}^{r} r^{N-1} f\left(u_{a}(r)\right) d s \\
=f\left(u_{a}(r)\right) r \quad \Rightarrow \\
\frac{1}{f\left(u_{a}(r)\right)} \leq \frac{r}{u_{a}^{\prime}(r)}
\end{aligned}
$$

em $\left[0, R_{a}\right)$ tal que

$$
\frac{u_{a}^{\prime}(r)}{f\left(u_{a}(r)\right)} \leq r
$$

integrando sobre o intervalo $[0, \eta)$ para $\eta<R_{a}$ temos

$$
\int_{0}^{\eta} \frac{u_{a}^{\prime}(r)}{f\left(u_{a}(r)\right)} d r \leq \frac{\eta^{2}}{2}
$$

como

$$
\frac{u_{a}^{\prime}(r)}{f\left(u_{a}(r)\right)}=\frac{d}{d r} \int_{a}^{u_{a}(r)} \frac{1}{f(t)} d t
$$

temos

$$
\begin{gathered}
\int_{0}^{\eta} \frac{u_{a}^{\prime}(r)}{f\left(u_{a}(r)\right)} d r=\int_{a}^{u_{a}(\eta)} \frac{1}{f(t)} d t \leq \frac{\eta^{2}}{2} \leq \frac{R_{a}^{2}}{2} \leq \frac{R^{2}}{2} \Rightarrow \\
\lim _{\eta \rightarrow R_{a}^{-}} \int_{a}^{u_{a}(\eta)} \frac{1}{f(t)} d t \leq \lim _{\eta \rightarrow R_{a}^{-}} \frac{\eta^{2}}{2} \leq \frac{R^{2}}{2} \Rightarrow \\
\int_{a}^{\infty} \frac{1}{f(t)} d t \leq \frac{R^{2}}{2}<\infty .
\end{gathered}
$$

Com isso, para todo $a>0$, obtemos

$$
\lim _{a \rightarrow 0} \int_{a}^{\infty} \frac{1}{f(t)} d t<\infty
$$

de (3.4) temos que o limite a esquerda da desigualdade acima é infinito, obtemos então, uma contradição. Portanto (3.3) tem uma solução válida em $[0, R]$ para algum $a>0$.

Vamos mostrar que essa solução é, de fato, válida para todo $r \geq 0$. Como o $a$ já foi escolhido, vamos suprimir a dependência do $a$.

Como $u$ existe e é contínua em $[0, R]$, nós temos $u(R)<\infty$ tal que $u^{\prime}(R)<\infty\left(u^{\prime}(R) \leq\right.$ $\left.\frac{R}{N} f\left(u_{a}(R)\right)<\infty\right)$, então a solução $u$ de $(3.3)$ pode ser estendida para o intervalo $[0, R+$ $\varepsilon) \operatorname{com} \varepsilon>0$. 
Seja $R_{0}=\sup \{r ; u$ é uma solução de $(3.3)$ em $[0, r)\}$. Claramente $R_{0}>R$. Se $R_{0}=$ $\infty$ acabamos. Suponha $R_{0}<\infty$ tal que $\lim _{r \rightarrow R_{0}^{-}} u(r)=+\infty$. Para $r<R_{0}$, temos

$$
\begin{aligned}
& u(r)=a+\int_{0}^{r} t^{1-N} \int_{0}^{t} s^{N-1} g(u(s), s) d s d t \\
& =a+\int_{0}^{R} t^{1-N} \int_{0}^{t} s^{N-1} g(u(s), s) d s d t+\int_{R}^{r} t^{1-N} \int_{0}^{t} s^{N-1} g(u(s), s) d s d t \\
& =a+\int_{0}^{R} t^{1-N} \int_{0}^{t} s^{N-1} g(u(s), s) d s d t+\int_{R}^{r} t^{1-N} \int_{0}^{R} s^{N-1} g(u(s), s) d s d t \\
& +\int_{R}^{r} t^{1-N} \int_{R}^{t} s^{N-1} g(u(s), s) d s d t \\
& =u(R)+\int_{R}^{r} t^{1-N} \int_{0}^{R} s^{N-1} g(u(s), s) d s d t+\int_{R}^{r} t^{1-N} \int_{R}^{t} s^{N-1} g(u(s), s) d s d t \\
& =u(R)+\left(\int_{0}^{R} s^{N-1} g(u(s), s) d s\right)\left(\left.\frac{t^{2-N}}{2-N}\right|_{R} ^{r}\right)+\int_{R}^{r} t^{1-N} \int_{R}^{t} s^{N-1} g(u(s), s) d s d t \\
& =u(R)+\frac{r^{2-N}-R^{2-N}}{2-N} \int_{0}^{R} s^{N-1} g(u(s), s) d s+\int_{R}^{r} t^{1-N} \int_{R}^{t} s^{N-1} g(u(s), s) d s d t \\
& =u(R)+\frac{1}{N-2}\left(1-\left(\frac{r}{R}\right)^{2-N}\right) R^{2-N} \int_{0}^{R} s^{N-1} g(u(s), s) d s \\
& +\int_{R}^{r} t^{1-N} \int_{R}^{t} s^{N-1} g(u(s), s) d s d t \\
& =u(R)+\frac{1}{N-2}\left(1-\left(\frac{r}{R}\right)^{2-N}\right) R u^{\prime}(R)+\int_{R}^{r} t^{1-N} \int_{R}^{t} s^{N-1} g(u(s), s) d s d t .
\end{aligned}
$$

Usando a inequação provada no Apêndice (C)

$$
\int_{R}^{r} t^{1-N} \int_{R}^{t} s^{N-1} g(u(s), s) d s d t \leq \int_{R}^{r} s g(u(s), s) d s
$$

e as condições G1 que garantem $g$ é não decrescente, ou seja, $g(u(s), s) \leq g(u(s)+1, s)$

e G2 que para $R \leq s$ garante $g(u(s)+1, s) \leq(u(s)+1)^{\beta}$, obtemos 


$$
\begin{aligned}
u(r) & \leq u(R)+\frac{R u^{\prime}(R)}{N-2}\left(1-\left(\frac{R}{r}\right)^{N-2}\right)+\int_{R}^{r} s(u(s)+1)^{\beta} d s \\
& \leq u(R)+\frac{R u^{\prime}(R)}{N-2}+\int_{R}^{r} s(u(s)+1)^{\beta} d s \\
& \leq u^{*}(R)+\int_{R}^{r} s(u(s)+1) d s \\
& \leq u^{*}(R)+\int_{R}^{r} s d s+\int_{R}^{r} s u(s) d s .
\end{aligned}
$$

Pela desigualdade de Gronwall generalizada

$$
\begin{aligned}
u(r) & \leq\left(u^{*}(R)+\int_{R}^{r} s d s\right) e^{\int_{R}^{r} s d s} \\
& =\left(u^{*}(R)+\left.\frac{s^{2}}{2}\right|_{R} ^{r}\right) e^{\frac{r^{2}-R^{2}}{2}} \\
& =\left(u^{*}(R)+\frac{r^{2}-R^{2}}{2}\right) e^{\frac{r^{2}-R^{2}}{2}}
\end{aligned}
$$

para $r \in\left[0, R_{0}\right)$, então $u(r) \leq c$, onde $u^{*}(R)=u(R)+\frac{R u^{\prime}(R)}{N-2}$.

Provamos que $u$ é uniformemente limitado em $\left[0, R_{0}\right)$. Mas tinhamos que $\lim _{r \rightarrow R_{0}^{-}} u(r)=$ $+\infty$, contradição. Então $R_{0}=+\infty$.

Lema 3.1.3 Suponha que g satisfaz as condições $\mathbf{G 1}$, G2 e G3. Então, dado alguma constante $M>0$, existe $a>0$ tal que a solução inteira positiva $u_{a}$ de (3.3) correspondente ao valor a, satisfaz $u_{a}(R)>M$.

Prova: Como visto no Apêndice (B), garantimos a existência de uma solução $u$ para a equação (3.3) em um intervalo $\left[0, R_{a}\right)$.

Seja $A=\sup \{a>0 ; \quad$ equação (3.3) tem uma solução inteira positiva $\}$. Se $A=+\infty$, pela definição de solução inteira, temos que para todo $a>0, u_{a}(r)$ é solução de (3.3) para todo $r \in[0, \infty)$. Tome $a>M$, assim 


$$
\begin{aligned}
u_{a}(R) & =a+\int_{0}^{R} t^{1-N} \int_{0}^{t} s^{N-1} g(u(s), s) d s d t \\
& >M+\int_{0}^{R} t^{1-N} \int_{0}^{t} s^{N-1} g(u(s), s) d s d t \\
& \geq M
\end{aligned}
$$

então $u_{a}(R)>M$.

Por outro lado suponha $A<\infty$. Primeiramente, provaremos que existe $R_{A} \leq R$ tal que $\lim _{r \rightarrow R^{-}} u_{A}(r)=+\infty$, onde $R$ foi definido em G2. Observe que para qualquer $a>A$, a solução $u_{a}$ é blow up para algum $R_{a}>0$. E como $u_{a}$ é crescente em $a$, isto é, se $a<a^{\prime}$ então $u_{a}<u_{a^{\prime}}$, temos $R_{a^{\prime}} \leq R_{a}$ (pois se $R_{a}<R_{a^{\prime}} \Rightarrow \infty=u_{a}\left(R_{a}\right)<u_{a^{\prime}}\left(R_{a}\right)<\infty$ absurdo). E pela definição de intervalo maximal de solução, temos que $R_{a}=\inf \left\{r>0 ; u_{a}(r)=+\infty\right\}$. Em adição $R_{a} \leq R$ (pois $a>A$ e se $R_{a}>R$ então pela prova do Lema (3.1.2), $u_{a}(r)$ existe para todo $r$, ou seja $u_{a}$ é solução inteira, mas como $a>A$ temos que $u_{a}$ não é solução inteira). Como $R_{a^{\prime}} \leq R_{a}$ para $a<a^{\prime}$ e $R_{a} \leq R \forall a>A$ temos que $\lim _{a \rightarrow A^{+}} R_{a}$ existe e $\lim _{a \rightarrow A^{+}} R_{a}=R_{A} \leq R$.

AfirmaÇão $1 \lim _{a \rightarrow A} u_{a}(r)=u_{A}(r), \quad \forall r \in\left[0, R_{A}\right)$ e que esta convergência é uniforme num subconjunto compacto de $\left[0, R_{A}\right)$.

Pelo Apêndice (D) e pela condição G3

$$
\begin{aligned}
\left|u_{a}(r)-u_{A}(r)\right| & \leq|a-A|+\int_{0}^{r} t^{1-N} \int_{0}^{t} s^{N-1}\left|g\left(u_{a}(s), s\right)-g\left(u_{A}(s), s\right)\right| d s d t \\
& \leq|a-A|+\int_{0}^{r} s\left|g\left(u_{a}(s), s\right)-g\left(u_{A}(s), s\right)\right| d s \\
& \leq|a-A|+\int_{0}^{r} s \mu\left(u_{a}(s), u_{A}(s)\right)\left|u_{a}(s)-u_{A}(s)\right| d s .
\end{aligned}
$$

Seja $\delta>0$ dado, vamos mostrar que $\lim _{a \rightarrow A} u_{a}=u_{A}$ uniformemente num intervalo fechado $\left[0, R_{A}-\delta\right]$. Então para $s \in\left[0, R_{A}-\delta\right]$ temos que

$$
A \leq u_{A}(s) \leq u_{a}(s) \leq u_{a}\left(R_{A}-\delta\right)
$$

Como $\mu$ é localmente limitada existe uma constante $M_{\delta}>0$ tal que

$$
\mu\left(u_{a}(s), u_{A}(s)\right) \leq M_{\delta} \quad 0 \leq s \leq R_{A}-\delta
$$


Portanto de (3.6)

$$
\left|u_{a}(r)-u_{A}(r)\right| \leq|a-A|+M_{\delta} \int_{0}^{r} s\left|u_{a}(s)-u_{A}(s)\right| d s .
$$

Pela desigualdade de Gronwall temos

$$
\begin{aligned}
\left|u_{a}(r)-u_{A}(r)\right| & \leq|a-A|+M_{\delta} \int_{0}^{r} s|a-A| e^{\int_{s}^{r} M_{\delta} u d u} d s \\
& =|a-A|+M_{\delta}|a-A| \int_{0}^{r} e^{\left.\frac{M_{\delta} u^{2}}{2}\right|_{s} ^{r} s d s} \\
& =|a-A|+M_{\delta}|a-A| \int_{0}^{r} e^{\frac{M_{\delta}\left(r^{2}-s^{2}\right)}{2}} s d s \\
& =|a-A|+\left.M_{\delta}|a-A|\left(\frac{-e^{\frac{M_{\delta}\left(r^{2}-s^{2}\right)}{2}}}{M_{\delta}}\right)\right|_{0} ^{r} \\
& =|a-A|+M_{\delta}|a-A| \frac{\left(-1+e^{\frac{M_{\delta} r^{2}}{2}}\right)}{M_{\delta}} \\
& =|a-A| e^{\frac{M_{\delta} r^{2}}{2}}
\end{aligned}
$$

para $r \in\left[0, R_{A}-\delta\right]$. De (3.8) concluímos $\lim _{a \rightarrow A} u_{a}(r)=u_{A}(r)$ para todo $r \in\left[0, R_{A}\right)$ com a convergência sendo uniforme num subconjunto compacto de $\left[0, R_{A}\right)$.

AfIRMAÇ̃̃o $2 \lim _{r \rightarrow R_{A}^{-}} u_{A}(r)=+\infty$

Observe que de $\mathbf{G} \mathbf{2}$, temos $g\left(u_{a}(r), r\right) \leq f\left(u_{a}(r)\right)$ para todo $0 \leq r \leq R_{A} \leq R$ e defina a função $F:(0, \infty) \rightarrow(0, \infty)$ por

$$
F(z)=\int_{z}^{\infty} \frac{d s}{f(s)}
$$

Observe que $F^{\prime}<0$ e $F^{\prime \prime} \geq 0$

$$
\begin{aligned}
F^{\prime}(z) & =-\frac{1}{f(z)}<0 \\
F^{\prime \prime}(z) & =\frac{f^{\prime}(z)}{f(z)^{2}} \geq 0 \text { pois } f \text { é não decrescente }
\end{aligned}
$$

e para qualquer $a>A$ e $r<R_{a}$, temos que 


$$
\begin{gathered}
F^{\prime \prime}\left(u_{a}(r)\right)=\frac{\partial}{\partial r}\left(\frac{\partial F\left(u_{a}(r)\right)}{\partial u_{a}} u_{a}^{\prime}\right)=\frac{\partial}{\partial r}\left(\frac{\partial F\left(u_{a}(r)\right)}{\partial u_{a}}\right) u_{a}^{\prime}+\frac{\partial F\left(u_{a}(r)\right)}{\partial u_{a}} u_{a}^{\prime \prime}=\frac{\partial^{2} F\left(u_{a}(r)\right)}{\partial u_{a}^{2}} u_{a}^{\prime 2} \\
+\frac{\partial F\left(u_{a}(r)\right)}{\partial u_{a}} u_{a}^{\prime \prime} \geq \frac{\partial F\left(u_{a}(r)\right)}{\partial u_{a}} u_{a}^{\prime \prime}=-\frac{u_{a}^{\prime \prime}(r)}{f\left(u_{a}(r)\right)} .
\end{gathered}
$$

Como

$$
\begin{aligned}
& u_{a}^{\prime}(r)=r^{1-N} \int_{0}^{r} s^{N-1} g\left(u_{a}(s), s\right) d s \\
& u_{a}^{\prime \prime}(r)=(1-N) r^{-N} \int_{0}^{r} s^{N-1} g\left(u_{a}(s), s\right) d s+r^{1-N}\left(r^{N-1} g\left(u_{a}(r), r\right)\right) \leq g\left(u_{a}(r), r\right) .
\end{aligned}
$$

Assim

$$
\frac{\partial F\left(u_{a}(r)\right)}{\partial u_{a}} u_{a}^{\prime \prime} \geq \frac{-g\left(u_{a}(r), r\right)}{f\left(u_{a}(r)\right)} \geq-1 \Rightarrow \int_{0}^{r} \frac{\partial F\left(u_{a}(r)\right)}{\partial u_{a}} u_{a}^{\prime \prime} d s \geq \int_{0}^{r}-d s .
$$

Integrando por partes o lado esquerdo de (3.9), temos

$$
\begin{array}{rlrl}
u(s)=\frac{\partial F}{\partial u_{a}}\left(u_{a}(s)\right) & d u & =\frac{\partial^{2} F}{\partial u_{a}^{2}}\left(u_{a}(s)\right) u_{a}^{\prime}(s) d s \\
d v & =u_{a}^{\prime \prime}(s) d s & v & =u_{a}^{\prime}(s)
\end{array}
$$

assim

$$
\begin{array}{r}
\left.u_{a}^{\prime}(s) \frac{\partial F}{\partial u_{a}}\left(u_{a}(s)\right)\right|_{0} ^{r}-\int_{0}^{r} u_{a}^{\prime}(s) \frac{\partial^{2} F}{\partial u_{a}^{2}} u_{a}^{\prime}(s) d s \geq-r \quad \Rightarrow \\
u_{a}^{\prime}(r) \frac{\partial F}{\partial u_{a}}\left(u_{a}(r)\right) \geq u_{a}^{\prime}(r) \frac{\partial F}{\partial u_{a}}\left(u_{a}(r)\right)-\int_{0}^{r} u_{a}^{\prime 2}(s) \frac{\partial^{2} F}{\partial u_{a}^{2}} d s \geq-r \quad \Rightarrow \\
u_{a}^{\prime}(r) \frac{\partial F}{\partial u_{a}}\left(u_{a}(r)\right) \geq-r \quad \Rightarrow \quad \frac{\partial F}{\partial r}\left(u_{a}(r)\right) \geq-r
\end{array}
$$

integrando em relação a $r$, sobre $\left[s, R_{a}\right]$ e lembrando que $\lim _{s \rightarrow R_{a}^{-}} u_{a}(s)=+\infty$ e $\lim _{z \rightarrow \infty} F(z)=0$, temos

$$
\begin{gathered}
F\left(u_{a}\left(R_{a}\right)\right)-F\left(u_{a}(s)\right) \geq-\frac{\left(R_{a}^{2}-s^{2}\right)}{2} \Rightarrow \\
-F\left(u_{a}(s)\right) \geq-\frac{\left(R_{a}^{2}-s^{2}\right)}{2} \Rightarrow \\
F\left(u_{a}(s)\right) \leq \frac{\left(R_{a}^{2}-s^{2}\right)}{2} \leq R_{a}^{2}-s^{2} .
\end{gathered}
$$

Como $F^{\prime}(z)<0$ então $F$ é decrescente, isso produz 


$$
u_{a}(r) \geq F^{-1}\left(R_{a}^{2}-r^{2}\right)
$$

tomando $a \rightarrow A^{+}$temos

$$
u_{A}(r) \geq F^{-1}\left(R_{A}^{2}-r^{2}\right)
$$

Como $\lim _{z \rightarrow 0^{+}} F^{-1}(z)=+\infty$, pois quando $x \rightarrow \infty$ temos $F(x) \rightarrow 0$, então $\lim _{r \rightarrow R_{A}^{-}} u_{A}(r)=$ $+\infty$

Para completar a prova, escolha $\delta_{0}>0$ pequeno tal que $u_{A}(r)>M+1$ para $r>R_{A}-\delta_{0}$ (definição de limite). Observe que

$$
u_{A}\left(R_{A}-\frac{\delta_{0}}{2}\right)>M+1
$$

Usando (3.8) $\operatorname{com} \delta=\frac{\delta_{0}}{4}$ e $r=R_{A}-\frac{\delta_{0}}{2}$ temos

$$
u_{A}\left(R_{A}-\frac{\delta_{0}}{2}\right)-u_{a}\left(R_{A}-\frac{\delta_{0}}{2}\right)<(A-a) e^{\frac{M_{\delta}\left(R_{A}-\frac{\delta_{0}}{2}\right)^{2}}{2}}<(A-a) e^{\frac{M_{\delta} R_{A}^{2}}{2}}
$$

escolha $\varepsilon>0$ pequeno tal que $(A-a) e^{\lambda_{0} R_{A}^{2}}<1$, onde $\lambda_{0}=\frac{M_{\delta}}{2}$, para $0<A-a<\varepsilon$, então

$$
u_{A}\left(R_{A}-\frac{\delta_{0}}{2}\right)-u_{a}\left(R_{A}-\frac{\delta_{0}}{2}\right)<1
$$

onde $\lambda_{0}$ depende de $\delta_{0}$. Portanto de (3.10)

$$
\begin{aligned}
& u_{a}\left(R_{A}-\frac{\delta_{0}}{2}\right)-u_{A}\left(R_{A}-\frac{\delta_{0}}{2}\right)>-1 \Rightarrow \\
& u_{a}\left(R_{A}-\frac{\delta_{0}}{2}\right)>u_{A}\left(R_{A}-\frac{\delta_{0}}{2}\right)-1>M+1-1=M .
\end{aligned}
$$

Portanto $u_{a}(R)>M$ pois $R_{A}-\frac{\delta_{0}}{2} \leq R_{A} \leq R$ e $u_{a}$ é crescente. Então para algum $a$ próximo de $A$ temos $u_{a}(R)>M$.

Lema 3.1.4 Suponha g satisfaz G1, G2,G3. Se u é uma solução de (3.3), para todo $r \geq 0$ tal que $u(R) \geq 1$ então para $r \geq R$ a solução u satisfaz

$$
u(r) \leq\left\{\begin{array}{lll}
\left(\frac{R u^{\prime}(R)}{N-2}+u(R)+r^{2}\right)^{\frac{1}{1-\beta}}, & \text { se } & 0<\beta<1 \\
u(R) \exp \left(\frac{R u^{\prime}(R)}{N-2}+\frac{r^{2}}{2 N}\right), & \text { se } \quad \beta=1 .
\end{array}\right.
$$


Prova: Usamos G1, G2 e G3 para garantir, pelo Lema (3.1.3), que existe $a>0$ tal que $u_{a}$ é uma solução de (3.3) para todo $r \geq 0$ e $u_{a}(R) \geq 1$.

Como visto na prova do Lema (3.1.2), podemos escrever

$$
u(r)=u(R)+\frac{R u^{\prime}(R)}{N-2}\left(1-\left(\frac{R}{r}\right)^{N-2}\right)+\int_{R}^{r} t^{1-N} \int_{R}^{t} s^{N-1} g(u(s), s) d s d t .
$$

Portanto para $r \geq R$ note que $u(r) \geq 1$, pois $u(R) \geq 1$ e $u$ é crescente, e de $\mathbf{G} 2$ temos

$$
\begin{aligned}
u^{\prime}(r) & =\frac{-R^{N-1} u^{\prime}(R)}{N-2}\left(r^{2-N}\right)^{\prime}+r^{1-N} \int_{R}^{r} s^{N-1} g(u(s), s) d s \\
& =\frac{R^{N-1} u^{\prime}(R)}{2-N}(2-N) r^{1-N}+r^{1-N} \int_{R}^{r} s^{N-1} g(u(s), s) d s \\
& \leq u^{\prime}(R)\left(\frac{R}{r}\right)^{N-1}+r^{1-N} \int_{R}^{r} s^{N-1} u(s)^{\beta} d s, \quad \text { pois } u(r) \geq 1 \text { para } r \geq R \\
& \leq u^{\prime}(R)\left(\frac{R}{r}\right)^{N-1}+r^{1-N} u(r)^{\beta} \int_{R}^{r} s^{N-1} d s \\
& =u^{\prime}(R)\left(\frac{R}{r}\right)^{N-1}+\left.u(r)^{\beta} r^{1-N} \frac{s^{N}}{N}\right|_{R} ^{r} \\
& =u^{\prime}(R)\left(\frac{R}{r}\right)^{N-1}+u(r)^{\beta} r^{1-N} \frac{r^{N}-R^{N}}{N} \\
& =u^{\prime}(R)\left(\frac{R}{r}\right)^{N-1}+u(r)^{\beta} \frac{r}{N} .
\end{aligned}
$$

Multiplicando os dois lados da inequação por $u^{-\beta}$ e lembrando que $u(r) \geq 1$ para todo $r \geq R$, temos

$$
\begin{aligned}
& u(r)^{-\beta} u^{\prime}(r) \leq u^{\prime}(R)\left(\frac{R}{r}\right)^{N-1} u(r)^{-\beta}+\frac{r}{N}, \quad \text { para } r \geq R \stackrel{u(r)^{-1} \leq 1}{\Rightarrow} \leq 1 \\
& u(r)^{-\beta} u^{\prime}(r) \leq u^{\prime}(R)\left(\frac{R}{r}\right)^{N-1}+\frac{r}{N}, \quad \text { para } r \geq R .
\end{aligned}
$$

Suponha que $0<\beta<1$. Integrando os dois lados de (3.12) no intervalo $(R, r)$ para qualquer $r>R$ temos

$$
\int_{R}^{r} u(s)^{-\beta} u^{\prime}(s) d s \leq \int_{R}^{r}\left(u^{\prime}(R)\left(\frac{R}{s}\right)^{N-1}+\frac{s}{N}\right) d s \quad \Rightarrow
$$




$$
\begin{aligned}
\left.\frac{u(s)^{1-\beta}}{1-\beta}\right|_{R} ^{r} & \leq R^{N-1} u^{\prime}(R) \int_{R}^{r} s^{1-N} d s+\frac{1}{N} \int_{R}^{r} s d s \Rightarrow \\
\frac{u(r)^{1-\beta}-u(R)^{1-\beta}}{1-\beta} & \leq\left. R^{N-1} u^{\prime}(R) \frac{s^{2-N}}{2-N}\right|_{R} ^{r}+\left.\frac{1}{N} \frac{s^{2}}{2}\right|_{R} ^{r} \Rightarrow \\
u(r)^{1-\beta}-u(R)^{1-\beta} & \leq(1-\beta)\left[R^{N-1} u^{\prime}(R) \frac{r^{2-N}-R^{2-N}}{2-N}+\frac{r^{2}-R^{2}}{2 N}\right] \\
& \leq R^{N-1} u^{\prime}(R) \frac{\left(R^{2-N}-r^{2-N}\right)}{N-2}+\frac{\left(r^{2}-R^{2}\right)}{2 N} \\
& \leq \frac{R u^{\prime}(R)}{N-2}+\frac{r^{2}}{2 N} .
\end{aligned}
$$

Consequentemente, temos

$$
\begin{aligned}
u(r)^{1-\beta} & \leq \frac{R u^{\prime}(R)}{N-2}+\frac{r^{2}}{2 N}+u(R)^{1-\beta} \Rightarrow \\
u(r)^{1-\beta} & \leq \frac{R u^{\prime}(R)}{N-2}+r^{2}+u(R) \Rightarrow \\
u(r) & \leq\left(\frac{R u^{\prime}(R)}{N-2}+r^{2}+u(R)\right)^{\frac{1}{1-\beta}}
\end{aligned}
$$

provando assim a primeira desigualdade de (3.11). Quando $\beta=1$, de (3.12) temos

$$
u(r)^{-1} u^{\prime}(r) \leq u^{\prime}(R)\left(\frac{R}{r}\right)^{N-1}+\frac{r}{N}, \quad r \geq R
$$

integrando no intervalo $(R, r)$, obtemos

$$
\begin{aligned}
\left.\ln u(s)\right|_{R} ^{r} & \leq \frac{u^{\prime}(R) R}{N-2}+\frac{r^{2}}{2 N} \Rightarrow \\
\ln u(r)-\ln u(R) & \leq \frac{u^{\prime}(R) R}{N-2}+\frac{r^{2}}{2 N} \Rightarrow \\
\ln u(r) & \leq \ln u(R)+\frac{u^{\prime}(R) R}{N-2}+\frac{r^{2}}{2 N} \Rightarrow \\
u(r) & \leq u(R) e^{\frac{u^{\prime}(R) R}{N-2}+\frac{r^{2}}{2 N}} .
\end{aligned}
$$

Provando assim, a segunda desigualdade de (3.11).

OBSERVAÇÃo 2: Note que para provar as desigualdades, usamos apenas G2, no caso $r \geq R$. As outras condições foram necessárias apenas para garantir a existência de solução 
para (3.3) em todo $r \geq 0$.

\subsection{Demonstração do teorema (3.2.1) e (3.2.2)}

Definimos a função $g_{*}$ e $g^{*}$ de $(0, \infty) \times[0, \infty) \rightarrow(0, \infty)$ por

$$
g_{*}(z, r):=\min \left\{z^{q_{*}(r)}, z^{q^{*}(r)}\right\} \quad g^{*}(z, r):=\max \left\{z^{q_{*}(r)}, z^{q^{*}(r)}\right\}
$$

onde

$$
q^{*}(r):=\max _{|x|=r} q(x) \quad q_{*}(r):=\min _{|x|=r} q(x)
$$

e $q$ é dado em (3.1). Nós requeremos que $q$ satisfaz uma ou as duas condições que seguem: Q1- $q: \mathbb{R}^{n} \rightarrow(0, \infty)$ é localmente Hölder contínua;

Q2-existe $R \geq 0$ tal que $q(x) \geq 1$ para $|x| \leq R$, e $0<q(x) \leq 1$ para $|x| \geq R$.

Quando $R=0$, a condição Q2 é entendida como $0<q(x) \leq 1$ em $\mathbb{R}^{n}$.

Lema 3.2.1 Suponha q satisfaz Q1 e Q2. Então as funções $g_{*}$ e $g^{*}$ definida em (3.13) satisfazem $\mathbf{G 1}, \mathbf{G 2}$ e $\mathbf{G 3}$.

Prova: Temos que $g_{*}(z, r)$ e $g^{*}(z, r)$ são contínuas em $(0, \infty) \times[0, \infty) \rightarrow(0, \infty)$, pois as funções $z^{q_{*}(r)}$ e $z^{q^{*}(r)}$ são contínuas.

- Primeiramente vamos mostrar G1. Seja $r$ fixado e tome $t_{1} \leq t_{2}$ então

$$
t_{1}^{q_{*}(r)} \leq t_{2}^{q_{*}(r)} \quad \text { e } \quad t_{1}^{q^{*}(r)} \leq t_{2}^{q^{*}(r)}
$$

$\operatorname{assim} \min \left\{t_{1}^{q_{*}(r)}, t_{1}^{q^{*}(r)}\right\} \leq \min \left\{t_{2}^{q_{*}(r)}, t_{2}^{q^{*}(r)}\right\}$ então $g_{*}\left(t_{1}, r\right) \leq g_{*}\left(t_{2}, r\right)$, assim, $g_{*}(t, r)$ é não decrescente em relação a primeira variável. Analogamente provamos para $g^{*}(t, r)$.

Agora fixe um $t$, vamos mostrar que existe um $c_{t}>0$ tal que $g_{*}(t, r) \geq c_{t}$. Para $R \geq 0$ temos, $q_{*}(r) \geq 1$, para $r \leq R$, mas como $q$ é contínua e estamos em um conjunto compacto, temos $1 \leq q_{*}(r) \leq M$ é limitada. Para $t \in(0,1]$ temos

$$
t^{q_{*}(r)}>t^{M}
$$

para $t \in[1, \infty)$ segue que

$$
t^{q_{*}(r)} \geq t \geq 1
$$


No caso $0<q_{*}(r) \leq 1 \leq M$ para $r \geq R$ segue que, se $t \in(0,1]$

$$
t^{q_{*}(r)} \geq t \geq t^{M}
$$

e se $t \in[1, \infty)$

$$
t^{q_{*}(r)}>1
$$

tome $c_{t}=\min \left\{t^{M}, 1\right\}$ então $t^{q_{*}(r)} \geq c_{t}$. Analogamente provamos $t^{q^{*}(r)} \geq \tilde{c}_{t}$, então $g_{*}(t, r)=\min \left\{t^{q^{*}(r)}, t^{q_{*}(r)}\right\} \geq d_{t}$, como $g^{*}(t, r)=\max \left\{t^{q^{*}(r)}, t^{q_{*}(r)}\right\} \geq g_{*}(t, r) \geq d_{t}$ temos que $g_{*}(t, r)$ e $g^{*}(t, r)$ satisfazem $\mathbf{G} \mathbf{1}$.

- Se $R=0$ e para $t \geq 1$, como $0<q(x) \leq 1$, temos

$$
q^{*}(r)=\max _{|x|=r} q(x) \leq 1, \quad q_{*}(r)=\min _{|x|=r} q(x)>0 \quad \text { e } \quad q^{*}(r)>q_{*}(r)
$$

então $t^{q_{*}(r)}<t^{q^{*}(r)} \Rightarrow g^{*}(t, r)=t^{q^{*}(r)} \operatorname{como} q^{*}(r) \leq 1$ temos $g^{*}(t, r)=t^{q^{*}(r)} \leq t$. Logo $g_{*}(t, r) \leq g^{*}(t, r) \leq t$. Assim $g_{*}$ e $g^{*}$ satisfazem G2 para $R=0$.

Suponha $R>0$ então de Q2 temos $q(x) \geq 1$ para $|x| \leq R$ logo para $0 \leq r \leq R$ obtemos que $q^{*}(r) \geq q_{*}(r) \geq 1$. Para $t \in(0,1]$ assim $g^{*}(t, r)=t^{q_{*}(r)} \leq t$. Para $t \in[1, \infty)$ temos $g^{*}(t, r)=t^{q^{*}(r)}$, seja $\zeta=\max \{q(x) ;|x| \leq R\} \geq 1$ e portanto $q^{*}(r)=\max _{|x|=r} q(x) \leq \zeta$ então $g^{*}(t, r)=t^{q^{*}(r)} \leq t^{\zeta}$ para $t \geq 1$. Defina

$$
f(t)=\left\{\begin{array}{l}
t, \quad \text { para } t \in(0,1] \\
t^{\zeta}, \quad \text { para } t \in[1, \infty)
\end{array}\right.
$$

$f(t)$ é crescente, contínua e

$$
\begin{aligned}
\int_{0}^{1} \frac{1}{f(t)} d t & =\int_{0}^{1} \frac{1}{t} d t=\left.\ln t\right|_{0} ^{1}=+\infty \\
\int_{1}^{\infty} \frac{1}{f(t)} d t & =\int_{1}^{\infty} \frac{1}{t^{\zeta}} d t=\left.\frac{t^{1-\zeta}}{1-\zeta}\right|_{1} ^{\infty}=0-\frac{1}{1-\zeta}=\frac{1}{\zeta-1}<\infty
\end{aligned}
$$

então $g^{*}(t, r) \leq f(t)$ para $0 \leq r \leq R$ e $t \geq 0$ e $g_{*}(t, r)$ também pois $g_{*}(t, r) \leq$ $g^{*}(t, r) \leq f(t)$ para $0 \leq r \leq R$ e $t \geq 0$.

Para $r \geq R$ e $t \geq 1$ de $\mathbf{Q} 2,0<q(x) \leq 1$ para $|x| \geq R$ então

$$
g^{*}(t, r)=\max \left\{t^{q^{*}(r)}, t^{q_{*}(r)}\right\}=t^{q^{*}(r)}
$$

$\operatorname{seja} \beta=\max \{q(x) /|x| \geq R\}$ então $0<\beta \leq 1 \operatorname{logo} \beta \geq q^{*}(r), \operatorname{assim} g^{*}(t, r) \leq t^{\beta}$. 
Então $g_{*}(t, r) \leq g^{*}(t, r) \leq t^{\beta}$. Assim $g_{*}$ e $g^{*}$ satisfazem G2.

- Para mostrar G3 tome $0 \leq r \leq R$. Usando as definições de $g_{*}$ e $g^{*}$ do Apêndice (E) temos

$$
\begin{aligned}
\left|g_{*}(s, r)-g_{*}(t, r)\right| & =\mid \frac{s^{q_{*}(r)}+s^{q^{*}(r)}}{2}-\frac{\left|s^{q_{*}(r)}-s^{q^{*}(r)}\right|}{2}-\left(\frac{t^{q_{*}(r)}+t^{q^{*}(r)}}{2}\right) \\
& +\frac{\left|t^{q_{*}(r)}-t^{q^{*}(r)}\right|}{2} \mid
\end{aligned}
$$

Para $0<s<t<1$ temos $s^{q_{*}(r)}>s^{q^{*}(r)}$ e $t^{q_{*}(r)}>t^{q^{*}(r)}$ então

$$
\begin{aligned}
\left|g_{*}(s, r)-g_{*}(t, r)\right| & =\mid \frac{s^{q_{*}(r)}+s^{q^{*}(r)}}{2}-\frac{\left(s^{q_{*}(r)}-s^{q^{*}(r)}\right)}{2}-\frac{\left(t^{q_{*}(r)}+t^{q^{*}(r)}\right)}{2} \\
& +\frac{t^{q_{*}(r)}-t^{q^{*}(r)}}{2}|=| s^{q^{*}(r)}-t^{q^{*}(r)} \mid .
\end{aligned}
$$

Para $0<s<1$ e $1 \leq t$ temos $s^{q_{*}(r)}>s^{q^{*}(r)}, t^{q^{*}(r)}>t^{q_{*}(r)}$ e $s^{q^{*}(r)}<s^{q_{*}(r)}<t^{q_{*}(r)}<$ $t^{q^{*}(r)}$ então

$$
\begin{aligned}
\left|g_{*}(s, r)-g_{*}(t, r)\right| & =\mid \frac{s^{q_{*}(r)}+s^{q^{*}(r)}}{2}-\frac{\left(s^{q_{*}(r)}-s^{q^{*}(r)}\right)}{2}-\frac{\left(t^{q_{*}(r)}+t^{q^{*}(r)}\right)}{2} \\
& +\frac{t^{q^{*}(r)}-t^{q_{*}(r)}}{2}|=| s^{q^{*}(r)}-t^{q_{*}(r)}|\leq| s^{q^{*}(r)}-t^{q^{*}(r)} \mid .
\end{aligned}
$$

Para $s \geq 1$ e $t \geq 1$ obtemos que $s^{q_{*}(r)} \leq s^{q^{*}(r)}$ e $t^{q^{*}(r)} \geq t^{q_{*}(r)}$ então

$$
\begin{aligned}
\left|g_{*}(s, r)-g_{*}(t, r)\right| & =\mid \frac{s^{q_{*}(r)}+s^{q^{*}(r)}}{2}-\frac{\left(s^{q^{*}(r)}-s^{q_{*}(r)}\right)}{2}-\frac{\left(t^{q_{*}(r)}+t^{q^{*}(r)}\right)}{2} \\
& +\frac{t^{q^{*}(r)}-t^{q_{*}(r)}}{2}|=| s^{q_{*}(r)}-t^{q_{*}(r)} \mid .
\end{aligned}
$$

Assim,

$$
\left|g_{*}(s, r)-g_{*}(t, r)\right| \leq \max \left\{\left|s^{q_{*}(r)}-t^{q_{*}(r)}\right|,\left|s^{q^{*}(r)}-t^{q^{*}(r)}\right|\right\}
$$

Se $\max \left\{\left|s^{q_{*}(r)}-t^{q_{*}(r)}\right|,\left|s^{q^{*}(r)}-t^{q^{*}(r)}\right|\right\}=\left|s^{q_{*}(r)}-t^{q_{*}(r)}\right|$ então usando o Teorema do valor médio existe $c \in[s, t]$ ou $c \in[t, s]$ tal que

$$
\left|s^{q_{*}(r)}-t^{q_{*}(r)}\right|=q_{*}(r) c^{q_{*}(r)-1}|s-t| \leq\left\{\begin{array}{l}
\zeta(s+1+t)^{\zeta-1}|s-t|, \\
\zeta(t+1+s)^{\zeta-1}|s-t|
\end{array}\right.
$$

ou se $\max \left\{\left|s^{q_{*}(r)}-t^{q_{*}(r)}\right|,\left|s^{q^{*}(r)}-t^{q^{*}(r)}\right|\right\}=\left|s^{q^{*}(r)}-t^{q^{*}(r)}\right|$ então usando Teorema do 
valor médio existe $c \in[s, t]$ ou $c \in[t, s]$ tal que

$$
\left|s^{q^{*}(r)}-t^{q^{*}(r)}\right|=q^{*}(r) c^{q^{*}(r)-1}|s-t| \leq\left\{\begin{array}{l}
\zeta(s+1+t)^{\zeta-1}|s-t| \\
\zeta(s+1+t)^{\zeta-1}|s-t|
\end{array}\right.
$$

então $\mu(t, s)=\zeta(s+1+t)^{\zeta-1}$ para $0 \leq r \leq R$, portanto provamos que $g_{*}$ satisfaz G3, analogamente provamos que $g^{*}$ satisfaz G3.

Pelos Lemas (3.1.1), (3.1.2), (3.1.3), (3.1.4) e (3.2.1) temos

Corolário 3.2.1 Suponha que q satisfaz as condições $\mathbf{Q 1}$ e $\mathbf{Q} 2$ e tomando g em (3.3) como sendo $g_{*}$ ou $g^{*}$ como definido em (3.13). Então

1. Se $R=0$, então para qualquer $a>0$ a equação (3.3) tem uma solução definida para todo $r \geq 0$ e $u(r) \rightarrow \infty \operatorname{com} r \rightarrow \infty$.

Prova: se $R=0,0<q(x) \leq 1$ em $\mathbb{R}^{n}$ pelo Lema (3.2.1) temos que $g_{*}(z, r)$ e $g^{*}(z, r)$ satisfazem G1 e G2. Pelo Lema (3.1.1) temos que para qualquer $a>0$, a equação (3.3) tem uma solução positiva $u$ definida para todo $r \geq 0$, mais ainda $u(r) \rightarrow \infty \operatorname{com} r \rightarrow \infty$.

2. Se $R>0$ e $M$ é qualquer constante positiva, então existe $a>0$ tal que (3.3) tem uma solução $u$ definida para todo $r \geq 0$ para o qual $u(R)>M$ e $u(r) \rightarrow \infty$ com $r \rightarrow \infty$.

Prova: Pelo Lema (3.2.1) temos que $g_{*}(z, r)$ e $g^{*}(z, r)$ satisfazem G1, G2 e G3, pelo Lema (3.1.3) temos que dado $M>0$ existe um $a>0$ tal que a solução inteira positiva de (3.3) correspondente de $a$, satisfaz $u_{a}(R)>M$. Como $u_{a}$ é solução de (3.3), temos

$$
u_{a}(r)=a+\int_{0}^{r} t^{1-N} \int_{0}^{t} s^{N-1} g_{*}(u(s), s) d s d t
$$

como $u_{a}(s)>a$ e $g_{*}$ é não decrescente na primeira variável, temos

$$
u_{a}(r) \geq a+\int_{0}^{r} t^{1-N} \int_{0}^{t} s^{N-1} g_{*}(a, s) d s d t \stackrel{G 1}{\geq} a+\frac{c_{a} r^{2}}{2 N}
$$

então quando $r \rightarrow+\infty$ temos $u_{a}(r) \rightarrow+\infty$. Analogamente provamos para $g=g^{*}$

3. Se $u$ é uma solução de (3.3) definida para todo $r \geq 0$ e satisfazendo $u(R) \geq 1$, então $u$ satisfaz (3.11) para $r \geq R$. 
Prova: Do Lema (3.2.1) temos $g_{*}$ e $g^{*}$ satisfazem G1, G2 e G3, portanto pelo Lema (3.1.4) temos se $u$ é solução de (3.3) para todo $r \geq 0$ tal que $u(R) \geq 1$, então para $r \geq R$ a solução $u$ satisfaz (3.11).

O próximo resultado mostra que (3.1) tem infinitas soluções, para $q$ satisfazendo algumas condições.

Teorema 3.2.1 Suponha q satisfaz condições $\mathbf{Q 1}$ e Q2. Se

$$
\int_{0}^{r} r \exp \left(\lambda r^{2}\right) q_{o s c}(r) d r<\infty
$$

para algum $\lambda$ tal que $2 N \lambda>1$ então o problema (3.1) admite infinitas soluções.

Prova: Considere as equações

$$
\triangle v=g^{*}(v(r), r) \quad \text { e } \quad \triangle w=g_{*}(w(r), r), \quad \text { em } \mathbb{R}^{n}
$$

cujas funções $g_{*}$ e $g^{*}$ são definidas em (3.13). Dado as constantes positivas $a$ e $b$, então $v(x):=v_{a}(|x|)$ e $w(x):=w_{b}(|x|)$, onde $v_{a}$ e $w_{b}$ são soluções da equação integral (3.3) com $v_{a}(0)=a$ e $w_{b}(0)=b$ respectivamente. Para $M=1$ no Corolário (3.2.1), a condição 2 garante a existência de um $a>0$ tal que $v_{a}$ é solução de (3.3) para todo $r \geq 0$ e, $v_{a}(R) \geq 1$.

Mostraremos que $b$ pode ser escolhido tal que $v_{a} \leq w_{b}$ em $(0, \infty)$. A partir deste ponto, não usaremos $v_{a}$ nem $w_{b}$, iremos suprimir os índices, mas lembre-se o $a$ já foi escolhido. Observe que $\beta=\sup \{q(x) ;|x| \geq R\}=1$, pois para $|x| \geq R$ temos $0<q(x) \leq 1$. Usando a condição 3 do Corolário (3.2.1) $\operatorname{com} \beta=1$, temos

$$
v(r) \leq \zeta(r) \quad \text { com } \quad r \geq R
$$

onde definimos $\zeta(r):=v(R) e^{\frac{R v^{\prime}(R)}{N-2}+\frac{r^{2}}{2 N}}$.

Seja $R_{0}:=\sup \{r>0 ; v(t) \leq w(t)$ para todo $0 \leq t \leq r\}$.

Pelo Corolário (3.2.1) condição 2, escolha $b$ tal que

$$
w_{b}(R)>v(R)+\frac{R v^{\prime}(R)}{N-2}+\int_{0}^{\infty} r q_{o s c}(r) \zeta(r) \log \zeta(r) d r
$$

a integral do lado direito de (3.17) é finita pelo que foi visto no Apêndice F, então $v(R)<w(R)$ e $g^{*}(z, r) \geq g_{*}(z, r)$ usando o princípio de comparação temos que 


$$
v(r) \leq w(r), \quad \text { para todo } 0 \leq r \leq R
$$

então $R_{0}>R$. Se $R_{0}=+\infty$ então $v \leq w$ para todo $r \geq 0$. Suponha que $R_{0}<\infty$. Então como $v(r) \geq 1$ para todo $r \geq R$, (pois $v(R) \geq 1$ e $v$ é não decrescente então $v(r) \geq 1$ para todo $r \geq R$ ) usando os mesmos cálculos do Lema (3.1.2) e o Apêndice E, temos

$$
\begin{aligned}
v\left(R_{0}\right) & =a+\int_{0}^{R_{0}} t^{1-N} \int_{0}^{t} s^{N-1} g^{*}(v(s), s) d s d t \\
& =v(R)+\frac{R v^{\prime}(R)}{N-2}\left(1-\left(\frac{R}{R_{0}}\right)^{N-2}\right)+\int_{R}^{R_{0}} t^{1-N} \int_{R}^{t} s^{N-1} g^{*}(v(s), s) d s d t \\
& =v(R)+\frac{R v^{\prime}(R)}{N-2}\left(1-\left(\frac{R}{R_{0}}\right)^{N-2}\right)+\int_{R}^{R_{0}} t^{1-N} \int_{R}^{t} s^{N-1} \frac{v(s)^{q_{*}(s)}+v(s)^{q^{*}(s)}}{2} d s d t \\
& +\int_{R}^{R_{0}} t^{1-N} \int_{R}^{t} s^{N-1} \frac{v(s)^{q^{*}(s)}-v(s)^{q_{*}(s)}}{2} d s d t
\end{aligned}
$$

pois

$$
\begin{aligned}
s \geq R \Rightarrow v(s) \geq 1 \text { e } q_{*}(s) & \leq q^{*}(s) \text { então } v(s)^{q^{*}(s)}>v(s)^{q_{*}(s)} \Rightarrow \\
\left|v(s)^{q_{*}(s)}-v(s)^{q^{*}(s)}\right| & =v(s)^{q^{*}(s)}-v(s)^{q_{*}(s)} .
\end{aligned}
$$

Então

$$
\begin{aligned}
v\left(R_{0}\right) & =v(R)+\frac{R v^{\prime}(R)}{N-2}\left(1-\left(\frac{R}{R_{0}}\right)^{N-2}\right)+\int_{R}^{R_{0}} t^{1-N} \int_{R}^{t} s^{N-1} v(s)^{q^{*}(s)} d s d t \\
& =v(R)+\frac{R v^{\prime}(R)}{N-2}\left(1-\left(\frac{R}{R_{0}}\right)^{N-2}\right)+\int_{R}^{R_{0}} t^{1-N} \int_{R}^{t} s^{N-1}\left[v(s)^{q^{*}(s)}-v(s)^{q_{*}(s)}\right] d s d t \\
& +\int_{R}^{R_{0}} t^{1-N} \int_{R}^{t} s^{N-1} v(s)^{q_{*}(s)} d s d t .
\end{aligned}
$$

Usando a hipótese $0<q(x) \leq 1$ para $|x| \geq R$ e o teorema do valor médio, defina $f(x)=c^{x}$ para $c \geq 1$ e $0 \leq x \leq 1$ e $f^{\prime}(x)=c^{x} \log c$. Então

$$
c^{\gamma}-c^{\alpha}=c^{\theta} \log c(\gamma-\alpha) \leq c \log c(\gamma-\alpha)
$$

para $0<\alpha<\theta<\gamma<1$. 
Assim

$\int_{R}^{R_{0}} t^{1-N} \int_{R}^{t} s^{N-1}\left[v(s)^{q^{*}(s)}-v(s)^{q_{*}(s)}\right] d s d t \leq \int_{R}^{R_{0}} t^{1-N} \int_{R}^{t} s^{N-1} v(s) \log v(s) q_{\text {osc }}(s) d s d t$

onde $q_{\text {osc }}(s)=q^{*}(s)-q_{*}(s)$. Usando (3.19) e o fato de $1 \leq v(s) \leq w(s), \quad \forall s<R_{0}$ temos

$$
\begin{aligned}
v\left(R_{0}\right) & \leq v(R)+\frac{R v^{\prime}(R)}{N-2}\left(1-\left(\frac{R}{R_{0}}\right)^{N-2}\right)+\int_{R}^{R_{0}} t^{1-N} \int_{R}^{t} s^{N-1} v(s) \log v(s) q_{o s c}(s) d s d t \\
& +\int_{R}^{R_{0}} t^{1-N} \int_{R}^{t} s^{N-1} w(s)^{q_{*}(s)} d s d t \\
& =v^{*}(R)+\int_{R}^{R_{0}} t^{1-N} \int_{R}^{t} s^{N-1} v(s) \log v(s) q_{o s c}(s) d s d t+\int_{R}^{R_{0}} t^{1-N} \int_{R}^{t} s^{N-1} w(s)^{q_{*}(s)} d s d t
\end{aligned}
$$

onde $v^{*}(R)=v(R)+\frac{R v^{\prime}(R)}{N-2}$. Usando (3.16) e integração por partes, temos

$$
\begin{aligned}
& \int_{R}^{R_{0}} t^{1-N} \int_{R}^{t} s^{N-1} v(s) \log v(s) q_{\text {osc }}(s) d s d t \leq \int_{R}^{R_{0}} t^{1-N} \int_{R}^{t} s^{N-1} \zeta(s) \log \zeta(s) q_{\text {osc }}(s) d s d t \\
& =\frac{R_{0}^{2-N}}{2-N} \int_{R}^{R_{0}} s^{N-1} q_{\text {osc }}(s) \zeta(s) \log \zeta(s) d s-\int_{R}^{R_{0}} \frac{t}{2-N} q_{\text {osc }}(t) \zeta(t) \log \zeta(t) d t .
\end{aligned}
$$

Mas a primeira integral depois da igualdade (3.20) é negativa, portanto

$$
\begin{aligned}
\int_{R}^{R_{0}} t^{1-N} \int_{R}^{t} s^{N-1} v(s) \log v(s) q_{o s c}(s) d s d t & \leq \int_{R}^{R_{0}} \frac{t}{N-2} q_{o s c}(t) \zeta(t) \log \zeta(t) d t \\
& \leq \int_{R}^{R_{0}} t q_{o s c}(t) \zeta(t) \log \zeta(t) d t \\
& \leq \int_{0}^{\infty} t q_{o s c}(t) \zeta(t) \log \zeta(t) d t
\end{aligned}
$$

Logo 


$$
\begin{aligned}
v\left(R_{0}\right) & \leq v^{*}(R)+\int_{0}^{\infty} t q_{o s c}(t) \zeta(t) \log \zeta(t) d t+\int_{R}^{R_{0}} t^{1-N} \int_{R}^{t} s^{N-1} w(s)^{q_{*}(s)} d s d t \\
& <w(R)+\frac{R w^{\prime}(R)}{N-2}\left(1-\left(\frac{R}{R_{0}}\right)^{N-2}\right)+\int_{R}^{R_{0}} t^{1-N} \int_{R}^{t} s^{N-1} w(s)^{q_{*}(s)} d s d t \\
& =w\left(R_{0}\right)
\end{aligned}
$$

observando que $g_{*}(w(s), s)=w(s)^{q_{*}(s)}$, pois para $R \leq s$ temos

$$
\begin{gathered}
1 \leq v(R) \leq w(R) \leq w(s) \Rightarrow w(s) \geq 1 \text { e } q_{*}(s) \leq q^{*}(s) \Rightarrow w(s)^{q_{*}(s)} \leq w(s)^{q^{*}(s)} \\
\Rightarrow g_{*}(w(s), s)=w(s)^{q_{*}(s)}
\end{gathered}
$$

Então temos $v\left(R_{0}\right)<w\left(R_{0}\right)$ que contradiz a definição de $R_{0}$. Portanto $v \leq w$ em $\mathbb{R}^{n} \mathrm{e}$

- se $g^{*}(v(r), r)=\max \left\{v(r)^{q_{*}(r)}, v(r)^{q^{*}(r)}\right\}=v(r)^{q_{*}(r)}$ então $v(r) \leq 1$. Como $q_{*}(r) \leq$ $q(x)$ temos que $v(r)^{q_{*}(r)} \geq v(r)^{q(x)} \operatorname{assim} \Delta v \geq v(r)^{q(x)}$.

- Se $g^{*}(v(r), r)=\max \left\{v(r)^{q_{*}(r)}, v(r)^{q^{*}(r)}\right\}=v(r)^{q^{*}(r)}$ então $v(r) \geq 1$. Como $q^{*}(r) \geq$ $q(x)$ temos que $v(r)^{q(x)} \leq v(r)^{q^{*}(r)} \operatorname{assim} \Delta v \geq v(r)^{q(x)}$.

- Se $g_{*}(w(r), r)=\min \left\{w(r)^{q_{*}(r)}, w(r)^{q^{*}(r)}\right\}=w(r)^{q_{*}(r)}$ então $w(r) \geq 1$. Como $q_{*}(r) \leq$ $q(x)$ temos que $w(r)^{q_{*}(r)} \leq w(r)^{q(x)} \operatorname{assim} \Delta w \leq w(r)^{q(x)}$.

- Se $g^{*}(w(r), r)=\min \left\{w(r)^{q_{*}(r)}, w(r)^{q^{*}(r)}\right\}=w(r)^{q^{*}(r)}$ então $w(r) \leq 1$. Como $q^{*}(r) \geq$ $q(x)$ temos que $w(r)^{q^{*}(r)} \leq w(r)^{q(x)} \operatorname{assim} \Delta w \leq w(r)^{q(x)}$.

Então $v$ é subsolução de (3.1) e $v \leq w$, do Teorema 2.10 de [14] existe a solução de (3.1), $u$, tal que $v_{a} \leq u_{a} \leq w_{b}$, escolhendo $a^{\prime}>0$ pela condição 2 do Corolário (3.2.1), tal que $v_{a^{\prime}}(R)>u_{a}(R) \geq v_{a}(R) \geq 1$ então como na prova anterior existe $b^{\prime}>0$ tal que $v_{a^{\prime}} \leq w_{b^{\prime}}$, tal que

$$
\triangle v_{a^{\prime}} \geq v_{a^{\prime}}^{q(x)} \quad \text { e } \quad \triangle w_{b^{\prime}} \leq w_{b^{\prime}}^{q(x)}
$$

então existe uma solução de (3.1) $u_{a^{\prime}}$ tal que

$$
v_{a^{\prime}} \leq u_{a^{\prime}} \leq w_{b^{\prime}}
$$

Observe que $u_{a} \neq u_{a^{\prime}}$ pois

$$
u_{a}(R)<v_{a^{\prime}}(R) \leq u_{a^{\prime}}(R)
$$


Procedendo dessa maneira encontramos infinitas soluções.

Nossos próximos resultados nos mostram que para $R>0$ suficientemente pequeno, podemos permitir $1-q(x)$ mudar de sinal em $|x| \leq R$ e ainda assim obter existência da solução para (3.1). Para provarmos isso, precisamos do lema sobre a existência de solução para $(3.2) \operatorname{com} g(v(r), r)=v(r)^{q^{*}(r)}$ isto é

$$
\left\{\begin{aligned}
\left(r^{N-1} v^{\prime}\right)^{\prime} & =r^{N-1} v^{q^{*}(r)}, \quad r>0 \\
v(0) & =a \\
v^{\prime}(0) & =0
\end{aligned}\right.
$$

No lema abaixo vamos assumir que $q \in L^{\infty}\left(\mathbb{R}^{n}\right)$ e usar a notação $\|q\|_{\infty}:=\max \{q(x) ; x \in$ $\left.\mathbb{R}^{n}\right\}$.

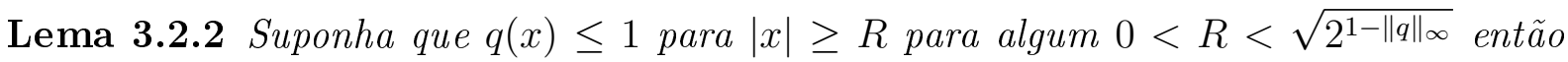
o problema (3.21) admite uma solução $v_{a} \geq$ a para algum $a \geq 1$, e $\lim _{r \rightarrow \infty} v_{a}(r)=+\infty$. Em particular, se $0<\|q\|_{\infty} \leq 1$ o problema (3.21) admite tal solução $v_{a}$ para qualquer $a \geq 1$.

Prova: Observe que se $0<\|q\|_{\infty} \leq 1$ então $0 \leq 1-\|q\|_{\infty}<1$ e para qualquer $a \geq 1$ temos $2 a \geq 2 \Rightarrow(2 a)^{1-\|q\|_{\infty}} \geq 2^{1-\|q\|_{\infty}}$ então $0<R<\sqrt{(2 a)^{1-\|q\|_{\infty}}}$ para qualquer $a \geq 1$. Fixe $a \geq 1$ tal que $0<R<\sqrt{(2 a)^{1-\|q\|_{\infty}}}$.

Seja $v_{0}=a$ e defina a sequência $\left\{v_{j}\right\}$ indutivamente como segue:

$$
v_{j}(r)=a+\int_{0}^{r} t^{1-N} \int_{0}^{t} s^{N-1}\left(v_{j-1}(s)\right)^{q^{*}(s)} d s d t \quad r \geq 0
$$

Escolha $r_{0}$ tal que

$$
R<r_{0}<\sqrt{(2 a)^{1-\|q\|_{\infty}}}
$$

Então, por indução, mostraremos que $\mathrm{a} \leq v_{j} \leq 2 a \quad r \in\left[0, r_{0}\right], \quad j=0,1,2,3, \ldots$. Como $v_{0}=a$ e $v_{j}$ é crescente então $v_{j} \geq a$ para $j=1,2,3, \ldots$ Para $j=1$, temos 


$$
\begin{aligned}
\int_{0}^{r} t^{1-N} \int_{0}^{t} s^{N-1}\left(v_{0}(s)\right)^{q^{*}(s)} d s d t & =\int_{0}^{r} t^{1-N} \int_{0}^{t} s^{N-1} a^{q^{*}(s)} d s d t \\
& \leq \int_{0}^{r} t^{1-N} \int_{0}^{t} s^{N-1} a^{\|q\|_{\infty}} d s d t, \quad \text { pois } q^{*}(s) \leq\|q\|_{\infty} \\
& =\left.\int_{0}^{r} t^{1-N} a^{\|q\|_{\infty}} \frac{s^{N}}{N}\right|_{0} ^{t} d t \\
& =\int_{0}^{r} t^{1-N} a^{\|q\|_{\infty}} \frac{t^{N}}{N} d t \\
& \leq \int_{0}^{r} t a^{\|q\|_{\infty}} d t \\
& =\left.a^{\|q\|_{\infty}} \frac{t^{2}}{2}\right|_{0} ^{r}=a^{\|q\|_{\infty}} \frac{r^{2}}{2} \\
& =a^{\|q\|_{\infty}} \frac{(2 a)^{1-\|q\|_{\infty}}}{2}=a 2^{-\|q\|_{\infty}} \leq a, \text { pois } r<\sqrt{(2 a)^{1-\|q\|_{\infty}}}
\end{aligned}
$$

Assim $v_{1}(r) \leq a+a=2 a$. Suponha que $j=k$ temos $a \leq v_{k} \leq 2 a$ então

$$
\begin{aligned}
v_{k+1}(r) & =a+\int_{0}^{r} t^{1-N} \int_{0}^{t} s^{N-1}\left(v_{k}(s)\right)^{q^{*}(s)} d s d t \\
& \leq a+\int_{0}^{r} t^{1-N} \int_{0}^{t} s^{N-1}(2 a)^{q^{*}(s)} d s d t, \quad \text { já que } 1 \leq v_{k}(s) \leq 2 a \\
& \leq a+\int_{0}^{r} t^{1-N} \int_{0}^{t} s^{N-1}(2 a)^{\|q\|_{\infty}} d s d t, \quad \text { pois }\|q\|_{\infty}>q^{*}(s) \\
& =a+\int_{0}^{r} t^{1-N}(2 a)^{\|q\|_{\infty}} \frac{t^{N}}{N} d t \\
& =a+\int_{0}^{r}(2 a)^{\|q\|_{\infty}} \frac{t}{N} d t \\
& \leq a+(2 a)^{\|q\|_{\infty}} \frac{r^{2}}{2} \\
& <a+(2 a)^{\|q\|_{\infty}} \frac{(2 a)^{1-\|q\|_{\infty}}}{2} \\
& =a+a=2 a .
\end{aligned}
$$

Então por indução mostramos que $a \leq v_{j}(r) \leq 2 a$ para todo $r \in\left[0, r_{0}\right]$ e $j=0,1,2,3, \ldots$. Observe também que $v_{j} \leq v_{j+1}$ em $\left[0, r_{0}\right]$, também provamos isso por indução. De fato,

$$
v_{0}=a \leq v_{1}
$$


Suponha $v_{n} \leq v_{n+1} \operatorname{como} v_{n} \geq a$ e $a \geq 1$ temos $v_{n} \geq 1$, assim

$$
\begin{aligned}
v_{n+2}(r) & =a+\int_{0}^{r} t^{1-N} \int_{0}^{t} s^{N-1}\left(v_{n+1}(s)\right)^{q^{*}(s)} d s d t \\
& \geq a+\int_{0}^{r} t^{1-N} \int_{0}^{t} s^{N-1}\left(v_{n}(s)\right)^{q^{*}(s)} d s d t=v_{n+1}(r)
\end{aligned}
$$

$\operatorname{assim} v_{j} \leq v_{j+1}$ em $\left[0, r_{0}\right]$. Consequentemente $v_{j}$ converge para um $v$ em $\left[0, r_{0}\right]$ e $v$ satisfaz

$$
v(r)=a+\int_{0}^{r} t^{1-N} \int_{0}^{t} s^{N-1}(v(s))^{q^{*}(s)} d s d t, \quad r \in\left[0, r_{0}\right]
$$

então $v$ satisfaz (3.21). Seja $\left[0, r_{1}\right]$ para algum $r_{1} \geq r_{0}$ o intervalo maximal de existência de $v$. Vamos mostrar que $r_{1}=+\infty$. Suponhamos que $v(r) \rightarrow+\infty \operatorname{com} r \rightarrow r_{1}^{-}$(pois se $v$ limitada em $\left[0, r_{1}\right]$, então

$$
\begin{aligned}
v^{\prime}(r) & =r^{1-N} \int_{0}^{r} s^{N-1}(v(s))^{q^{*}(s)} d s \leq r^{1-N} \int_{0}^{r} s^{N-1}(2 a)^{\|q\|_{\infty}} d s \\
& =r^{1-N}(2 a)^{\|q\|_{\infty}} \frac{r^{N}}{N} \leq r(2 a)^{\|q\|_{\infty}} \leq r_{1}(2 a)^{\|q\|_{\infty}}
\end{aligned}
$$

logo $v^{\prime}$ é limitada em $\left[0, r_{1}\right]$ então podemos estender a função $v$ além de $\left[0, r_{1}\right]$ o que é absurdo então $\left.\lim _{r \rightarrow r_{1}^{-}} v(r)=+\infty\right)$. Como $q^{*}(|x|) \leq 1$ para $R \leq|x| \leq r_{1}$ (pois $q(x) \leq 1$ para $|x| \geq R$ ) temos que $v$ é solução do problema

$$
\triangle v=v^{q^{*}(|x|)}, \quad x \in B\left(0, r_{1}\right)
$$

mas pelo Teorema 1 de [6] temos que o problema (3.22) não tem solução contradição, então $r_{1}=+\infty$ logo o problema (3.21) admite solução para algum $a \geq 1$. E para $0<\|q\|_{\infty} \leq 1$ e qualquer $a \geq 1$ temos (3.21) tem solução.

Para o próximo teorema, vamos considerar a seguinte notação. Dado $0<\beta \leq 1$, temos

$$
v_{\beta}(r):= \begin{cases}r^{\frac{2}{1-\beta}}, & \text { se } 0<\beta<1 \\ \exp \left(r^{2}\right), & \text { se } \beta=1\end{cases}
$$

Teorema 3.2.2 Se q satisfaz $Q 1$ e suponha que existem constantes $0<R<\sqrt{2^{1-\|q\|_{\infty}}}$ e $0<\beta \leq 1$ tal que $0 \leq q(x) \leq \beta$ para todo $|x| \geq R$. Mais ainda, assuma que $q_{*}(r) \leq 1$ 
para $r>0 . S e$

$$
\int_{1}^{\infty} r v_{\beta}(r) q_{o s c}(r) d r<\infty
$$

então o problema (3.1) admite uma solução positiva.

Prova: Considere as equações

$$
\begin{array}{r}
\Delta v=v^{q^{*}(r)} \quad e \\
\triangle w=w^{q_{*}(r)}, \quad \text { em } \mathbb{R}^{n}
\end{array}
$$

pelo Lema (3.2.2) fixe $a \geq 1$ tal que $R<\sqrt{(2 a)^{1-\|q\|_{\infty}}}$. Então pelo Lema (3.2.2) o problema (3.21) admite solução $v_{a} \geq a$ para $a \geq 1$ e $\lim _{r \rightarrow \infty} v_{a}(r)=+\infty$. Então $v(x)=$ $v_{a}(|x|)$, onde $v_{a}$ é uma solução do problema (3.21), é uma solução de (3.24)

$$
v_{a}(r)=a+\int_{0}^{r} t^{1-N} \int_{0}^{t} s^{N-1}\left(v_{a}(s)\right)^{q^{*}(s)} d s d t \quad r \geq 0 .
$$

Assuma que $0<\beta<1$. Como $g\left(v_{a}(s), s\right)=\left(v_{a}(s)\right)^{q^{*}(s)}$ vamos mostrar que essa função satisfaz G2. Para $|x| \geq R$ temos $0 \leq q(x) \leq \beta$ então $q^{*}(|x|) \leq \beta$, como $v_{a} \geq a \geq 1$ podemos concluir que $v_{a}^{q^{*}(|x|)} \leq v_{a}^{\beta}$. Como $g\left(v_{a}(s), s\right)$ satisfaz G2 para o caso $s \geq R$ temos da Observação 2 do Lema (3.1.4) que

$$
v(r) \leq \varpi(r) \quad r \geq R
$$

onde

$$
\varpi(r)=\left(\frac{R v^{\prime}(R)}{N-2}+v(R)+r^{2}\right)^{\frac{1}{1-\beta}}, \quad r \geq 0, \quad 0<\beta<1 .
$$

Agora vamos garantir a existência de uma solução $w_{b}$ para (3.25). Para $R=0$ a função $g\left(w_{b}(s), s\right)=w_{b}^{q_{*}(s)}$ satisfaz $\mathbf{G 2}$, pois como $q_{*}(r) \leq 1$ para $r \geq 0$ e para $b \geq 1$ temos $w_{b} \geq 1$ então $w_{b}^{q_{*}(r)} \leq w_{b}^{1}$. E além disso, a função $g\left(w_{b}(s), s\right)=w_{b}^{q_{*}(s)}$ satisfaz $\mathbf{G 1}$, pois se $z_{1} \leq z_{2} \Rightarrow z_{1}^{q_{*}(r)} \leq z_{2}^{q_{*}(r)}$ então $g(z, r)=z^{q_{*}(r)}$ é não decrescente na primeira coordenada e $w_{b}(s) \geq 1$ então $w_{b}(s)^{q_{*}(s)} \geq 1$. Assim, pelo Lema (3.1.1) temos que para qualquer $b \geq 1$ existe uma solução $w_{b}$ definida por:

$$
w_{b}(r)=b+\int_{0}^{r} t^{1-N} \int_{0}^{t} s^{N-1} w_{b}(s)^{q_{*}(s)} d s d t .
$$


Vamos mostrar que $b$ pode ser escolhido de tal forma que $1 \leq v_{a} \leq w_{b}$ em $(0, \infty)$. Essa prova é semelhante a prova do teorema (3.2.1).

Como $0<\beta<1$, observe que $\varpi^{\beta}(r) \log \varpi(r) \leq \varpi(r)$. De fato, seja $f(x)=x^{\beta} \log x-$ $x=x\left(x^{\beta-1} \log x-1\right)=x\left(\frac{\log x}{x^{1-\beta}}-1\right)$ então para $x$ suficientemente grande

$$
\lim _{x \rightarrow \infty} \frac{\log x}{x^{1-\beta}}=\lim _{x \rightarrow \infty} \frac{\frac{1}{x}}{(1-\beta) x^{-\beta}}=\lim _{x \rightarrow \infty} \frac{1}{(1-\beta) x^{1-\beta}}=0
$$

e

$$
\lim _{x \rightarrow \infty} f(x)=\lim _{x \rightarrow \infty} x\left(\frac{\log x}{x^{1-\beta}}-1\right)=-\infty<0
$$

ou seja, para todo $N>0$ existe $M>0$ tal que se $x>M$ então $f(x)<-N$. Assim para $x$ suficientemente grande $f(x) \leq 0 \operatorname{logo} x^{\beta} \log x \leq x$. Ou seja, para $r>C$ temos $\varpi(r) \geq M$ então $\varpi(r)^{\beta} \log \varpi(r) \leq \varpi(r)$. E

- para $C \geq 1$ temos

$$
\begin{aligned}
\int_{0}^{\infty} r q_{o s c}(r) \varpi^{\beta}(r) \log \varpi(r) d r & \leq \int_{0}^{C} r q_{o s c}(r) \varpi^{\beta}(r) \log \varpi(r) d r+\int_{C}^{\infty} r q_{o s c}(r) \varpi(r) d r \\
& \leq K_{1}+\int_{C}^{\infty} r q_{o s c}(r) \varpi(r) d r .
\end{aligned}
$$

Como para $r \geq 1$ existe $c>0$ tal que

$$
\begin{aligned}
\frac{R v^{\prime}(R)}{N-2}+v(R) \leq c r^{2} & \Rightarrow \frac{R v^{\prime}(R)}{N-2}+v(R)+r^{2} \leq(c+1) r^{2} \Rightarrow \\
\left(\frac{R v^{\prime}(R)}{N-2}+v(R)+r^{2}\right)^{\frac{1}{1-\beta}} & \leq\left((c+1) r^{2}\right)^{\frac{1}{1-\beta}}=K r^{\frac{2}{1-\beta}} .
\end{aligned}
$$

Usando a hipótese (3.23) concluímos que a integral

$$
\int_{0}^{\infty} r q_{o s c}(r) \varpi^{\beta}(r) \log \varpi(r) d r \leq K_{1}+\int_{C}^{\infty} r q_{o s c}(r) K r^{\frac{2}{1-\beta}} d r<\infty .
$$

é finita.

- para $C \leq 1$ temos

$$
\begin{aligned}
\int_{0}^{\infty} r q_{o s c}(r) \varpi^{\beta}(r) \log \varpi(r) d r & \leq K_{2}+\int_{C}^{1} r q_{o s c}(r) \varpi(r) d r+\int_{1}^{\infty} r q_{o s c}(r) \varpi(r) d r \\
& \leq K_{3}+\int_{1}^{\infty} r q_{o s c}(r) \varpi(r) d r .
\end{aligned}
$$


Como para $r \geq 1$ existe $c>0$ tal que

$$
\begin{aligned}
\frac{R v^{\prime}(R)}{N-2}+v(R) \leq c r^{2} & \Rightarrow \frac{R v^{\prime}(R)}{N-2}+v(R)+r^{2} \leq(c+1) r^{2} \Rightarrow \\
\left(\frac{R v^{\prime}(R)}{N-2}+v(R)+r^{2}\right)^{\frac{1}{1-\beta}} & \leq\left((c+1) r^{2}\right)^{\frac{1}{1-\beta}}=K r^{\frac{2}{1-\beta}} .
\end{aligned}
$$

Usando a hipótese (3.23) concluímos que a integral

$$
\int_{0}^{\infty} r q_{o s c}(r) \varpi^{\beta}(r) \log \varpi(r) d r \leq K_{3}+\int_{1}^{\infty} r q_{o s c}(r) K r^{\frac{2}{1-\beta}} d r<\infty .
$$

é finita.

Assim, tome $b$ tal que

$$
b>v(R)+\frac{R v^{\prime}(R)}{N-2}+\frac{1}{N-2} \int_{0}^{\infty} r q_{o s c}(r) \varpi^{\beta}(r) \log \varpi(r) d r .
$$

Como de (3.27)

$$
w_{b}(R)>b>v(R)+\frac{R v^{\prime}(R)}{N-2}+\frac{1}{N-2} \int_{0}^{\infty} r q_{o s c}(r) \varpi^{\beta}(r) \log \varpi(r) d r>v(R)
$$

temos que $w_{b}(R)>v_{a}(R) \geq 1$ e $g(z, r)=z^{q^{*}(r)}>z^{q_{*}(r)}$ para $z \geq 1$ usando o princípio da comparação $v_{a}(r)<w_{b}(r)$ para $0 \leq r \leq R$.

Defina $R_{0}:=\sup \left\{r>0 / v_{a}(t) \leq w_{b}(t)\right.$ para $\left.0 \leq t \leq r\right\}$. Como $v_{a}(R)<w_{b}(R)$ temos $R<R_{0}$. Se $R_{0}=\infty$ então $v_{a} \leq w_{b}$ para $r \geq 0$. Suponha que $R_{0}<\infty$ então

$$
\begin{aligned}
v\left(R_{0}\right) & =a+\int_{0}^{R_{0}} t^{1-N} \int_{0}^{t} s^{N-1} v_{a}^{q^{*}(s)} d s d t \\
& =v(R)+\frac{R v^{\prime}(R)}{N-2}\left(1-\left(\frac{R}{r}\right)^{N-2}\right)+\int_{R}^{R_{0}} t^{1-N} \int_{R}^{t} s^{N-1} v_{a}^{q^{*}(s)} d s d t \\
& =v^{*}(R)+\int_{R}^{R_{0}} t^{1-N} \int_{R}^{t} s^{N-1}\left[v_{a}^{q^{*}(s)}-v_{a}^{q_{*}(s)}\right] d s d t+\int_{R}^{R_{0}} t^{1-N} \int_{R}^{t} s^{N-1} v_{a}^{q_{*}(s)} d s d t .
\end{aligned}
$$

Como $0<q(x) \leq \beta$ para $|x| \geq R$, temos $q_{*}(|x|) \leq q(x) \leq q^{*}(|x|) \leq \beta$ e $v_{a}(s) \geq 1$. Fazendo uma conta análoga a (3.18) temos

$$
v_{a}^{q^{*}(s)}-v_{a}^{q_{*}(s)} \leq\left(q^{*}(s)-q_{*}(s)\right) v_{a}^{\beta}(s) \log v_{a}(s)=q_{o s c}(s) v_{a}^{\beta}(s) \log v_{a}(s) .
$$


Assim em (3.28) temos

$$
\begin{aligned}
v\left(R_{0}\right) & \leq v^{*}(R)+\int_{R}^{R_{0}} t^{1-N} \int_{R}^{t} s^{N-1} q_{o s c}(s) v_{a}^{\beta}(s) \log v_{a}(s) d s d t+\int_{R}^{R_{0}} t^{1-N} \int_{R}^{t} s^{N-1} v_{a}^{q_{*}(s)} d s d t \\
& \leq v^{*}(R)+\int_{R}^{R_{0}} t^{1-N} \int_{R}^{t} s^{N-1} q_{o s c}(s) v_{a}^{\beta}(s) \log v_{a}(s) d s d t+\int_{R}^{R_{0}} t^{1-N} \int_{R}^{t} s^{N-1} w_{b}^{q_{*}(s)} d s d t .
\end{aligned}
$$

Como de (3.26) temos $v(s) \leq \varpi(s)$ para $s \geq R$

$\int_{R}^{R_{0}} t^{1-N} \int_{R}^{t} s^{N-1} q_{o s c}(s) v_{a}^{\beta}(s) \log v_{a}(s) d s d t \leq \int_{R}^{R_{0}} t^{1-N} \int_{R}^{t} s^{N-1} q_{o s c}(s) \varpi^{\beta}(s) \log \varpi(s) d s d t$.

Integrando por partes a integral a direita de (3.29)

$$
\begin{aligned}
u & =\int_{R}^{t} s^{N-1} q_{o s c}(s) \varpi^{\beta}(s) \log \varpi(s) d s \quad d u=t^{N-1} q_{o s c}(t) \varpi^{\beta}(t) \log \varpi(t) d t \\
d v & =t^{1-N} d t \quad v=\frac{t^{2-N}}{2-N}
\end{aligned}
$$

temos

$$
\begin{aligned}
\int_{R}^{R_{0}} t^{1-N} \int_{R}^{t} s^{N-1} q_{o s c}(s) \varpi^{\beta}(s) \log \varpi(s) d s d t & =\frac{R_{0}^{2-N}}{2-N} \int_{R}^{R_{0}} s^{N-1} q_{o s c}(s) \varpi^{\beta}(s) \log \varpi(s) d s \\
& -\int_{R}^{R_{0}} \frac{t}{2-N} q_{o s c}(t) \varpi^{\beta}(t) \log \varpi(t) d t \\
& \leq \frac{1}{N-2} \int_{R}^{R_{0}} t q_{\text {osc }}(t) \varpi^{\beta}(t) \log \varpi(t) d t
\end{aligned}
$$

então

$$
\begin{aligned}
v\left(R_{0}\right) & \leq v^{*}(R)+\frac{1}{N-2} \int_{0}^{\infty} t q_{\text {osc }}(t) \varpi^{\beta}(t) \log \varpi(t) d t+\int_{R}^{R_{0}} t^{1-N} \int_{R}^{t} s^{N-1} w_{b}^{q_{*}(s)} d s d t \\
\stackrel{\operatorname{de~} 3.27}{<} & w_{b}(R)+\frac{R w_{b}^{\prime}(R)}{N-2}\left(1-\left(\frac{R}{R_{0}}\right)^{N-2}\right)+\int_{R}^{R_{0}} t^{1-N} \int_{R}^{t} s^{N-1} w_{b}^{q_{*}(s)} d s d t \\
& =w\left(R_{0}\right)
\end{aligned}
$$

assim, temos que $v_{a}\left(R_{0}\right)<w_{b}\left(R_{0}\right)$ contradizendo a definição de $R_{0}$. Portanto $v_{a} \leq w_{b}$ para $r \geq 0$. E desta forma

$$
\triangle v=v(r)^{q^{*}(r)} \geq v(x)^{q(x)} \quad \text { e } \quad \triangle w=w(r)^{q_{*}(r)} \leq w(x)^{q(x)} \text { em } \mathbb{R}^{n}
$$


pois $v(x)=v_{a}(|x|) \geq a \geq 1$ e $w(x)=w_{b}(|x|) \geq v(r) \geq 1$. Portanto $v$ é subsolução do problema (3.1), pelo Teorema 2.10 de [14] existe uma solução $u$ para o problema (3.1) tal que $v \leq u \leq w$ em $\mathbb{R}^{n}$.

Para o caso $\beta=1$ apenas algumas mudanças serão necessárias, mas o passo a passo da prova é o mesmo.

Vamos mostrar primeiramente que a integral

$$
\int_{0}^{\infty} r q_{o s c}(r) \varpi(r) \log \varpi(r) d r
$$

é finita. Para isso, seja $f(x)=x \log x-x^{2 N}$ e

$$
\lim _{x \rightarrow \infty} \frac{\log x}{x^{2 N-1}}=\lim _{x \rightarrow \infty} \frac{\frac{1}{x}}{(2 N-1) x^{2 N-2}}=\lim _{x \rightarrow \infty} \frac{1}{(2 N-1) x^{2 N-1}}=0
$$

então

$$
\lim _{x \rightarrow \infty} f(x)=\lim _{x \rightarrow \infty} x^{2 N}\left(\frac{\log x}{x^{2 N-1}}-1\right)=-\infty
$$

assim, para $N>0$ existe $M>0$ tal que $x>M$ então $f(x)<-N$. Assim para $x$ suficientemente grande $f(x)<0$ e $x \log x<x^{2 N}$. Ou seja, para $r>C$ temos $\varpi(r)>M$ então $\varpi(r) \log \varpi(r)<\varpi(r)^{2 N}$

$$
\int_{0}^{\infty} r q_{o s c}(r) \varpi(r) \log \varpi(r) d r \leq k_{1}+\int_{C}^{\infty} r q_{o s c}(r) \varpi^{2 N}(r) .
$$

Como para o caso $\beta=1$ temos $\varpi(r)=u(R) e^{\left(\frac{R u^{\prime}(R)}{N-2}+\frac{r^{2}}{2 N}\right)}$ então

$$
\begin{aligned}
\int_{0}^{\infty} r q_{o s c}(r) \varpi(r) \log \varpi(r) d r & \leq k_{1}+\int_{C}^{\infty} r q_{o s c}(r) C_{2} e^{\frac{2 N R u^{\prime}(R)}{N-2}} e^{r^{2}} d r \\
& \leq k_{1}+k_{2} \int_{C}^{\infty} r q_{o s c}(r) e^{r^{2}} d r<\infty
\end{aligned}
$$

Assim provamos que a integral (3.31) é finita. Agora podemos tomar $b$ tal que

$$
b>v(R)+\frac{R v^{\prime}(R)}{N-2}+\frac{1}{N-2} \int_{0}^{\infty} r q_{o s c}(r) \varpi(r) \log \varpi(r) d r .
$$

e continuando a prova análogo ao caso $0<\beta<1$ podemos concluir que (3.1) tem uma solução positiva.

Provamos assim que o problema (3.1) tem solução para o caso que $q(|x|) \leq 1$ desde que $|x|$ seja suficientemente grande. 


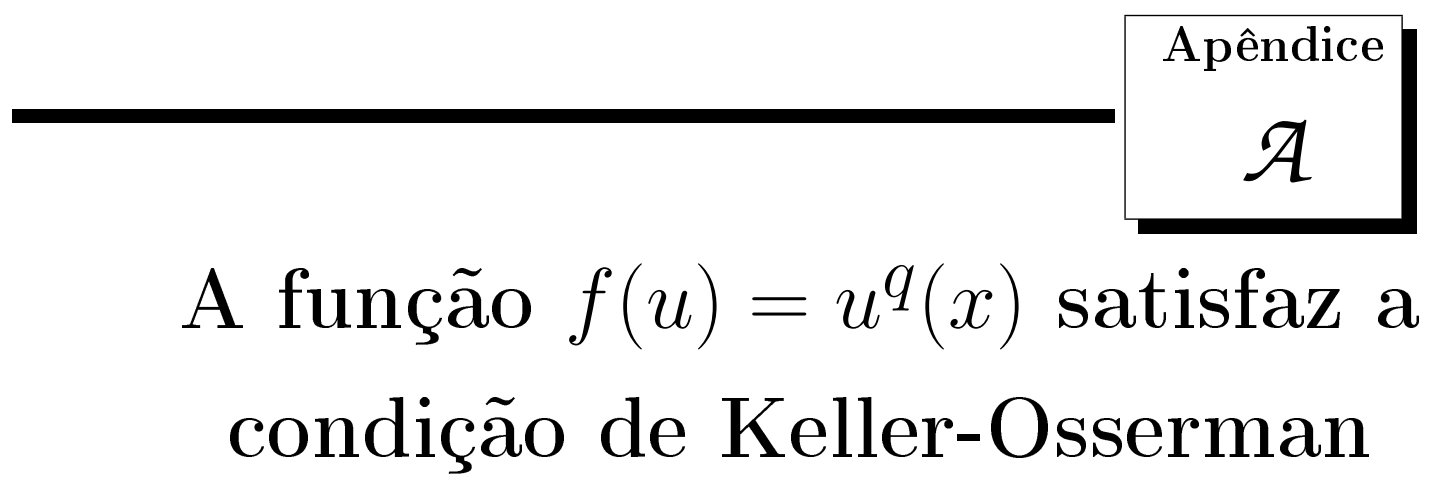

Definição A.1 Seja $f:[0, \infty) \rightarrow[0, \infty)$ é uma função de classe $C^{1}$ não-decrescente tal que $f(0)=0, f(s)>0$ para todo $s>0$ e $f(x)$ satisfaz

$$
\int_{1}^{\infty} \frac{1}{F(t)^{\frac{1}{p}}} d t<\infty \quad \text { onde } \quad F(t):=\int_{0}^{t} f(s) d s
$$

então a função f satisfaz a condição de Keller-Osserman.

A função $f(u)=u^{q}$ é de classe $C^{1}$ em $[0, \infty)$ e é não-decrescente, pois

$$
f^{\prime}(u)=q u^{q-1} \geq 0
$$

e, além disso, $f(0)=0$ e $f(s)=s^{q}>0$ para $s>0$.

Uma função $f:[0, \infty) \rightarrow[0, \infty)$ satisfaz a condição de Keller-Osserman se

$$
\int_{1}^{\infty} \frac{1}{(F(t))^{\frac{1}{p}}} d t<\infty \text { onde } F(t):=\int_{0}^{t} f(s) d s
$$


a função $f(u)=u^{q}$ satisfaz a condição de Keller-Osserman, pois

$$
\begin{aligned}
F(t):=\int_{0}^{t} f(s) d s & =\int_{0}^{t} s^{q} d s=\left.\frac{s^{q+1}}{q+1}\right|_{0} ^{t}=\frac{t^{q+1}}{q+1} \\
\int_{1}^{\infty} \frac{1}{(F(t))^{\frac{1}{p}}} d t & =\int_{1}^{\infty} \frac{(q+1)^{\frac{1}{p}}}{t^{\frac{q+1}{p}}} d t=(q+1)^{\frac{1}{p}} \int_{1}^{\infty} t^{-\frac{q+1}{p}} \\
& =\left.(q+1)^{\frac{1}{p}} \frac{t^{1-\frac{q+1}{p}}}{1-\frac{q+1}{p}}\right|_{1} ^{\infty}=0-\frac{(q+1)^{\frac{1}{p}}}{1-\frac{q+1}{p}}<\infty .
\end{aligned}
$$




\section{Apêndice}

Para mostrar que existe solução para o problema (3.3) vamos usar o Teorema do Ponto Fixo (ver [4]). Para isso, temos que mostrar que o operador

$$
T(u(r))=a+\int_{0}^{r} t^{1-N} \int_{0}^{t} s^{N-1} g(u(s), s) d s d t
$$

é uma contração para $r$ suficientemente pequeno.

Como a função $g$ é contínua, usando aproximação em $C^{\infty}([0, r])$, existe $C>0$ tal que

$$
|g(u(s), s)-g(v(s), s)| \leq C|u(s)-v(s)| .
$$

Então

$$
\begin{aligned}
|T(u(r))-T(v(r))| & \leq \int_{0}^{r} t^{1-N} \int_{0}^{t} s^{N-1}|g(u(s), s)-g(v(s), s)| d s d t \\
& \leq \int_{0}^{r} t^{1-N} t^{N-1} \int_{0}^{t}|g(u(s), s)-g(v(s), s)| d s d t \\
& =\int_{0}^{r} \int_{0}^{t} C|u(s)-v(s)| d s d t \\
& \leq C \int_{0}^{r} \int_{0}^{t}|u-v|_{\infty} d s d t
\end{aligned}
$$




$$
=C_{1}|u-v|_{\infty} r^{2}
$$

para $r \in\left[0, \sqrt{\frac{1}{C_{1}}}\right)$ temos $T(u(r))$ é uma contração, logo pelo Teorema do Ponto Fixo existe $u(r)$ tal que $u(r)=T(u(r))$ assim existe uma solução para a equação (3.3). Portanto

$$
u(r)=a+\int_{0}^{r} t^{1-N} \int_{0}^{t} s^{N-1} g(u(s), s) d s d t
$$

para $r \in\left[0, C_{2}\right)$. 


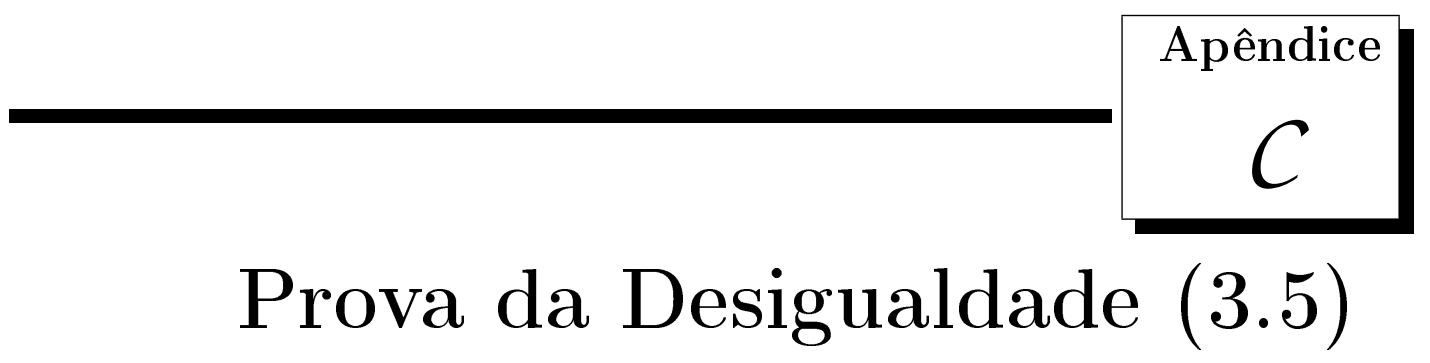

Para facilitar os cálculos no Lema (3.1.2) vamos usar o Teorema de Fubini na integral que segue:

$$
\begin{gathered}
\int_{R}^{r} t^{1-N} \int_{R}^{t} s^{N-1} g(u(s), s) d s d t=\int_{R}^{r} \int_{R}^{t} t^{1-N} s^{N-1} g(u(s), s) d s d t= \\
\int_{R}^{r} \int_{s}^{r} t^{1-N} s^{N-1} g(u(s), s) d t d s=\left.\int_{R}^{r} s^{N-1} g(u(s), s) \frac{t^{2-N}}{2-N}\right|_{s} ^{r} d s= \\
\int_{R}^{r} \frac{r^{2-N}-s^{2-N}}{2-N} s^{N-1} g(u(s), s) d s \leq \int_{R}^{r} s^{2-N} g(u(s), s) s^{N-1} d s= \\
\int_{R}^{r} s g(u(s), s) d s
\end{gathered}
$$

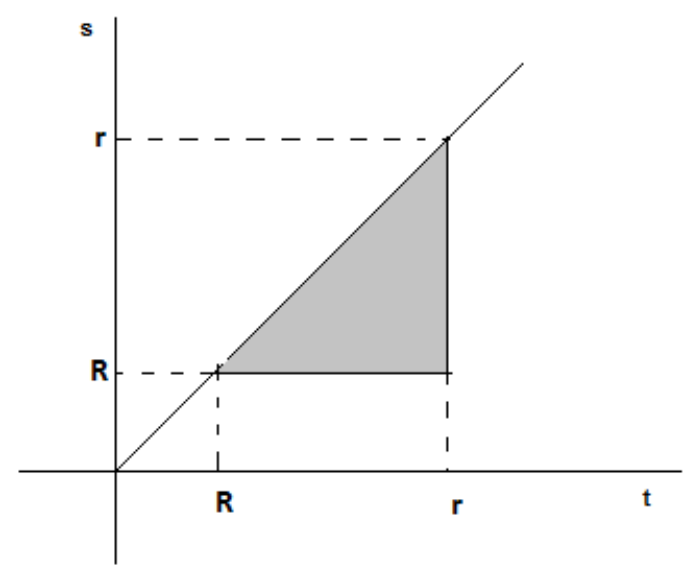




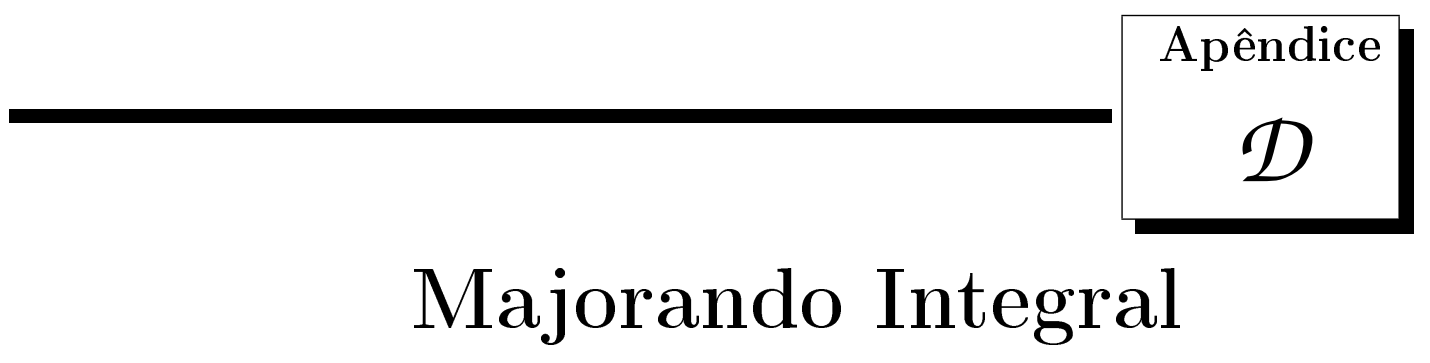

Vamos usar o Teorema de Fubini para majorar a seguinte integral

$$
\begin{aligned}
& \int_{0}^{r} t^{1-N} \int_{0}^{t} s^{N-1}\left|g\left(u_{a}(s), s\right)-g\left(u_{A}(s), s\right)\right| d s d t=\int_{0}^{r} \int_{0}^{t} t^{1-N} s^{N-1}\left|g\left(u_{a}(s), s\right)-g\left(u_{A}(s), s\right)\right| d s d t \\
& =\int_{0}^{r} \int_{s}^{r} t^{1-N} s^{N-1}\left|g\left(u_{a}(s), s\right)-g\left(u_{A}(s), s\right)\right| d t d s=\int_{0}^{r} s^{N-1}\left|g\left(u_{a}(s), s\right)-g\left(u_{A}(s), s\right)\right| \int_{s}^{r} t^{1-N} d t d s \\
& =\int_{0}^{r} s^{N-1}\left|g\left(u_{a}(s), s\right)-g\left(u_{A}(s), s\right)\right| \frac{r^{2-N}-s^{2-N}}{2-N} d s \leq \int_{0}^{r} s^{N-1}\left|g\left(u_{a}(s), s\right)-g\left(u_{A}(s), s\right)\right| s^{2-N} d s \\
& =\int_{0}^{r} s\left|g\left(u_{a}(s), s\right)-g\left(u_{A}(s), s\right)\right| d s .
\end{aligned}
$$






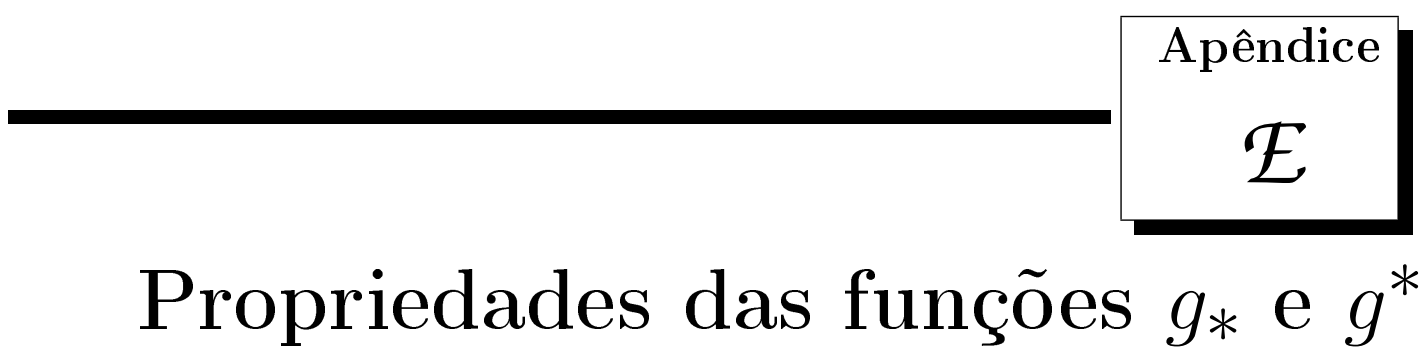

As funções $g_{*}(z, r) g^{*}(z, r)$ definidas na seção (3.2) como

$$
g_{*}(z, r)=\min \left\{z^{q_{*}(r)}, z^{q^{*}(r)}\right\} \quad g^{*}(z, r)=\max \left\{z^{q_{*}(r)}, z^{q^{*}(r)}\right\}
$$

podem ser escritas por

$$
\begin{aligned}
& g_{*}(z, r)=\frac{z^{q_{*}(r)}+z^{q^{*}(r)}}{2}-\frac{\left|z^{q_{*}(r)}-z^{q^{*}(r)}\right|}{2} \\
& g^{*}(z, r)=\frac{z^{q_{*}(r)}+z^{q^{*}(r)}}{2}+\frac{\left|z^{q_{*}(r)}-z^{q^{*}(r)}\right|}{2} .
\end{aligned}
$$

De fato, se $g_{*}(z, r)=z^{q_{*}(r)} \quad \Rightarrow \quad z^{q_{*}(r)} \leq z^{q^{*}(r)}$ então

$$
\frac{z^{q_{*}(r)}+z^{q^{*}(r)}}{2}-\frac{\left|z^{q_{*}(r)}-z^{q^{*}(r)}\right|}{2}=\frac{z^{q_{*}(r)}+z^{q^{*}(r)}+z^{q_{*}(r)}-z^{q^{*}(r)}}{2}=z^{q_{*}(r)}=g_{*}(z, r)
$$

se $g_{*}(z, r)=z^{q^{*}(r)} \quad \Rightarrow \quad z^{q^{*}(r)} \leq z^{q_{*}(r)}$ então

$$
\frac{z^{q_{*}(r)}+z^{q^{*}(r)}}{2}-\frac{\left|z^{q_{*}(r)}-z^{q^{*}(r)}\right|}{2}=\frac{z^{q_{*}(r)}+z^{q^{*}(r)}-z^{q_{*}(r)}+z^{q^{*}(r)}}{2}=z^{q^{*}(r)}=g_{*}(z, r) .
$$

E se $g^{*}(z, r)=z^{q_{*}(r)} \quad \Rightarrow \quad z^{q_{*}(r)} \geq z^{q^{*}(r)}$ então

$$
\frac{z^{q_{*}(r)}+z^{q^{*}(r)}}{2}+\frac{\left|z^{q_{*}(r)}-z^{q^{*}(r)}\right|}{2}=\frac{z^{q_{*}(r)}+z^{q^{*}(r)}+z^{q_{*}(r)}-z^{q^{*}(r)}}{2}=z^{q_{*}(r)}=g^{*}(z, r)
$$


e se $g^{*}(z, r)=z^{q^{*}(r)} \quad \Rightarrow \quad z^{q_{*}(r)} \leq z^{q^{*}(r)}$ então

$$
\frac{z^{q_{*}(r)}+z^{q^{*}(r)}}{2}+\frac{\left|z^{q_{*}(r)}-z^{q^{*}(r)}\right|}{2}=\frac{z^{q_{*}(r)}+z^{q^{*}(r)}-z^{q_{*}(r)}+z^{q^{*}(r)}}{2}=z^{q^{*}(r)}=g^{*}(z, r) .
$$




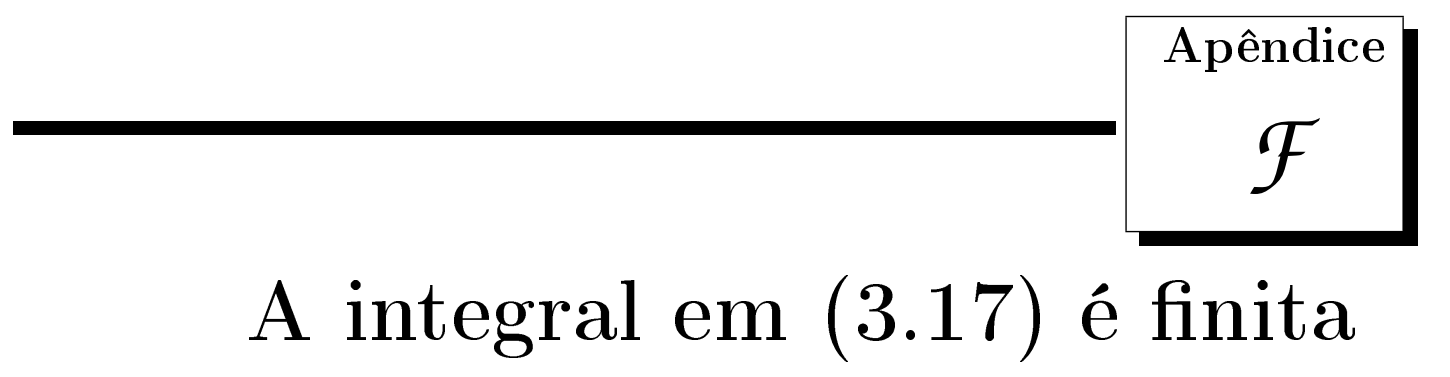

Vamos mostrar que a integral $\int_{0}^{\infty} r q_{o s c}(r) \zeta(r) \log \zeta(r) d r$ é finita. Primeiramente mostraremos que $\zeta(r) \log \zeta(r) \leq \zeta(r)^{2 N \lambda}$. Seja a função $f(x)=x \log x-x^{2 N \lambda}$ então

$$
f^{\prime}(x)=x^{2 N \lambda-1}\left(\frac{\log x}{x^{2 N \lambda-1}}+\frac{1}{x^{2 N \lambda-1}}-2 N \lambda\right)
$$

como

$$
\lim _{x \rightarrow \infty} \frac{\log x}{x^{2 N \lambda-1}}=\lim _{x \rightarrow \infty} \frac{\frac{1}{x}}{(2 N \lambda-1) x^{2 N \lambda-2}}=\lim _{x \rightarrow \infty} \frac{1}{(2 N \lambda-1) x^{2 N \lambda-1}}=0
$$

então usando (F.1) temos

$$
\lim _{x \rightarrow \infty} f(x)=\lim _{x \rightarrow \infty}\left(x \log x-x^{2 N \lambda}\right)=\lim _{x \rightarrow \infty} x^{2 N \lambda}\left(\frac{\log x}{x^{2 N \lambda-1}}-1\right)=-\infty<0
$$

Para $x$ suficientemente grande $f(x)<0$ então $x \log x<x^{2 N \lambda}$ então

$$
\begin{aligned}
\zeta(r) \log \zeta(r) \leq \zeta(r)^{2 N \lambda} & =v(R)^{2 N \lambda} \exp \left(\frac{2 N \lambda R v^{\prime}(R)}{N-2}+\lambda r^{2}\right) \\
& =v(R)^{2 N \lambda} \exp \left(\frac{2 N \lambda R v^{\prime}(R)}{N-2}\right) e^{\lambda r^{2}}=C e^{\lambda r^{2}}
\end{aligned}
$$

assim

$$
\int_{0}^{\infty} r q_{o s c}(r) \zeta(r) \log \zeta(r) d r \leq C \int_{0}^{\infty} r q_{o s c}(r) e^{\lambda r^{2}} d r<\infty
$$




\section{Anexo 1}

Considere o problema

$$
\left\{\begin{array}{l}
\triangle_{p} u=g(x) f(u), \quad x \in D, \\
u(x) \rightarrow \infty, \quad \operatorname{com} d(x, \partial \Omega) \rightarrow 0
\end{array}\right.
$$

vamos estudar a existência de solução $u \in W_{l o c}^{1, p}(D) \cap C(D)$, onde $D \subset \mathbb{R}^{n}$ um domínio limitado, $g$ é uma função não negativa e a função $f$ é uma função não linear que satisfaz:

$f:[0, \infty) \rightarrow[0, \infty)$ é uma função não decrescente $C^{1}$ tal que $f(0)=0$ e $f(s)>0$ para $s>0$

e $f$ satisfaz a condição de Keller-Osserman.

A função $g$ é $C_{\Omega}$-positiva se satisfaz:

"para algum $x_{0} \in D$ satisfazendo $g\left(x_{0}\right)=0$, existe um sub domínio $O \subset D$ contendo $x_{0}$ tal que $g(x)>0 \quad \forall x \in \partial D$."

então o próximo teorema garante existência de solução blow-up.

Lema F.1 Seja $D \subset \mathbb{R}^{n}$ um dominio limitado, e suponha $g \in C(\bar{D})$ e $C_{\Omega}$-positiva em $D$. Se $f$ satisfaz a condição de Keller-Osserman. Então o problema

$$
\left\{\begin{array}{l}
\triangle_{p} u=g(x) f(u), \quad x \in D, \\
u(x) \rightarrow \infty, \quad \operatorname{com} d(x, \partial D) \rightarrow 0
\end{array}\right.
$$

admite uma solução não negativa $u \in W_{l o c}^{1, p}(D) \cap C^{1, \alpha}(D)$ com $0<\alpha<1$.

A demonstração se encontra em [13]. 


\section{Anexo 2}

Lema F.2 Sejam $x, y, \in \mathbb{R}^{n}$ e seja $<,>$ o produto escalar canônico de $\mathbb{R}^{n}$. Então:

$$
<|x|^{p-2} x-|y|^{p-2} y, x-y>\geq\left\{\begin{array}{lll}
c|x-y|^{p} & \text { se } & p \geq 2 \\
c \frac{|x-y|^{2}}{(|x|+|y|)^{2-p}} & \text { se } & 1<p<2
\end{array}\right.
$$

onde $c>0$ é uma constante que depende de $p$.

A prova desse Lema pode ser encontrada em [16] 


\section{Referências Bibliográficas}

[1] R. A. Adams, Sobolev Spaces, Academic Press, (1975).

[2] G. Díaz, R. Letelier, Explosive solutions of quasilinear elliptic equations: Existence and uniqueness, Nonlinear Analysis, 20 (1993), 97-125.

[3] E. DiBenedetto, $C^{1+\alpha}$ local regularity of weak solutions of degenerate elliptic equations, Nonlinear Analysis, 7 (1983), 827-850.

[4] L. C. Evans, Partial Differential Equations, Graduate Studies in Mathematics, Volume 19, American Mathematical Society, (1997).

[5] J. García-Melián, Large solutions for equations involving the p-Laplacian and singular weights, Zeitschrift für angewandte Mathematik und Physik, 60 (2009), 594-607.

[6] J. García-Melián, J. C. Sabina de Lis, Large solutions for the Laplacian with a power nonlinearity given by a variable exponent, Annales de l'Institut Henri Poincaré Analyse Non Linéaire, 26 (2009), 889-902.

[7] D. Gilbarg, N. S. Trudinger, Elliptic Partial Differential Equations of Second Order, Springer, (1998).

[8] T. Kura, The weak supersolution-subsolution method for second order quasilinear elliptic equations, Hiroshima Mathematical Journal 19 (1989), 1-36.

[9] A. V. Lair, A. Mohammed, Entire Large Solutions to Elliptic Equations of Power Non-linearities with Variable Exponents, Advanced Nonlinear Studies 13 (2013), 699-719.

[10] A. V. Lair, A. W. Wood, Large solutions of semilinear elliptic problems, Nonlinear Analysis, 37 (1999), 805-812. 
[11] A. T. Lourêdo, Minicurso Introdução aos métodos Variacionais, Universidade Estadual da Paraíba, (2014).

[12] L. S. Martin, Notas de aulas de Equação Diferncial Ordinária, UNICAMP.

[13] A. Mohammed, Existence and asymptotic behavior of blow-up solutions to weighted quasilinear equations, Journal of Mathematical Analysis and Applications 298 (2004), $621-637$.

[14] W. M. Ni, On the elliptic equation $\triangle u+K(x) u^{\frac{(n+2)}{n-2}}=0$, its generalizations, and applications in geometry, Indiana University Mathematics Journal, $31 \mathrm{n}^{\circ} 04$ (1982), $493-529$.

[15] R. Osserman, On the inequality $\triangle u \geq f(u)$, Pacific Journal of Mathematics, 7 (1957), $1641-1647$.

[16] I. Peral, Métodos Variacionales y Ecuaciones em Derivadas Parciales.

[17] J. Sotomayor, Lições de Equações Diferenciais Ordinárias, Projeto Euclides IMPA, (1942).

[18] P. Tolksdorf, On the Dirichlet problem for quasilinear equations in domains with conical boundary points, Comm. in Partial Differential Equations 8 (1983), 773-817.

[19] J. L Vázquez, A Strong Maximum Principle for Some Quasilinear Elliptic Equations, Applied Mathematics and Optimization 12 (1984), 191-202.

[20] Z. Zhang, A remark on the existence of explosive solutions for a class of semilinear elliptic equations, Nonlinear Analysis, 41 (2000), 143-148. 